\title{
OPTIMIZTTIC
}

\section{OPTIMIZing the Treatment of}

\section{Trauma Induced Coagulopathy}

\author{
Cand Med Kjersti Baksaas-Aasen \\ Faculty of Medicine, University of Oslo \\ Department of Traumatology, Oslo University Hospital \\ Department of Anaesthesiology, Oslo University Hospital
}


(C) Kjersti Baksaas-Aasen, 2021

Series of dissertations submitted to the Faculty of Medicine, University of Oslo

ISBN 978-82-8377-921-9

All rights reserved. No part of this publication may be reproduced or transmitted, in any form or by any means, without permission.

Cover: Hanne Baadsgaard Utigard.

Print production: Reprosentralen, University of Oslo. 


\section{TABLE OF CONTENT}

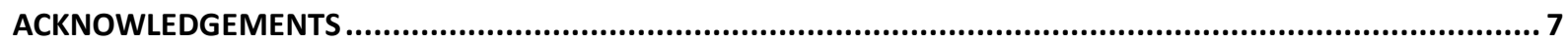

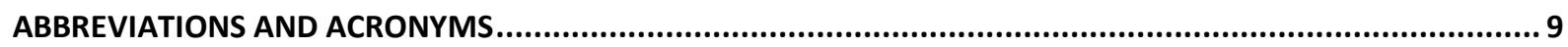

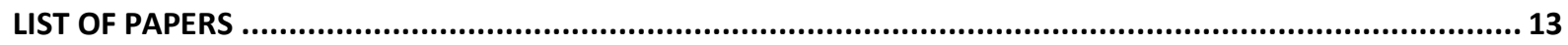

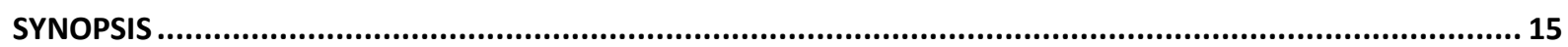

INTRODUCTION

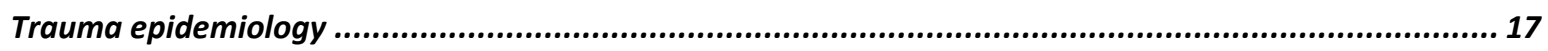

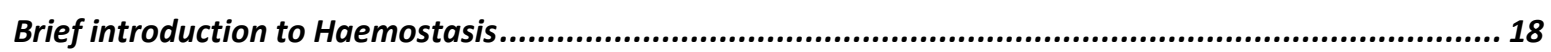

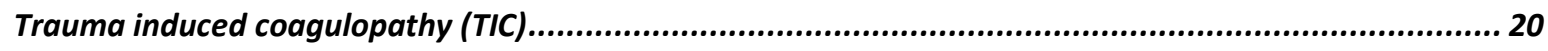

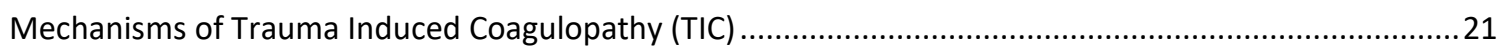

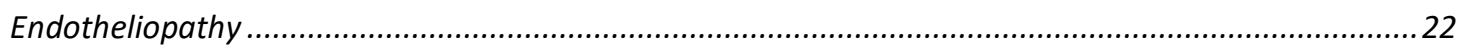

Protein C

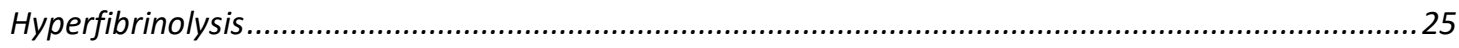

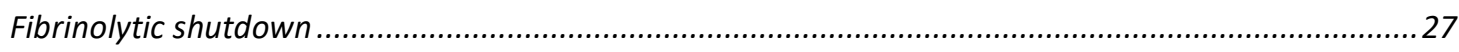

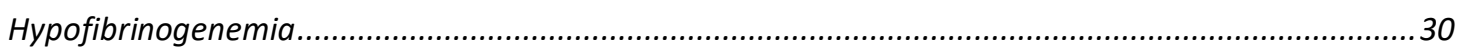

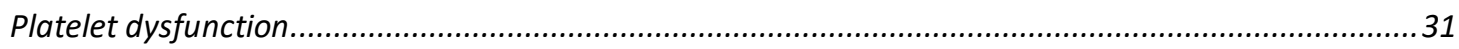

Classical trauma triad - hypothermia, haemodilution and acidaemia................................................... 32

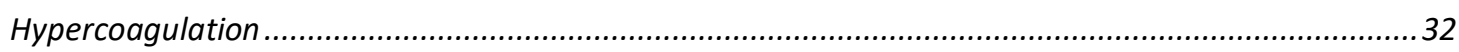

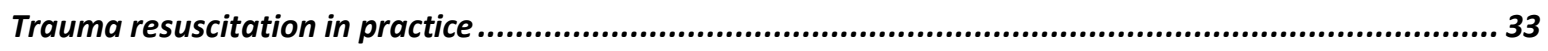

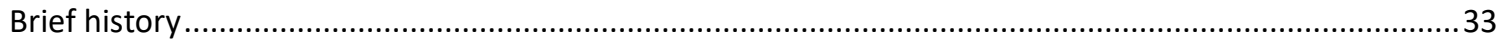

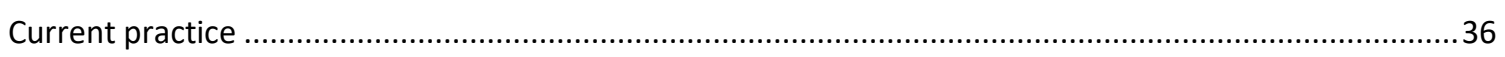

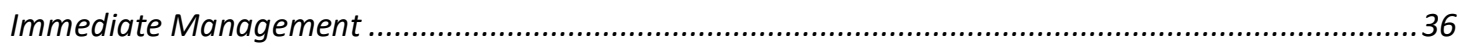

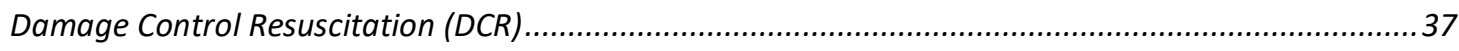

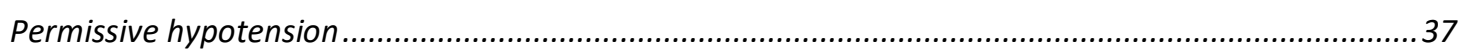

Massive Transfusion Protocol (MTP) and haemostatic resuscitation ..................................................39 


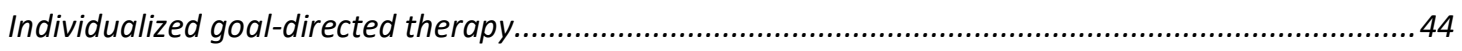

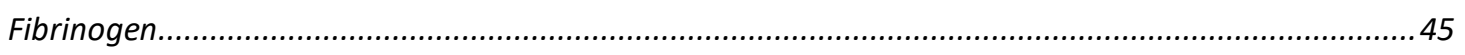

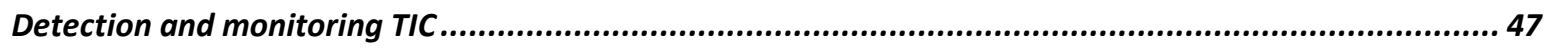

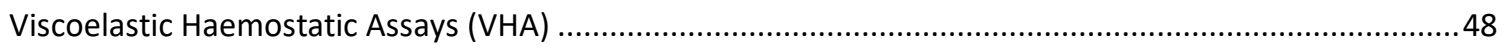

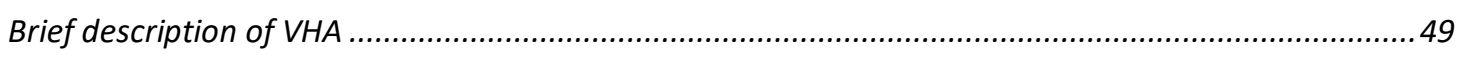

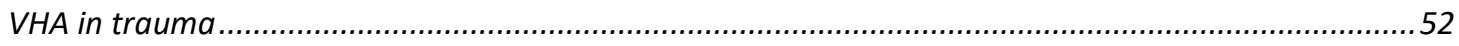

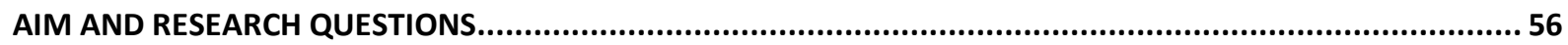

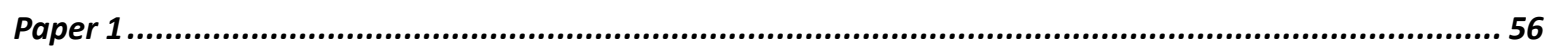

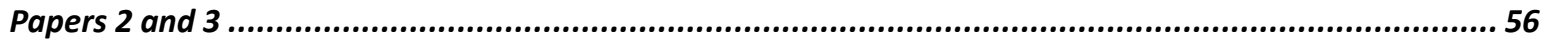

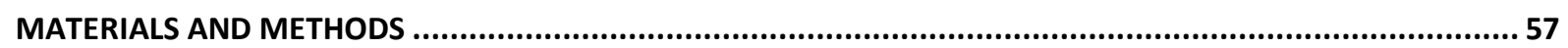

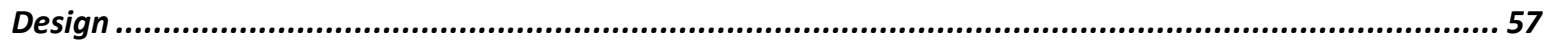

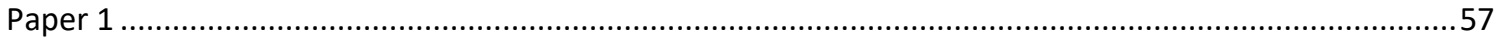

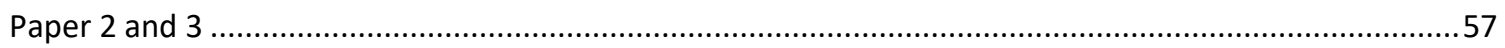

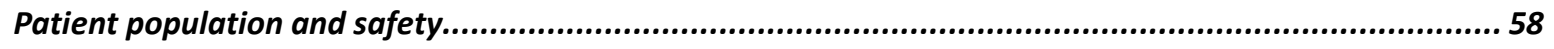

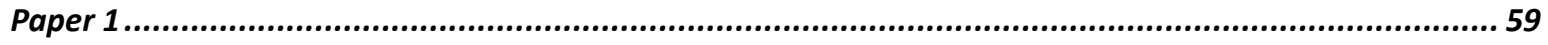

Data collection, sampling techniques and measurements for development of algorithms ......................59

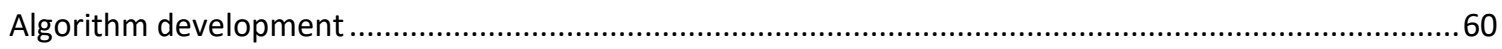

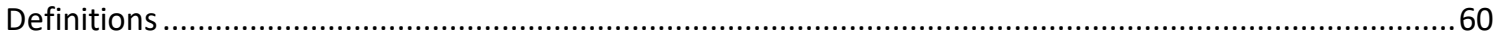

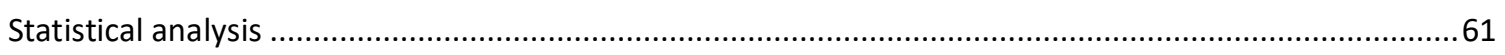

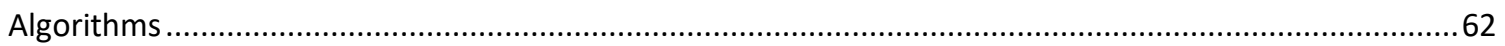

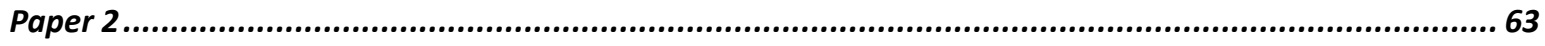

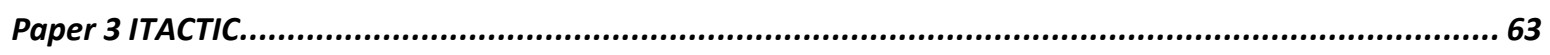

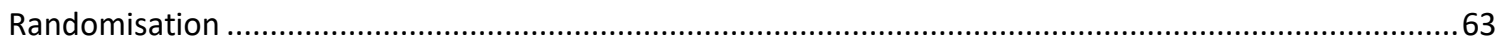

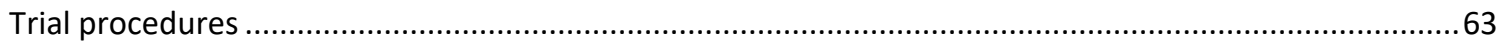

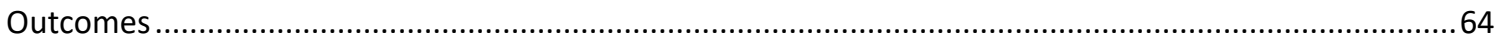


RESULTS

Paper 1. 66

Paper 3 ITACTIC

DISCUSSION 71

Trauma resuscitation algorithms . 71

VHA vs CCTs. 72

TXA, fibrinolysis and brain injury. 75

Best practice management of trauma haemorrhage in the future ............................................ 80

The future of trauma resuscitation.......................................................................................81

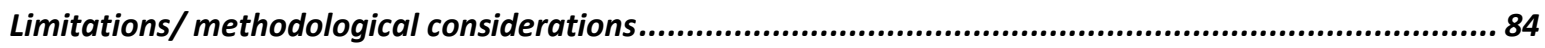

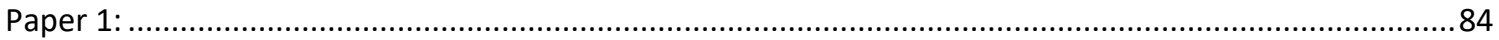

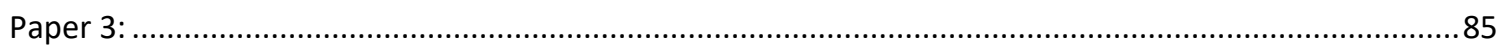

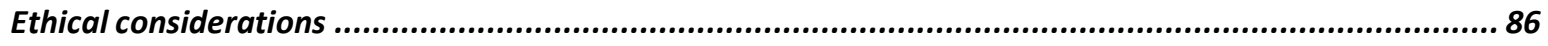

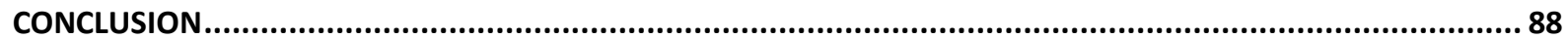

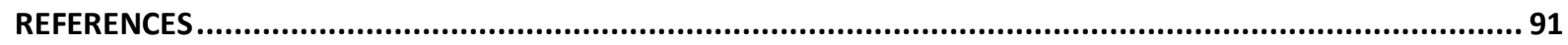




\section{ACKNOWLEDGEMENTS}

First and foremost, I want to thank my supervisor, friend, and head of Department of Traumatology Tina Gaarder for giving me the possibility to be a part of this research project. $\mathrm{My} \mathrm{PhD}$ had never been possible without you and your international network. Equal thanks to my co-supervisor Pål Aksel Næss. You are both extremely dedicated, have an enormous working capacity and extensive knowledge and experience in trauma. I am grateful for being part of your team.

My thesis is based on data from the trauma centres in the TACTIC collaboration; Oslo University Hospital, The Royal London Hospital (London, UK), John Radcliffe Hospital (Oxford, UK), Academic Medical Centre (Amsterdam, Netherlands), Rigshospitalet (Copenhagen, Denmark) and Kliniken der Stadt Köln gGmbH (Cologne, Germany). I am humble by the possibility to be part of the TACTIC collaboration, and for me the opportunity to do a PhD within the TACTIC project has been an extremely instructive and truly inspiring journey. Despite some frustrating moments, I would not have been without it. Many thanks to all the highly knowledgeable and enthusiastic colleagues in the TACTIC collaboration, especially Karim Brohi, Nicole Juffermans and Per I Johansson for their support and critical advice in my $\mathrm{PhD}$ project.

Many thanks also to all trauma coordinators in the Department of Traumatology. The TACTIC project has been completely dependent on the work of the trauma coordinators with continuously collecting data, running VHA tests and following up every patient 24-7.

I am also very grateful for the friendship, flexibility and support granted by the leaders of the Department of Anaesthesiology, and especially Anne Bøen. Without their flexibility and endless support, my shared position between two different departments would have been significantly more demanding.

I will also thank all my colleagues in the Department of Traumatology and Department of Anaesthesiology, Ullevål. I really appreciate the social environment in both departments with an extremely friendly atmospheres and good discussions around clinical work, science or just 
pleasure. Special thanks to my anaesthesiologic partner in the Department of Traumatology Anders Holtan for always be available when I need to discuss whatever.

And last, but not least, I will thank my family for constantly and unconditional support. 


\section{ABBREVIATIONS AND ACRONYMS}

A

a2AP

ACIT

ACS

ACT

aPC

aPTT

ARDS

ATC

ATIII

ATLS

AUC

BD

$\mathrm{BE}$

CA

CCT

CFT

CL

$\mathrm{CO}_{2}$

CRF

CT

DCR

DCS
Amplitude (TEG)

Alpha-2-antiplasmin

Activation of Coagulation and Inflammation in Trauma

Abdominal compartment syndrome

Activated clotting time (TEG)

Activated Protein C

Activated partial Thromboplastin time

Acute respiratory distress syndrome

Acute traumatic coagulopathy

Antithrombin III

Advanced Trauma Life Support

Area under curve

Base deficit

Base excess

Clot amplitude

Conventional coagulation test

Clot formation time

Clot lysis (ROTEM)

Carbon dioxide

Case report form

Clotting time

Damage control resuscitation

Damaged controlled surgery 
Disseminated intravascular coagulation

ROTEM assay, activated by tissue factor

FF TEG

TEG assay, Functional Fibrinogen TEG,

activated by tissue factor and platelets inhibited by Abciximab

FFP

Fresh frozen plasma

FIBTEM

ROTEM assay,

activated by tissue factor and platelets are inhibited by cytochalasin D

GCS

Glascow coma scale

HIV

Human immunodeficiency viruses

ICU

Intensive care unit

INTEM

ROTEM assay, contact activated

INR

International normalized ratio

INTRN

International Trauma Research Network

IQR

Interquartile range

ISS

Injury severity score

ITACTIC

Implementing Treatment Algorithms for the Correction of

Trauma-induced Coagulopathy

ITT

Intention to treat

K

Kinetics (used as K-time in TEG)

$\mathrm{Li}$

Lysis index (ROTEM)

LI30

Lysis index at 30 minutes (ROTEM) 
Massive transfusion protocol

Odds ratio

Plasmin activator inhibitor 1

PAP

Plasmin-antiplasmin

PC

Protein C

POC

Point of care

PP

Per protocol

PROMMT

Prospective, observational, multicentre, major trauma transfusion study

PROPPR

The pragmatic, randomized optimal platelet and plasma ratios trial

PT

Prothrombin time

$\mathrm{PTr}$

Prothrombin time ratio

$\mathrm{R}$

Reaction (used as R-time in TEG)

$\mathrm{RBC}$

Red blood cell

RCT

Randomized controlled trial

ROTEM

Rotational thromboelastometry (brand name)

ROC

Reciever operating characteristics

rTEG 
SHINE

TACTIC

TAFI

TBI

TEG

$\mathrm{TF}$

TFPI

TIC

TM

tPA

TXA

$\mathrm{uPa}$

VHA
Shock-induced endotheliopathy

Targeted Action for Curing Trauma Induced Coagulopathy

Thrombin activatable fibrinolysis inhibitor

Traumatic brain injury

Thromboelastography (brand name)

Tissue factor

Tissue factor pathway inhibitor

Trauma induced coagulopathy

Thrombomodulin

Tissue-type plasminogen activator

Tranexamic acid

Urinary-type plasminogen activator

Viscoelastic Haemostatic Assay 


\section{LIST OF PAPERS}

\section{Paper 1}

Data-driven development of ROTEM and TEG algorithms for the management of trauma haemorrhage - a prospective observational multicentre study [1].

Baksaas-Aasen K, va Dieren S, Balvers K, Juffermans N, Næss PA, Rourke C, Eaglestone S, Ostrowski SR, Stensballe J, Stanworth S, Maegele M, Goslings JC, Johansson PI, Brohi K, Gaarder C, TACTIC/INTRN collaborators (2019) Data-driven Development of ROTEM and TEG Algorithms for the Management of Trauma Hemorrhage. Ann Surg. 2019

Dec;270(6):1178-1185. doi: 10.1097/SLA.0000000000002825.

\section{https://dx.doi.org/10.1097/sla.0000000000002825}

\section{Paper 2}

iTACTIC - implementing Treatment Algorithms for the Correction of Trauma-

Induced Coagulopathy: study protocol for a multicentre, randomised controlled trial [2].

Baksaas-Aasen K, Gall L, Eaglestone S, Rourke C, Juffermans NP, Goslings JC, Naess PA, van Dieren S, Ostrowski SR, Stensballe J, Maegele M, Stanworth SJ, Gaarder C, Brohi, K, Johansson PI (2017) iTACTIC - implementing Treatment Algorithms for the Correction of Trauma-Induced Coagulopathy: study protocol for a multicentre, randomised controlled trial. Trials. 2017 Oct 18;18(1):486. doi: 10.1186/s13063-017-2224-9.

https://dx.doi.org/10.1186/s13063-017-2224-9 


\section{Paper 3}

Viscoelastic haemostatic assay augmented protocols for major trauma haemorrhage (ITACTIC): a randomised, controlled trial [3].

Baksaas-Aasen K, Gall LS, Stensballe J, Juffermans NP, Curry N, Maegele M, Brooks A, Rourke C, Gillespie S, Murphy J, Maroni R, Vulliamy P, Henriksen HH, Pedersen KH, Kolstadbraaten KM, Wirtz MR, Kleinveld DJB, Schäfer N, Chinna S, Davenport RA, Naess PA, Goslings JC, Eaglestone S, Stanworth S, Johansson PI, Gaarder C, Brohi K (2021) Viscoelastic haemostatic assay augmented protocols for major trauma haemorrhage (ITACTIC): a randomized, controlled trial. Intensive Care Med. 2021 Jan;47(1):49-59. doi: 10.1007/s00134-020-06266-1. Epub 2020 Oct 13.

https://dx.doi.org/10.1007/s00134-020-06266-1 


\section{SYNOPSIS}

Injuries account for approximately $9 \%$ of the global mortality, and traumatic brain injury and major haemorrhage appear to be the two major causes of trauma deaths.

As a result of better understanding of the pathophysiology of trauma induced bleeding and improved resuscitation-strategies, the mortality from major haemorrhage has decreased over the last decades but is still the leading cause of preventable deaths.

Our haemostatic system is activated as a response to bleeding to minimize blood loss. However, in some of the most severely injured patients an endogenous coagulopathy may occur. This endogenous coagulopathy leads to increased mortality and blood product requirement and is named Trauma Induced Coagulopathy (TIC). Targeting TIC has been one of the most important factors in the improvement of trauma resuscitation, and TIC is still a major area of research to further reduce trauma mortality.

Best practice trauma resuscitation includes individualization of treatment. Early detection of patients developing TIC might improve haemostatic therapy and reduce mortality from major haemorrhage and blood product requirement. However, the complete understanding of TIC is still lacking, and there is no ideal test to detect TIC. Hence, the interest for Viscoelastic Haemostatic Assays (VHAs) in trauma resuscitation has increased during the last decade, and VHAs have been widely implemented in trauma centres worldwide. VHAs monitor the coagulation dynamically and clinically relevant parameters can be obtained within 10 minutes, to guide haemostatic therapy. However, VHAs have not been proven superior to conventional coagulation tests (CCTs) in trauma, and there is no universal agreement on which parameters or threshold values to use in trauma resuscitation.

We aimed to produce data-driven algorithms for guiding trauma resuscitation. Moreover, these algorithms were implemented in an international multicentre randomized controlled trial 
(RCT) comparing VHAs versus CCTs in guiding haemostatic therapy to bleeding trauma patients.

The algorithms were constructed based on data from a large cohort of trauma patients prospectively collected in an international observational multicentre study. Clinically useful VHA and CCT parameters and threshold values to identify coagulopathy in haemorrhaging trauma patients were detected. These parameters and threshold values were used to guide individualized haemostatic therapy added to a best practice empiric massive transfusion protocol (MTP).

The algorithms were tested in an investigator-initiated, pragmatic, multi-centred, superiority, parallel-group, RCT performed at 7 major trauma centres in Europe, named ITACTIC (Implementing Treatment Algorithms for the Correction of Trauma-induced Coagulopathy). The aim of this study was to compare VHAs versus CCTs in guiding goal-directed individualized resuscitation in trauma haemorrhage.

ITACTIC showed overall no difference between groups for the primary endpoint alive and free of massive transfusion at 24 hours or any of the secondary endpoints. However, the study showed a reduced mortality in the severe traumatic brain injury (TBI) subgroup for the VHA arm and a non-significant trend towards lower mortality in the coagulopathic subgroup. In addition, study interventions were given significantly earlier and more frequently in the VHA arm. Hence, the results from ITACTIC indicate that for most of the trauma patients, who are not coagulopathic, it does not really matter how you monitor resuscitation, as long as the resuscitation strategy adheres to best existing evidence, the coagulation is monitored, and the protocol is followed. However, when patients are severely injured, coagulopathic and/or with combined bleeding and severe TBI, closer monitoring of the coagulation with rapidly available results from coagulation tests with VHAs might impact survival. This has to be explored further. 


\section{INTRODUCTION}

\section{Trauma epidemiology}

More than 5 million people die each year following injuries [4] which accounts for $9 \%$ of the global mortality. Approximately a quarter of all trauma deaths are the result of suicide and homicide, while road traffic injuries account for nearly another quarter of the deaths. Road accidents are the worldwide leading cause of death for young people 15-29 years old. Other main causes of death from injuries are falls, drowning, burns, poisoning and war [4]. In Norway approximately 2000 people died from injuries in 2018, accounting for about $5 \%$ of all deaths [5].

Traumatic brain injuries and massive haemorrhage appear to be the two major causes of trauma deaths worldwide, and massive haemorrhage is the leading cause of the preventable deaths [6,7]. Following innovation in management of major haemorrhage and better understanding of the trauma physiology, there has been a large reduction in mortality for severely injured trauma patients over the last 10 years [8]. Nevertheless, mortality for the most critically injured patients requiring massive transfusion defined as more than 10 units of red blood cells (RBCs) within 24 hours post injury, hasn't changed much, and still remains close to $50 \%[8,9,10]$.

Most of the haemorrhagic deaths occur prehospitally. Mortality from exsanguination has decreased as a result of trauma systems and damage control interventions including haemostatic resuscitation $[6,11,12]$. As a result, more severely injured and physiologically compromised patients survive to hospital. This has resulted in increased early mortality in hospital (within 3 hours) [8,11]. However, mortality between 3 and 24 hours has been markedly reduced over the last decade, following improvement in trauma resuscitation [8]. 
Nevertheless, the death rate after 24 hours remains high in severely injured patients. These late deaths are split evenly between traumatic brain injury (TBI) and multiple organ failure (MOF) [8], with mortality from TBI remaining consistent over time [12,13]. Many of the TBI patients survive initially, but then die over the following days. On the other hand, the entity of MOF has changed. Historically deaths related to MOF were caused by acute respiratory distress syndrome (ARDS), renal failure or abdominal compartment syndrome (ACS). Today, the pulmonary and renal failures are taken care of by organ support and improved intensive care, and ACS has almost disappeared with improved resuscitation [11]. MOF today is broadly divided into two: the early cardiac and vascular failure resulting in a cardiogenic, hypovolemic and distributive shock, typically developing within hours after successful resuscitation and almost unresponsive to treatment, or a later MOF following persistent inflammation, immunosuppression or multiple episodes of sepsis $[8,11]$.

\section{Brief introduction to Haemostasis}

The haemostatic system manages a process of clot formation following haemorrhage and vascular injury, to maintain sufficient blood pressure and oxygen perfusion to tissues and organs.

When the vessel wall is breached or the endothelium disrupted, collagen and tissue factor (TF) become exposed to the flowing blood, and thereby initiates the formation of a thrombus. Exposed collagen triggers the accumulation and activation of platelets, and exposed TF initiates the generation of thrombin, which activate platelets and converts fibrinogen to fibrin [14].

Historically, the haemostatic system was described as two parallel intrinsic and extrinsic pathways. These pathways consist of proteolytic cascades ending in a common pathway which generates the thrombus. In 2001, the cell-based model was introduced describing three 
different stages of haemostasis: initiation, amplification and propagation [15]. The cell-based theory is believed to better describe the in vivo haemostasis than the cascade model by describing the contribution of the different cells in the haemostasis.

In this theory [15], the coagulation process is controlled by cellular components presented on the surface of the cells involved in the coagulation process. The primary activator of the haemostasis is TF presented by extravascular TF-bearing cells expressed to plasma after a break in the vessel wall. Initiation occurs as Factor VII in plasma binds to cellular TF and the complex of activated Factor VII/TF activates Factor X. Factor X is inhibited if it leaves the cell surface, but Factor $\mathrm{X}$ on the surface of the TF-bearing cell will combine with Factor $\mathrm{V}$ to produce small amounts of thrombin. This thrombin plays an important role in the following amplification phase. Damage to the vasculature also allows platelets to come into contact with extravascular tissues. Platelets adhere to the extravascular matrix components at the site of injury and this process activates the platelets and localizes them near a site of TF exposure. The small amount of thrombin generated on the TF-bearing cells in the initiation phase amplifies the initial procoagulant signal by enhancing platelet adhesion and activates platelets and coagulation factors. During the propagation phase coagulation factor complexes are assembled on the platelet surface and a large-scale thrombin generation takes place. This thrombin burst converts fibrinogen to fibrin, which is the major component of the clot. In the normal state, there is a fine balance between clot formation and breakdown through the anticoagulant, prothrombotic and fibrinolytic pathways. The haemostatic response is counterbalanced by natural endogenous anticoagulants that prevent spontaneous clotting and ensure the response to be proportional to the entity of tissue damage [16]. Thrombin also plays a role in activating anticoagulant factors to prevent runaway coagulation. An important step in this anticoagulant counterbalance is the thrombin-activated protein $\mathrm{C}$ (aPC) pathway [15]. Thrombin escaping into the circulation from an injury site must be controlled to prevent 
clotting in the systemic circulation. This takes place either by inhibition in the plasma by antithrombin III (ATIII) or tissue factor pathway inhibitor (TFPI) or by thrombin binding to thrombomodulin (TM) on intact endothelial cells. TM is a receptor for thrombin and is expressed at high levels on endothelial cells, especially in the microvasculature. The thrombin-TM complex activates Protein C (PC) on the endothelial surface. PC is localized on endothelial surfaces by an endothelial Protein $\mathrm{C}$ receptor (EPCR), that facilitates its activation by thrombin/TM. APC forms a complex with Protein $\mathrm{S}$ that cleaves and inactivates Factor Va and Factor VIIIa activated on endothelial cell surfaces. This prevents the formation of additional procoagulant enzymes at sites where a healthy, intact endothelial lining layer is present. Thus, thrombin is effectively changed from a pro-coagulant to an anti-thrombotic enzyme when it is localized on an endothelial cell surface that expresses TM. In addition to TM, endothelial cells also express other important anti-thrombotic surface features and under normal conditions vascular endothelial cells are highly specialized to be actively antithrombotic. Injury or inflammation can induce endothelial cells to decrease expression of TM and increase expression of TF and other surface adhesion molecules.

In the end, the optimal clot depends on adequate function of each step in the cell-based model for coagulation; amount, time and site of thrombin generation; amount and quality of the fibrin clot; number and function of platelets; integrity of vessels walls and blood flow [16]. When any of these conditions are not met, coagulopathy can take over.

\section{Trauma induced coagulopathy (TIC)}

Prior to the 21 st century, the coagulation of trauma patients was not viewed as a critical driver of patient physiology in trauma. Rather, coagulopathy in injured patients was considered to be iatrogenic, caused by consumption, loss and dilution of coagulation factors following large 
volumes of RBCs and crystalloids and further exacerbated by hypothermia and acidosis $[17,18,19]$.

This triad of coagulopathy, hypothermia and acidosis was defined as a the "triad of death", "the lethal triad" or "the bloody vicious cycle" and was associated with poor outcome [20]. In 2003, the traditional understanding of coagulopathy of trauma as iatrogenic, caused by resuscitation, was disrupted by two simultaneously published, but independent, studies $[21,22]$. These studies described an early coagulopathy defined by increased Prothrombin time (PT) and activated partial thromboplastin time (aPTT) in 25 to $30 \%$ of severely injured patients on admission. This endogenous coagulopathy, independent of resuscitation, increased with injury severity and predicted significantly higher mortality. As a consequence of these studies, trauma resuscitation research shifted towards characterising this endogenous coagulopathy and treatment directed towards correction of it [23]. In the following years, different trauma research networks around the world focused on potential mechanisms involved, and since then numerous of reviews of the pathophysiology of this coagulopathy have been published $[16,17,24,25,26,27,28,29,30,31,32,33,34]$. However, to date the complete understanding of the mechanism behind TIC is still lacking.

\section{Mechanisms of Trauma Induced Coagulopathy (TIC)}

Since 2003 the nomenclature of the coagulopathy developing after injury has varied, but most commonly today this coagulopathy is named Trauma induced Coagulopathy (TIC), consisting of an endogenous part, the Acute Traumatic Coagulopathy (ATC) and the traditionally resuscitation related triad consisting of acidosis, hypothermia and haemodilution $[17,27,35]$.

Since ATC was first described, a variety of theories have attempted to elucidate the biochemical mechanisms [33]. Many of these theories have been looking at only small parts 
of the coagulation system. Lack of precise tests, discrepancies between various tests and devices, no pre-injury measurements, vulnerable patients, critical treatments depending on timely test results and lack of laboratory tests that facilitate an understanding of coagulation as a dynamic process, are some of the reasons for the many, often incomplete and conflicting conclusions presented [33]. There is a relatively universal agreement that ATC is a multifactorial failure of the coagulation system to sustain adequate haemostasis following major trauma. ATC seems to be driven by tissue injury, worsened by a combination of endothelial hypoperfusion and high levels of catecholamines, and is mediated by endothelial dysfunction and glycocalyx degradation, with hyperfibrinolysis, hypocoagulation and early fibrinogen depletion as key components $[17,34,36,37,38]$. Clinically, severely injured patients present a diverse spectrum of phenotypes of ATC varying from hypocoagulability to hypercoagulability, and they may rapidly change between phenotypes $[32,39]$.

\section{Endotheliopathy}

The collective process of endothelial injury, inflammation, vascular leak, glycocalyx degrading and overall endothelial dysfunction after trauma has been named the "endotheliopathy" of trauma [40]. This endotheliopathy is mediated by hypoperfusion, hypoxia, catecholamines and inflammation $[31,41]$.

Johansson and colleagues demonstrated in 2010 how sympathoadrenal activation following trauma leads to a dose dependent surge in circulating catecholamines. Hypercoagulability was demonstrated after moderate catecholamine released followed by hypocoagulation and further hyperfibrinolysis with increasing levels of catecholamines [42]. High concentrations of catecholamines directly damaging the endothelium is important for balancing haemostasis. The glycocalyx is a heterogeneous group of proteoglycan core proteins and glycosaminoglycan chains linked to the luminal side of the vascular endothelium and 
protecting the endothelium to maintain a vascular barrier function. The glycocalyx contains significant amounts of heparin-like substances being released into the circulation upon disruption leading to anticoagulation $[42,43,44,45]$. Degradation of the glycocalyx is suggested to be one of the important contributors to the coagulopathy and hyperfibrinolysis of trauma [44]. The critical role of sympathoadrenal activation as a driver for endothelial glycocalyx and cell damage (endotheliopathy) was reproduced in a larger prospective observational study of more than 400 trauma patients in 2017 [37].

In 2017 Johansson and colleagues also presented a potential unifying pathological link between sympathoadrenal hyperactivation, endotheliopathy and poor outcome in different groups of acute sick patients. The group had studied more than 3000 critically ill patients from severe trauma, sepsis, myocardial infarction and post cardiac arrest syndrome. Features driven by shock were discovered to be shared across the patient populations, and the body's response to the various life-threatening conditions was homogeneous. This relatively uniform endothelial damage was suggested as a new disease entity called shock-induced endotheliopathy (SHINE) [46]. The clinical impact of this entity remains unclear.

\section{$\underline{\text { Protein C }}$}

Endotheliopathy following injury contributes to increased inflammation, tissue and endothelial damage, activation of the PC pathway and release of Weidel-Palade bodies containing tissue-type plasminogen activator (tPA) $[41,44]$. The exact pathophysiology and interplay between these disorders of the coagulation remains a subject of ongoing research [32]. However, the aPC pathway was one of the first described mechanisms behind ATC, presented in 2007 by Brohi [36]. Multiple prospective clinical studies, in-vitro studies and animal models have further supported the aPC pathway as an important mechanism behind 
ATC $[47,48,49,50,51,52]$, and elevated aPC levels have been associated with poor outcome after major trauma $[21,36,47,48]$.

Damage to the endothelium upregulates TM and the EPCR expression on hypoperfused endothelium. Combined with massive thrombin generation following tissue trauma, this drives a thrombin-TM complex formation and accelerate activation of $\mathrm{PC}[30]$. aPC has a systemic anticoagulant effect by inactivating coagulation Factor Va and VIIIa, and in addition consumes Plasminogen Activator Inhibitor-1 (PAI-1). PAI-1 is the major antagonist of tPA, and by consuming the inhibitor of tPA, aPC accelerate clot lysis [30,48] (described below). Davenport and colleagues presented in 2017 a study of 300 trauma patients looking at the mechanisms of ATC [48]. They demonstrated elevated aPC-levels in shocked patients (defined as base deficit (BD) above 6) and high aPC was associated with low PC. Reduced Factor Va and VIIIa activity was also demonstrated with increasing aPC levels. However, this reduction was not associated with any significant increase in clotting time (CT) on Rotational thromboelastometry (ROTEM) or extensive prolongation of Prothrombin time ratio (PTr), which suggests that systemic aPC-induced anticoagulation is not the primary mechanism of ATC. Levels of other procoagulant factors not targeted by aPC and thrombin generation were preserved in the patients with ATC, suggesting that consumption of procoagulant factors and reduced thrombin generating capacity are not the major causes of ATC. On the other hand, high aPC was associated with increased fibrinolysis and fibrinogenolysis, with high levels of plasmin-antiplasmin (PAP) and D-dimer production. High aPC levels were also strongly associated with mortality, poorer outcome and need for massive transfusion (defined as more than 10 units of RBCs within first 12 hours). Davenport and colleagues concluded that activation of PC is a predominant mechanism of ATC, leading to increased fibrinolytic activity and depletion of fibrinogen. 
Nevertheless, in 2018 Gando and colleagues published a systematic review of the role of aPC in thrombin generation and fibrinolysis in trauma [53], concluding that no studies have shown direct cause and effect relationship between aPC and the suppression of coagulation or increased fibrinolysis.

\section{Hyperfibrinolysis}

Physiological fibrinolysis is a proportionate response to increased fibrin generation in tissue trauma [54]. Following injury, the coagulation and the fibrinolytic systems are both activated, and exist in a dynamic equilibrium [55]. Hyperfibrinolysis is defined as disproportionately increased systemic fibrinolytic activity compared to fibrin formation [56], and is associated with poor clot integrity, excessive bleeding and worse coagulopathy, and increased morbidity and mortality [57].

Hyperfibrinolysis in trauma has not yet been properly defined, and different definitions are used in the literature [55]. However, up to $90 \%$ of severely injured patients have admission biomarker test results indicating some degree of fibrinolysis at some stage after trauma [58]. Plasmin is activated from plasminogen and degrades fibrin. Plasminogen may be activated either by tissue-type or urinary-type plasminogen activators (tPA and uPA, respectively) or by the contact pathway. Both tPA and plasminogen bind to the surface of fibrin which acts as a cofactor for tPA with the net effect being enhanced plasmin generation in the presence of fibrin [59]. Hence, fibrin is in addition to being degraded by plasmin, also an initiator and accelerator of fibrinolysis.

tPA is a serine protease produced and secreted primarily by endothelial cells through endothelial Weidel-Palade bodies in response to vascular activation and shock-induced tissue hypoxia $[30,38,39,60]$. tPA is the primary plasminogen activator [30] activating fibrinolysis. The fibrinolytic activity is tightly controlled by PAI-1 through inhibition of tPA. As aPC 
inhibits PAI-1, activation of PC may therefore induce fibrinolysis through inhibition of PAI-1 [48]. This mechanism is as described above, suggested as an important mechanism of ATC by one of the leading research groups in trauma resuscitation [48].

Platelets constitute the major source of PAI-1. The circulating levels of PAI-1 are low in a normal situation, with large amounts stored within the platelets. Upon platelet activation, PAI-1 is released [60]. However, to what degree platelet transfusion contributes to the inactivation of fibrinolysis remains uncertain [55]. Theoretically, platelet release of PAI-1 may reduce tPA mediated fibrinolysis. A study from Vulliamy and colleagues in 2017 [61] showed reduced fibrinolysis following platelet transfusion with increased levels of PAI-1 and reduced tPA. It remains to be elucidated whether this is related to PAI-1 or other stabilizing components in the platelets [59]. Platelets are in addition to PAI-1, a source of alpha-2antiplasmin ( $\alpha 2 \mathrm{AP})$, thrombin activatable fibrinolysis inhibitor (TAFI) and Factor XIII [60], all promoting clot stabilization [55,59]. $\alpha 2 \mathrm{AP}$ is the principal inhibitor of plasmin, but to what degree $\alpha 2 \mathrm{AP}$ stabilizes the thrombus until lysis is uncertain [60] . It is proposed that $\alpha 2 \mathrm{AP}$ may be more important in downregulating lysis in the initial stages of thrombus formation [60]. Platelets also contain fibrinolytic components like plasminogen and plasminogen activator and the net effect of platelets and platelet transfusion, in different states of platelet activation and how they respond to traumatic injury, continues to be an area of active research $[30,59,60]$.

The aPC theory [48] as the important mechanism of hyperfibrinolysis in ATC has been predominant for the last decade. However, some research networks disagree with the aPC theory, and claim other mechanisms to cause activation of the fibrinolytic system in trauma. Increased tPA release has been suggested as the primary driver of hyperfibrinolysis [62] rather than PAI-1 consumption of aPC. 
Another theory considers ATC to be a fibrinolytic phenotype of disseminated intravascular coagulation (DIC) characterized by activation of the tissue factor-dependent coagulation pathway, insufficient anticoagulant mechanisms, and increased fibrinolysis $[38,55,63,64,65]$. However, the theory of DIC with a fibrinolytic phenotype lacks evidence of intravascular clot formation and fibrin deposition, which is important in DIC [53]. Nevertheless, all the theories agree on a mechanism of shock-induced hyperfibrinolysis by tPA release from activated endothelium $[38,55]$.

\section{Fibrinolytic shutdown}

In 1969 fibrinolytic shutdown was described [66,67] as an acute stress event activating the fibrinolytic system followed by an endogenous inhibition of this system that lasted for days to weeks. Fibrinolytic shutdown measured by Viscoelastic Haemostatic Assays (VHA) in trauma patients was first reported by Moore and colleagues in 2014 [68]. They reported that $65 \%$ of the severely injured patients in their study had low fibrinolytic activity on VHA-tests (defined as Ly30\% below 0,8\%) within 12 hours after injury, and this low fibrinolysis was associated with increased mortality due to organ failure compared to patients with physiological levels of fibrinolytic activity. However, accurate definition of fibrinolytic shutdown in trauma is challenging [67]. Measurement of fibrinolysis degradation products (D-dimer) or complexes (PAP complex) does not necessarily represent the real-time activity as these biomarkers have extended plasma half-lives. Lack of available measurements for real-time reliable fibrinolytic activity has led to a more or less transatlantic on-going debate about the existence of fibrinolytic shutdown as a factor in ATC.

In 2016, the Denver group published results suggesting a fibrinolytic shutdown in up to $46 \%$ of severely injured patients [69]. Moore and colleagues defined 3 different fibrinolytic phenotypes using thresholds for fibrinolysis on VHA: fibrinolytic shutdown (Ly30<0,8\%), 
physiologic fibrinolysis (Ly30 0,81-2,9\%) and hyperfibrinolysis (ly30> 2,9\%). Test results from 2540 severely injured patients were used to group patients into these phenotypes. The most common phenotype was shutdown (46\%), followed by physiologic (36\%) and hyperfibrinolysis (18\%). The hyperfibrinolytic phenotype was more severely injured, required more blood products and accounted for the highest mortality (34\%) followed by shutdown (22\%) and physiologic (14\%). Risk of mortality was significantly increased for hyperfibrinolysis and shutdown compared with physiologic, and there was a survival advantage of physiologic compared to hyperfibrinolysis and shutdown after adjustment for mechanism, systolic blood pressure and severe head injury. However, this survival advantage between physiologic and shutdown disappeared when adjusting for age. The leading cause of death overall was TBI (67\%) followed by haemorrhage (17\%) and MOF (11\%) but cause of death differed significantly between the phenotypes. Death from haemorrhage was $30 \%$ in the hyperfibrinolytic, $14 \%$ in the physiologic and $10 \%$ in the shutdown phenotype, whereas death due to TBI was $71 \%$ in the physiologic, $70 \%$ in the shutdown and $58 \%$ in the hyperfibrinolytic phenotype. There was no survival advantage in the first 24 hours with fibrinolytic shutdown versus physiologic fibrinolysis. Moore and colleagues propose upregulation of PAI-1 as an acute phase reaction, to be important for the transition to fibrinolytic resistance $[67,70]$, but the mechanism has yet to be described.

Gall and colleagues [71] challenged this fibrinolytic shutdown theory in 2018. They looked into the patients who presented low levels of VHA detected fibrinolysis in a study with 914 patients. The survivors in this group were similar to the patients presenting with normal fibrinolysis regarding injury characteristics and outcomes compared to those who died, who were significantly older and more severely injured with a median ISS of 29 and $85 \%$ had TBI. The group with low levels of VHA detectable fibrinolysis who died were nearly 3 times more likely to receive massive transfusion than the survivors, 4 times more likely to be 
coagulopathic with International Normalized Ratio (INR) above 1.2, had extremely high Ddimer levels and their biomarkers were compatible with fibrinolysis. Gall and colleagues found that these patients had high levels of S100A10, especially the group of patients with TBI, and the patients with high levels of S100A10 had much higher mortality compared to those with low S100A10. S100A10 is a membrane-bound receptor which catalyses tPA mediated activation of plasminogen [71]. S100A10 is widely expressed in body tissues and especially in the brain. The S100A10 complex is upregulated as a result of hypoxic stress. Gall and colleagues suggested that S100A10 exposed by tissue injury leads to plasmin generation and hyperfibrinolysis without the need for high levels of circulating tPA. Ex-vivo detection of fibrinolysis depends on the presence of plasmin. Plasmin has a short half-life, and S100A10 is known to increase plasmin autoproteolysis. Gall and colleagues added S100A10 to blood ex-vivo which resulted in reduced VHA detectable lysis in patients with low tPA levels. In patients with high levels of tPA the effect of S100A10 was overcome, and fibrinolysis was detected on VHA. They suggested the S100A10 mechanism to contribute to the serious coagulopathy presented in patients with severe brain injury, without fibrinolysis detected on VHAs.

Moore and colleagues question these findings as the group with low fibrinolysis on VHA (defined as shutdown by Moore) died later than the hyperfibrinolytic patients, they didn't die from bleeding and they had lower rate of massive transfusion [67]. Moore and colleagues suggest that the S100A10 pathway does not necessarily represent active fibrinolysis. They suggest different subphenotypes of the low fibrinolytic activity [67] - the true shutdown or hypofibrinolysis. Trauma patients with true shutdown were at risk of bleeding with deranged coagulation tests, they have had their fibrinolytic system activated after injury, but shut down at the time of admission. The time of transition from fibrinolysis to the proposed shutdown is still unknown, but Moore suggests that the longer the delay from injury to blood sample the 
higher the probability of low fibrinolytic activity. The duration of low fibrinolytic activity after resuscitation is supposed to be a result of the acute phase reaction to prevent excessive bleeding, but at the cost of increased thromboembolic risk, microvascular thrombi and organ failure [67].

The consequence of the controversies regarding hyperfibrinolysis versus fibrinolytic shutdown are not only an academic debate but has caused a transatlantic difference in empirical treatment of bleeding trauma patients with antifibrinolytics. This will be elaborated on further in the Discussion section.

\section{Hypofibrinogenemia}

Fibrinogen is a glycoprotein synthesised in the liver and is the key final component of the clotting cascade, forming fibrin. Low levels of fibrinogen and increased fibrinogen breakdown are key features of TIC $[72,73,74]$, and fibrinogen is the first coagulation factor to become critically low in trauma bleeding [75,76,77]. Fibrinogen levels on admission have been shown to be related to shock, injury severity and mortality [74].

However, the exact mechanism of early fibrinogen depletion is not clear. It is shown that the classical resuscitation components of TIC affect plasma fibrinogen levels. Even though it is not scientifically proven, we believe hypothermia reduces fibrinogen synthesis [78], whereas acidaemia is supposed to lead to increased breakdown of fibrinogen [72]. More recently direct fibrinogenolysis has been postulated as a mechanism [48,59] of hypofibrinogenaemia, with breakdown of fibrinogen driven by the same aPC mechanisms as hyperfibrinolysis [48] . In a study from Davenport from 2017, fibrinogen levels were maintained above critical level when aPC was normal [48]. High levels of aPC were on the other hand, associated with low fibrinogen levels and there was a trend towards fibrinogen reduction to critical levels (below $1,5 \mathrm{~g} / \mathrm{L}$ ) with elevated aPC, suggesting that consumption is not the primary mechanism for 
fibrinogen depletion. Supporting this theory, low fibrinogen levels correlated inversely with plasmin-antiplasmin levels as an indication of fibrinogenolysis, but it was not possible to accurately quantify fibrinogenolysis from fibrinolysis.

\section{Platelet dysfunction}

Platelet dysfunction with reduced aggregation also seems to be a component of the ATC [34]. Impaired platelet aggregation is common after severe trauma and associated with increased mortality [79]. Kutcher and colleagues studied 101 trauma patients with critical injury (mean ISS 23) and demonstrated platelet hypofunction in $45 \%$ of the patients on admission and $91 \%$ had below normal platelet function at some stage during their Intensive Care Unit (ICU) stay, even though platelet counts remained normal. Low Glasgow Coma Score (GCS) and increased base deficit (BD) were significant predictors of platelet hypofunction. Ramsey and colleagues presented in 2016 results from a comparison between 40 patients with blunt injury and 20 healthy volunteers [80]. They found significantly reduced platelets function and activation in trauma patients compared to healthy volunteers, and severity of head injury correlated significantly with platelet dysfunction in a stepwise fashion. This group suggested reduced thrombin-mediated platelet activation as an important component of the platelet dysfunction. However, trauma induced platelet dysfunction is poorly understood and a recent review presents several possible mechanisms of the platelet dysfunction, involving both the coagulation- and the inflammation properties of the platelets [81]. In the future, increased knowledge about platelet dysfunction will hopefully lead to novel therapeutic strategies that may improve outcome. 


\section{Classical trauma triad-hypothermia, haemodilution and acidaemia}

The classical triad of hypothermia, haemodilution and acidaemia is as previously described, associated with TIC [31], but the effect of each of them still involves some uncertainties. Hypothermia at admission is a risk factor for morbidity and mortality in trauma patients $[82,83]$. The effects of hypothermia on coagulation are however not fully understood, but hypothermia has been demonstrated to reduce both platelet function and coagulation enzyme activity [31]. It is also proposed that hypothermia reduces fibrinogen synthesis [78]. Similarly, in a swine model acidaemia following hypoperfusion resulted in increased depletion of fibrinogen and platelets and decreased thrombin generation [31, 84]. A review of the German Trauma Registry database found that pre-admission crystalloid resuscitation was associated with coagulopathy and that the incidence of coagulopathy increased with increasing amounts of crystalloid administered [85]. However, in a study of Davenport and colleagues from 2017, procoagulant factors were minimally affected of a BD above $6 \mathrm{mmol} / \mathrm{L}$ or haemodilution with more than $1000 \mathrm{ml}$ crystalloids [48].

\section{Hypercoagulation}

The body's basis for activation of the endothelium following trauma is to create a prothrombotic environment for wound care. With severe trauma the human organism has to manage the extensive blood loss and massive endothelial trauma simultaneously. With increasing severity of the insult, the progressively more procoagulant endothelium creates a gradient toward anticoagulation to counteract the injury response which ultimately evolves into haemostatic failure [16]. As described above, Johansson and colleagues demonstrated in 2010 how sympathoadrenal activation leads to a dose-dependent surge in circulating catecholamines. Hypercoagulability was demonstrated with moderate catecholamine released followed by hypocoagulation and further to hyperfibrinolysis when catecholamine levels 
increased [42]. The vast majority of trauma patients are mild to moderately injured, and they will be normocoagulable or hypercoagulable on admission to hospital.

For the severely injured patients with initial hypocoagulation, a temporal switch in coagulation status from the initial hypocoagulability of TIC to a hypercoagulable response occurs within hours to days after trauma [86]. In 2018 Sumislawski and colleagues showed $98 \%$ of the patients to be normal or hypercoagulable by 24 hours regardless of primary coagulation status. More than $50 \%$ of the patients were hypercoagulable on day 5 . This study also showed that the occurrence of thromboembolism was associated with failure to initiate thromboprophylaxis within 72 hours.

Hypercoagulability leads to a high risk of thromboembolism after trauma, and the European guidelines on management of major bleeding and coagulopathy following trauma recommend thromboprophylaxis within 24 hours after bleeding control if not contraindicated (most often due to intracranial haemorrhage) [87]. However, studies exploring adequate doses for thromboprophylaxis are still lacking.

\section{Trauma resuscitation in practice}

Management of trauma haemorrhage has undergone a dramatic shift over the past decade. The new understanding of TIC together with new knowledge about resuscitation practices, has radically changed the treatment of severely injured patients, resulting in decreased morbidity and mortality $[8,88]$.

\section{Brief history}

The modern understanding of trauma resuscitation begins in the early 1900s [88]. In the beginning of the World War I, it was thought that blood products caused harm. However, at the end of World War I, whole blood transfusion was becoming safer, due to better tools and 
techniques and increasing experience with casualties receiving transfusions. This increased knowledge about transfusions continued to evolve during World War II and the Korean War. The understanding of the benefits of resuscitation with blood and albumin resulted in increased early survival [89]. However, the mechanisms of shock, the underlying biology and physiologic response to resuscitation were poorly understood, and the surgical care was limited. The increased initial survival was often followed by organ failure, infection and later death [88].

During the Vietnam Conflict field triage, the surgical care as well as the understanding of post injury physiology and biology improved. For the first time pooled banked blood and large volumes of crystalloids were available in a critical trauma setting. Colloids were also developed and introduced in fluid resuscitation. Transfusion of large volumes of blood and crystalloids reduced the mortality and organ failures seen during World War II and the Korean War, but a new entity of a rapid and devastating pulmonary failure - the ARDS, appeared.

Throughout the 1970s and 1980s whole blood was converted to components, and whole blood disappeared from resuscitation. This was caused by concerns about both costs and product waste, and later in the 1980s and 1990s due to the HIV/hepatitis crises [89].

A new understanding of the mechanisms of shock contributed to a paradigm shift in resuscitation in the 1980s/1990s [88]. Trauma resuscitation was pushed towards large volumes of crystalloids and multiple packed $\mathrm{RBC}$ aiming to restore flow and maximize perfusion. The American College of Surgeons, Advanced Trauma Life Support Guidelines (ATLS) [90] suggested all injured patients to have $2 \mathrm{~L}$ of crystalloids before any packed RBC and little attention was given to plasma and platelets. Crystalloids were inexpensive, accessible and easily stored. Among the trauma community the mantra was that plasma and platelets were inherently deleterious, directly causing lung injury and organ failure, and the 
use of crystalloids in trauma resuscitation was rapidly implemented [89]. No evidence supported the opposite and understanding of the trauma biology was lacking. Since plasma and platelets were always administered as part of a large-volume combined resuscitation, it is impossible to discern any direct deleterious effect of any specific component. Another development improving trauma outcome after the 1980s, was the damaged control surgery (DCS), described in 1983 by Stone and colleagues [91], taken further in 1993 by Rotondo and colleagues [92]. The principles of DCS were based upon the recognition that mortality and morbidity in severely injured trauma patients were just as likely to be caused by the physiologic exhaustion from definitive surgery in patients with limited physiologic reserves, as from their actual injuries. The aim of DCS was therefore abbreviated surgery to control lethal bleeding and contamination, while re-establishing peripheral perfusion. This was followed by transfer to an ICU, where the physiology was restored, and the patient was rewarmed. When the physiology was deemed normal, the patient underwent definitive surgery $[93,94]$. Soon DCS became the standard of care in massively bleeding trauma patients [94]. This new surgical principle together with large volume resuscitation led to increased survival, but with a significant increase in MOF.

In the early 2000s management shifted radically towards the current understanding of trauma resuscitation $[95,96]$. The two main reasons were the understanding and characterisation of TIC [21,22] together with the first descriptions of haemostatic resuscitation [89,97]. It was becoming clear that large volumes of crystalloids were worsening the coagulopathy through dilution, contributed to metabolic acidosis and exacerbated hypothermia if infused cold [89]. This radical shift emphasized balanced transfusion with blood product, using ratios that approximated whole blood, limiting use of crystalloids, using permissive hypotension and DCS. The concept was named damage control resuscitation (DCR) [98]. 


\section{Current practice}

\section{Immediate Management}

All patients need to be resuscitated from shock to restore normal organ perfusion. However, patients who are shocked, may not have an ongoing bleeding, and patients who are actively bleeding may not yet have developed shock. Early identification of ongoing bleeding is a key step in trauma management [34].

Peripheral vasoconstriction preserve blood when circulating volume is lost, but can potentially lead to inadequate organ perfusion even though blood pressure remains normal. In trauma, inadequate cardiac output and organ perfusion are not necessarily associated with hypotension, only when blood loss is critical or rapid is there a relationship between the two [34].

However, due to the lack of objective parameters, The ATLS classification by the American College of Surgeons still suggests the use of clinical measures to identify bleeding patients and to estimate blood loss [90].

Another suggested approach to identify bleeding trauma patients, has been to give the patient a volume bolus and assess the response. If the patient has signs of bleeding and is a nonresponder or transient responder to the volume bolus, the patient should be expected to be actively bleeding and treated according to DCR protocols [99]. However, this strategy is controversial [34]. A bolus of crystalloids will dilute and potentially worsen coagulopathy, while using blood products from the beginning may cause unnecessary transfusions in some patients.

Various scoring systems have been developed to try to identify bleeding patients $[34,99,100]$, but still none of them have been reported as sufficiently robust to reliably predict massive transfusion [101]. 
A practical approach to identify trauma patients with ongoing bleeding is therefore a combination of physiology, severity of injury and response to initial resuscitation, requiring multidisciplinary teamwork and communication [34].

\section{Damage Control Resuscitation (DCR)}

DCR directly addresses the lethal triad [97] and mainly consist of DCS, haemostatic resuscitation and deliberate hypotension $[34,89,99,102]$. DCS mandates controlling the bleeding and contamination [96]. Haemostatic resuscitation includes blood products in a balanced ratio approximating whole blood and avoiding crystalloids. Hypotensive resuscitation implies accepting an adequate, lower than normal, blood pressure while the patient is actively bleeding, aggressively restoring perfusion as soon as haemostasis has been achieved.

\section{Permissive hypotension}

The concept was introduced as early as 1994, when Bickell and colleagues reported improved survival and better outcomes for penetrating torso injuries who received no fluid until arrival in the operating room [103]. Several animal studies have also shown benefits from permissive hypotension [104]. Dutton and colleagues demonstrated in 2002 no mortality differences nor harm between a group of haemorrhaging trauma patients with a systolic blood pressure of 70mmHg versus $100 \mathrm{mmHg}$ [105]. Consequently, the concept of permissive hypotensive resuscitation has been widely accepted and implemented, even though strong medical evidence is lacking. Both the European guidelines on management of major bleeding [87] and the National Institute of Health and Care excellence (NICE) guidelines [101] support hypotensive resuscitation targeting a systolic blood pressure of $80-90 \mathrm{mmHg}$ for actively bleeding trauma patients without TBI. Recently, a systematic review and meta-analysis of 
hypotensive versus conventional resuscitation of adult trauma patients with haemorrhagic shock, concluded that permissive hypotension may offer a survival benefit and reduce blood loss and blood product requirement, but most studies were underpowered, and there is need for stronger evidence [106]. Permissive hypotension is however contraindicated in patients with TBI and coronary artery disease [31].

The ideal blood pressure remains unclear. Higher blood pressure than what is required for adequate oxygen requirement and organ perfusion, might be harmful and is not desirable during ongoing bleeding [34]. Increased blood pressure might increase bleeding and the products given to increase the blood pressure have adverse effects and aggravate existing coagulopathy. Even blood products in a balanced ratio will result in some degree of dilution [34].

In practice it might be difficult to target a specific blood pressure during resuscitation and ongoing bleeding. Measurement of systemic parameters of organ perfusion, like lactate and base excess/deficit, gives an estimation of the severity of shock. In trauma patients without pre-existing comorbidity, there is a strong correlation between the standard parameters of lactic acidosis and prediction of outcome [34]. Correction of lactate and BD is therefore being used as a measure of response to resuscitation. A drawback is that arterial blood gas tests are not continuous and dependent on blood draws. Other techniques for continuous measuring of the severity of shock and organ perfusion have been investigated, but currently the most available continuous measure is end-tidal $\mathrm{CO}_{2}$ in ventilated patients, where decreased perfusion is reflected in decreased end-tidal $\mathrm{CO}_{2}[34]$.

Vasopressors have been hypothesized to counteract vasoplegia following cytokine release, nitric oxide products, cellular, mitochondrial and tissue damage and reperfusion injuries related to the shock. However, vasopressors have a wide range of potential adverse effects, 
and the conclusion of published studies suggests increased mortality associated with vasopressors [34]. Currently, there is no evidence to include vasopressors in the initial resuscitation of trauma bleeding, and adequate blood pressure to maintain organ perfusion should be managed with blood products and surgical intervention.

\section{Massive Transfusion Protocol (MTP) and haemostatic resuscitation}

A MTP is an integral part of DCR and provides guidelines to clinicians on how to best deliver transfusion therapy to bleeding trauma patients. Since the development of MTP, several studies have been evaluating outcomes from different transfusion ratios. Malone and colleagues suggested in 2006 a 1:1:1 ratio of RBC: plasma: platelets to be the optimal treatment to best prevent coagulopathy and seemed to be associated with improved outcomes [107]. In 2008 Holcomb and colleagues published results from an observational multicentre study showing that plasma/RBC and platelet/RBC ratios greater than $1: 2$ improved survival [108]. The same research group published in 2013 results from a Prospective, Observational, Multicentre, Major Trauma Transfusion (PROMMTT) study of near 1000 trauma patients, showing that higher plasma and platelet ratios early in trauma resuscitation decrease mortality in patients who received more than 3 units of RBC [109]. These and several other observational studies $[110,111,112,113,114,115]$ focusing on transfusion ratios formed the basis for the need of a randomised controlled trial (RCT) on transfusion ratios. The Pragmatic, Randomized Optimal Platelet and Plasma Ratios trial (PROPPR) randomised patients with trauma predicted to receive massive transfusion (defined as more than 10 units of RBC first 24 hours) to a ratio of plasma:platelets;RBC of $1: 1: 1$ versus $1: 1: 2$. No significant differences between the two groups were found for 24-hour or 30-day mortality, but the 1:1:1 group had fewer early deaths caused by exsanguination, they had improved rates of achieving haemostasis and there were no differences in adverse events [116]. These findings led to the 
1:1:1 ratio of blood products becoming a widely accepted strategy in trauma resuscitation $[34,89,99,102]$. This is implemented into both the European Guidelines on management of major bleeding and coagulopathy following trauma [87] and the latest NICE guidelines [101].

High ratios of plasma seem to benefit trauma patients. Plasma has been shown to reduce dilutional coagulopathy of trauma [117] and is rich in clotting factors necessary for thrombin generation. Experimental rodent studies have also shown that plasma can repair and normalize the vascular endothelium by restoring tight junctions, restoring endothelial glycocalyx and inhibiting shedding of glycocalyx [40]. The effects of plasma in decreasing inflammation and oedema, and hence, reduce organ failure, have been demonstrated in rats [118]. The Targeted Action for Curing Trauma Induced Coagulopathy (TACTIC) research group published in 2017 results showing that high ratios of plasma to RBCs were associated with lower transfusion requirement and mortality [119]. Recently, mortality improvement has also been shown comparing prehospital plasma versus crystalloids in an RCT including 500 patients [120]. Another recently published study showed improved outcomes in patients with TBI receiving plasma prehospital compared to those receiving only RBCs [121]. However, simultaneously, a similar smaller RCT with 114 patients concluded that use of prehospital plasma was not associated with survival benefit, but in this study transport time to a trauma centre was less than 30 minutes.

At present, plasma is increasingly used in trauma resuscitation, during and after haemostasis has been achieved, but still evidence of the benefit is sparse.

Plasma is available as fresh frozen plasma (FFP), produced in local blood banks, or as Octaplasma ${ }^{\circledR}$ (Octapharma AS). FFP is prepared from single donor whole blood and stored frozen, while Octaplasma ${ }^{\circledR}$ is pooled plasma, from approximately $600-1500$ donors, treated 
with solvent-detergents to potentially eliminate virus, bacteria and parasites. Pooling plasma increases safety by diluting antibodies to human neutrophil antigens and human leukocyte antigens to non-clinically significant amounts, and thereby preventing immune responses [122]. Octaplasma ${ }^{\circledR}$ has also shown other beneficial effects above FFP. VIPER-OCTA, a randomized, clinical pilot trial from Denmark on patients undergoing emergency surgery for thoracic aorta dissection, found reduced glycocalyx and endothelial injury, reduced bleeding, transfusions and use of haemostatic therapy, and time on ventilator for patients receiving Octaplasma ${ }^{\circledR}$ compared to FFP [123]. In Norway, we have been using pooled plasma as our plasma-product since 1992, except for some prehospital services using freeze-dried plasma for logistical reasons over the latest years.

Platelet transfusion is recommended as part of the MTP. Intuitively, since platelet function is known to be compromised after injury, platelet transfusion should improve the haemostatic ability of bleeding patients. Holcomb and colleagues presented in 2011 a retrospective study showing survival benefits with high ratios of platelets: RBC (defined as 1:1). The TACTICgroup published in 2017 results showing a strong relationship between high ratios of platelets to RBCs and reduced requirement of transfusions and mortality [119]. Furthermore, in a substudy of the PROPPR trial, they found that patients who received platelets early, which in PROPPR was before plasma and RBCs, had lower 24 hours - and 30-days mortality, even when controlling for plasma volume transfused [124]. In addition, more patients in the platelets group achieved haemostasis and fewer died from exsanguination.

However, the evidence for a beneficial effect of platelets transfusion is limited [99]. Platelet counts are often normal during trauma resuscitation, and the understanding of the effects of platelet transfusion is limited [81]. 
A study from Vulliamy and colleagues in 2017 didn't show any improvement in platelet aggregation following platelet transfusion, but they discovered reduction of hyperfibrinolysis [61], and the effect of platelets on fibrinolysis is a field of growing interest [60].

Platelet transfusion is not without risks, and account for a large proportion of transfusion reactions [81]. In addition, platelets are difficult to store and have a short lifetime compared to other blood products, which makes them a vulnerable resource.

Nevertheless, based on today's knowledge, platelets are an important part of the MTP, both as part of a balanced ratio of blood products, and added when required as a result of coagulation tests.

\section{$\underline{\text { Tranexamic Acid (TXA) }}$}

TXA is a lysine analogue and inhibits the enzymatic breakdown of fibrin blood clots. The fibrin breakdown begins when plasminogen, a glycoprotein pro-enzyme produced by the liver, binds to strands of fibrin via lysine binding sites. tPA released by the vascular endothelium binds to fibrin and converts plasminogen to active plasmin. Once formed, plasmin cleaves fibrin into small protein fragments (fibrin degradation products) such as Ddimers. This cleavage exposes more lysine residues binding more plasminogen thus initiating positive feedback that accelerates fibrinolysis. If the lysine residues on fibrin are enzymatically removed, plasminogen binding is inhibited. TXA has a molecular structure like lysine inhibiting fibrinolysis by preventing the binding of plasminogen to fibrin. TXA has also been shown to inhibit fibrinogenolysis [125], probably due to the same mechanism.

Despite some disagreement concerning hyperfibrinolysis versus fibrinolytic shutdown, hyperfibrinolysis is considered relatively common after severe injury, and correction of this is part of the treatment of coagulopathic patients [58]. TXA as an antifibrinolytic to trauma 
patients was studied in CRASH-2 [126,127,128]. This large, multicentre RCT with more than 20.000 patients presented significantly reduced mortality for patients receiving TXA less than 3 hours after injury, without increase in adverse events. The greatest effect on the mortality was when TXA was given within 1 hour after injury [129], and with potentially deleterious effects when given later than 3 hours after injury [129]. This survival benefit has been reproduced in the military studies MATTERS and MATTERS-2 [130,131], with an augmented additional beneficial effect of cryoprecipitate in MATTERS-2. In contrast to CRASH-2 the MATTERs study reported an associated risk of thromboembolism in the TXA subjects but concluded that this greater risk should be attributed to the higher injury severity in the TXA subjects. When evaluated by multivariate analysis, adjusting for severity of injury, no independent association between TXA use and thromboembolic events was found. More recent studies also support the effect of TXA. In 2015, Cole and colleagues showed reduced rates of organ failure and outcome benefits of TXA given empirically as part of MTP when expecting bleeding [132]. The TACTIC group showed in 2017 a strong association of reduced transfusion requirement and mortality when TXA was given empirically as part of MTP [119].

In a Cochrane review from 2015 on antifibrinolytic drugs to trauma patients, the authors concluded that "TXA safely reduces mortality in trauma patients with bleeding without increasing the risk of adverse events. TXA should be given as early as possible and within three hours of injury, as further analysis of the CRASH-2 trial showed that treatment later than this is unlikely to be effective and may be harmful" [133].

Following this, TXA has pragmatically been provided to trauma patients in the prehospital environment. However, the effectiveness and safety of TXA prehospital has been poorly studied, and the first multicentre placebo-controlled RCT studying TXA given prehospitally was published in 2020 [134]. A total of 900 patients were included, no difference was found 
in mortality, transfusion requirement, MOF or adverse events comparing TXA prehospital with placebo. Similar to CRASH 2 this study found a reduced mortality in the TXA subgroup when TXA was given within 1 hour after injury, and in a small subgroup of patients with severe shock.

Today, both the European Guidelines on management of major bleeding and coagulopathy following trauma [87] and the NICE guidelines [101] recommend empiric administration of $1 \mathrm{~g}$ of TXA as a bolus followed by a continuous infusion of $1 \mathrm{~g}$ TXA over the next 8 hours to all patients with high risk of bleeding if started within 3 hours after injury, and is widely incorporated into local MTPs, especially in Europe [55].

However, empiric TXA remains controversial following the ongoing disagreement of hyperfibrinolysis versus fibrinolytic shutdown. The dosing regimen also needs re-evaluation.

\section{Individualized goal-directed therapy}

Following better knowledge of the pathophysiology of the coagulopathy of trauma, a modern understanding of the haemostatic resuscitation has been developed, often referred to as the "Copenhagen concept". The concept was introduced by Per I. Johansson from Rigshospitalet in Copenhagen in 2005 [135], and has been widely implemented in trauma resuscitation protocols during the last decade. The aim of this transfusion regimen is to maintain normal coagulation during the resuscitation phase. The concept suggests that when a patient is bleeding uncontrollably, massive transfusion starts with a balanced ratio of blood products, while collecting blood samples for coagulation monitoring. Johansson and colleagues used VHA to monitor coagulation, but the strategy may also include use of conventional coagulation tests (CCTs), depending on which modality is available. When test results are available, adjustment of the haemostatic resuscitation with additional haemostatic therapy is 
added. This strategy allows an early shift toward individualized haemostatic goal-directed therapy and is today the gold standard of modern trauma resuscitation [99].

Therapy administered according to test results in trauma consists of fibrinogen concentrate and/or cryoprecipitate and additional platelets, plasma or TXA on top of the balanced transfusion ratio dictated by the local MTP [102].

\section{$\underline{\text { Fibrinogen }}$}

Fibrinogen is a fundamental substrate for haemostasis. TIC is associated with low fibrinogen levels on admission [73], which in turn has been associated with increased mortality and transfusion requirements [74,136,137,138]. Hagemo and colleagues showed in 2014 dramatically increased mortality with fibrinogen below $2.29 \mathrm{~g} / \mathrm{L}$ on admission [72]. Augmentation of blood fibrinogen levels necessitate use of a concentrated form of fibrinogen supplementation, as plasma substitution will not normalize a low fibrinogen level [73,117,139]. Theoretically, fibrinogen substitution seems beneficial in trauma resuscitation, and early fibrinogen replacement is safe $[75,99]$. However, clinical benefit of empirical early fibrinogen replacement has not been proven yet [99]. CRYOSTAT-2 [140] is an ongoing, pragmatic, multicentre trial, randomising bleeding trauma patients to either early cryoprecipitate in addition to a standard MTP versus standard MTP. The aim of CRYOSTAT2 is to identify the effect of early fibrinogen substitute.

Based on existing knowledge, the European guidelines on management of major bleeding and coagulopathy following trauma recommend fibrinogen levels above 1.5-2.0g/L [87].

Current concentrated fibrinogen sources are cryoprecipitate and fibrinogen concentrate. Both cryoprecipitate and fibrinogen concentrate have advantages and disadvantages [141]. Fibrinogen concentrate is heat-treated lyophilized fibrinogen powder made from pooled 
human plasma, while cryoprecipitate is made from controlled thawing of frozen plasma, and in addition to be a concentrated source of fibrinogen, also contains other coagulation factors like Factor VIII, von Willebrand factor and Factor XIII [142].

As cryoprecipitate is a blood product, it carries the risk of transfusion reactions and transmission of infectious diseases, while fibrinogen concentrate undergoes viral inactivation. Cryoprecipitate has a short shelf life after thawing that might lead to wastage, while fibrinogen concentrate is reconstituted for immediate use, and the wastage is negligible $[141,142]$. Currently, there are no clear clinical data showing that fibrinogen concentrate is superior to cryoprecipitate in any clinical setting [142]. However, fibrinogen concentrate is more expensive than cryoprecipitate [141].

The optimal dosing of fibrinogen supplementation remains unclear, and the recommendations vary [143]. A dose of $1 \mathrm{~g}$ fibrinogen has been suggested to increase fibrinogen concentration with approximately $0.25 \mathrm{~g} / \mathrm{L}$ and increase FIBTEM (ROTEM assay) Maximum Amplitude (MA) with 1.5 to $2 \mathrm{~mm}$ [143]. Accurate dosing is more feasible with fibrinogen concentrate than cryoprecipitate, due to variability of fibrinogen concentration in cryoprecipitate. To maintain a plasma fibrinogen above $2 \mathrm{~g} / \mathrm{L}$, trauma patients may occasionally require relatively high doses of fibrinogen [119], and there have been concerns about thromboembolic complications associated with fibrinogen concentrates. Currently there is no evidence to support increase in thromboembolic events when treated with fibrinogen concentrate [143,144] or cryoprecipitate. In 2017 Schlimp and colleagues demonstrated that patients treated with fibrinogen concentrate did not have higher plasma fibrinogen levels than a control group in the post trauma phase up to day 7 [145]. They demonstrated a rise in plasma fibrinogen levels post trauma in all trauma patients and proposed the mechanism to be increased hepatic synthesis as part of the acute phase response to severe trauma. This is 
supported by the CRYOSTAT-1 study, the E-FIT-1 and the FiiRST trial, which all presented rise in post trauma plasma fibrinogen levels, but no increased risk of thromboembolism following cryoprecipitate therapy [146] or fibrinogen concentrate [147,148].

\section{Detection and monitoring TIC}

Since ATC was described in 2003 , efforts have been made to identify a clinically relevant test and algorithm to guide haemostatic therapy. The perfect coagulation test should have high sensitivity and specificity. It should target all aspects of the haemostasis, including both the procoagulant and the anticoagulant arms. It should help individualizing therapy for a given patient and monitor the response to treatment. It should also be simple, rapid, broadly interpretable, and inexpensive. That perfect laboratory test does not exist and there are no broadly accepted standard laboratory method to identify and prognosticate TIC [35,39].

In the first years after 2003, the identification of the coagulopathic patients was based on traditional transfusion triggers recommended by massive transfusion guidelines [74]. In 2010 Frith and colleagues showed, in a multicentre study of more than 3600 trauma patients, that an admission PTr above 1.2 was associated with higher mortality and transfusion requirements [74]. They recommended PTr to be adopted as a clinically relevant definition of ATC. In 2015 Peltan and colleagues showed in more than 1300 trauma patients, a significantly increased risk of transfusion requirements and mortality with an INR above 1.5 compared to 1.2. They suggested INR above 1.5 to be adapted as an identifier of ATC [149]. Currently, a patient is defined as hypocoagulopathic if the INR is determined to be above a specific value; typically, 1.2 to 1.5 (for patients not on prescribed anticoagulants), based on the evidence showing that with increasing values, patients require more transfusion and suffer higher mortality and morbidity [33,39]. 
Laboratory coagulation tests like INR/ PTr and aPTT, are available worldwide as low-cost standardised tests. However, they have disadvantages in acute trauma care. These CCTs are plasma tests reflecting only some of the coagulation factors and provide no information on whole blood clot kinetics or the platelet contribution [17]. In addition, most CCTs are time consuming [150]. More rapidly available Point-of-care (POC) INR-tests have been proposed for identifying coagulopathic patients and have shown good correlation with rapid thromboelastography values (r-TEG) and transfusion requirement [151]. However, other studies have shown only modest agreement and correlation with laboratory INR [152].

The lack of a precise laboratory tests identifying TIC, has led to renewed interest for VHA. Over the last decade, numerous studies have suggested different threshold values, algorithms and definitions of TIC and ATC for VHA in the trauma setting. Although it takes as long time as the CCTs to have complete VHA traces, clinically relevant parameters can be obtained in about 10 minutes, to guide early needed haemostatic therapy.

\section{Viscoelastic Haemostatic Assays (VHA)}

VHA has been used in cardiac surgery and liver transplants for decades [153], but formal use in trauma dates to a case series published in 1997 [154]. Kaufmann and colleagues evaluated 69 trauma patients with TEG, and classified patients as hypo-, normal- or hyper-coagulable. They concluded that TEG was useful in determining coagulation abnormalities and was an early predictor of transfusion requirements after blunt trauma.

Since then, the enthusiasm and use of these devices in trauma has increased worldwide, to levels beyond the relatively sparse evidence. 


\section{$\underline{\text { Brief description of VHA }}$}

The most common VHA devices today are the TEG® (Thromboelastography) and ROTEM® (Rotational Thromboelastometry). The best studied TEG device is the TEG5000® Thrombelastograph Haemostasis Analyzer (Haemonetics Corp., Braintree, MA, US), but recently, a modern system device has been introduced (TEG 6S). For ROTEM the best studied device is the ROTEM Delta ${ }^{\circledR}$ (formerly TEM international GmbH, Munich, Germany, now Instrumentation Laboratory, Bedford, MA, USA), but a modern version has been introduced (ROTEM Sigma).

Comparing the thromboelastography device to the thromboelastometry device, no evidence supports the superiority of either device in trauma per today [35].

The methodology of TEG and ROTEM is complex. Briefly described, the principle of the devices is based on a method with a cup and a pin to measure the mechanical properties of the clot formation in whole blood. The cup or the pin moves relative to the other part, and as the clot form, the resistance to movement built up by fibrin strands, is translated into a graphical presentation $[35,155,156,157,158]$. Different activators are added to examine different aspect of the clot formation. The traces presented can be broadly divided into 4 different stages [156];

- time to first fibrin formation

- Reaction time (R) or activated clotting time (ACT - calculated value, not shown in figure) in TEG

- Clotting time (CT) in ROTEM

- kinetics of the fibrin polymerisation

○ $\alpha$-angle in both TEG and ROTEM

o time from first fibrin formation to the clot is $20 \mathrm{~mm}$ wide: 
- kinetics time $(\mathrm{K})$ in TEG

- clot formation time (CFT) in ROTEM

- clot strength at different time points

○ amplitude (A) in TEG

- clot amplitude (CA) in ROTEM

○ with maximum clot strength at the peak amplitude

- maximum amplitude (MA) in TEG

- maximum clot firmness (MCF) in ROTEM

- clot breakdown/lysis

$\circ$ presented as a percentage of reduction compared to the maximum amplitude at different timepoints for TEG (Lysis (Ly))

- presented as percentage clot remaining of MCF in ROTEM (clot lysis/ lysis index/Maximum lysis (CL/Li/ML))

TEG

ROTEM

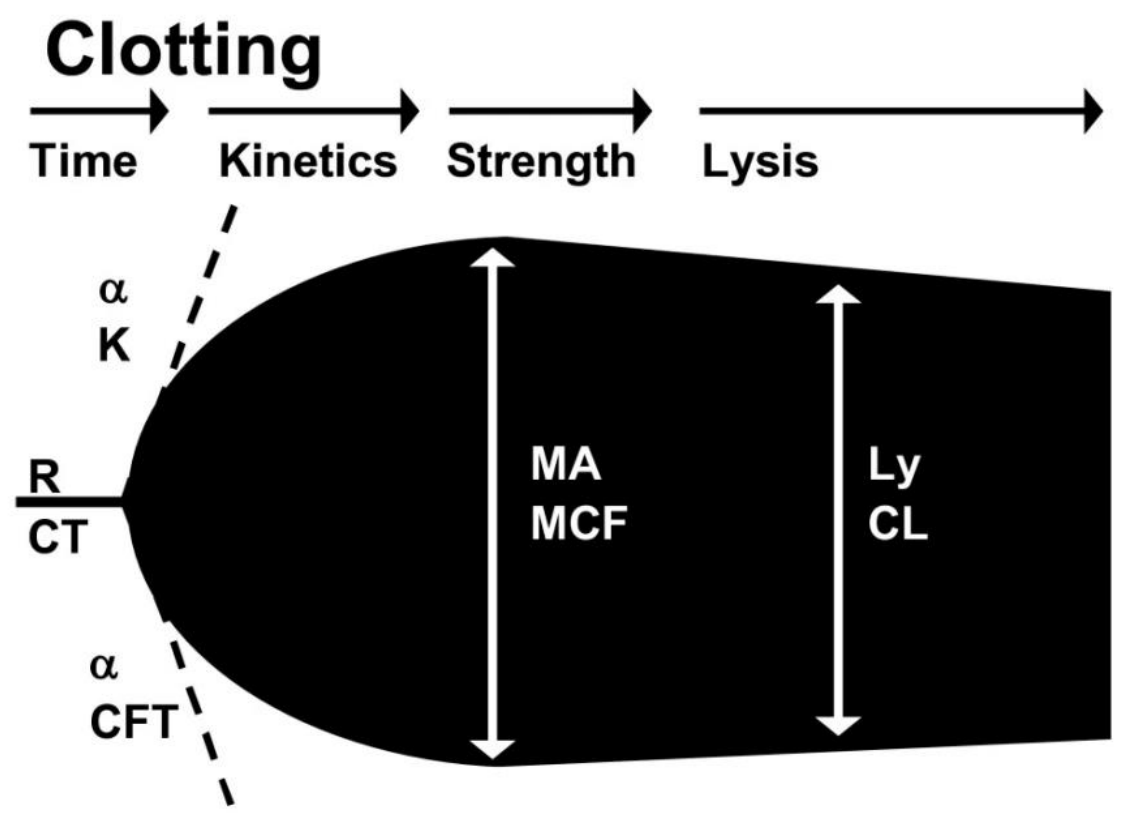

Schematic TEG (upper part)/ROTEM (lower part) trace indicating the commonly reported variables reaction time (R)/clotting time (CT), clot formation time (K, CFT), alpha angle ( $\alpha$ ), maximum amplitude (MA)/maximum clot firmness (MCF) and lysis (Ly)/clot lysis (CL).

Copy from Johansson [156], re-printed with permission from Springer Nature. 
The most common activators added for use in trauma is the extrinsic pathway activation with TF, named Rapid TEG (rTEG) or EXTEM, the intrinsic contact activation pathway, named Kaolin TEG or INTEM, and the fibrinogen assays, named Functional Fibrinogen (FF) TEG or FIBTEM. FF TEG is activated by lyophilized tissue factor and a potent platelet inhibitor Abciximab (a glycoprotein IIb/IIIa- inhibitor) is added, whilst FIBTEM is activated with TF derived from rabbit brain with cytochalasin $\mathrm{D}$ added as the platelet inhibitor to isolate the fibrin component of the clot.

The first step in the VHA trace, the time to first fibrin formation, reflects the clotting factors. A prolonged R-time or CT may be due to deficiencies in the coagulation factors. Kaolin TEG and INTEM seems to be superior to the tissue factor activation in examining the function of the clotting factors [35].

Clot formation occurs from the start of fibrin polymerization until the clot reaches the maximum strength. This is largely dependent on fibrinogen, but $\alpha$-angle, K-time and CFT are confounded in settings with low platelet and low thrombin generation. Fibrin-specific assays (FF TEG and FIBTEM) were developed to improve the accuracy of VHA in detecting fibrinogen deficiencies. Currently, laboratory fibrinogen is still the gold standard for measure blood fibrinogen [35]. However, laboratory-based fibrinogen measurements take up to an hour, far too long in critically bleeding patients [75]. Therefore, VHAs, and especially the fibrinogen assays, are increasingly used to measure fibrinogen activity and need for fibrinogen substitution in trauma [35].

Reduced MA or MCF is often treated with platelets. However, the platelet function is poorly reflected in standard VHA test, although up to $80 \%$ of the MA or MCF is due to platelets. TEG provides special reagents to examine platelet function - platelet mapping. Currently, there is no evidence for use of platelet mapping in trauma. ROTEM has no VHA reagents to 
examine platelet function, but the manufacturer of ROTEM provides a special device to measure platelet aggregation using impedance aggregometry. At present, no evidence supports its use in trauma.

Up to date, VHAs are the only tests available to diagnose hyperfibrinolysis in a clinically useful timeframe for trauma resuscitation [59]. D-dimer is available as a CCT, but D-dimer levels alone are incapable of predicting VHA-detectable fibrinolysis [59]. Various definitions of hyperfibrinolysis for VHA have been described [55]. A ML of 15\% for ROTEM [159] and a Ly30 of 3\% for TEG [160] have been suggested and are commonly used thresholds. However, in a study from Raza and colleagues in 2013 , up to $90 \%$ of severely injured patients had biomarker test results at admission suggesting some degree of fibrinolysis and $57 \%$ had evidence of "moderate" fibrinolysis, despite only $5 \%$ had a ML above $15 \%$ on ROTEM [58].

\section{VHA in trauma}

Following the increasing enthusiasm for VHA, numerous papers have been published during the last decade to support VHA in trauma and try to describe their superiority over CCTs in guiding trauma resuscitation. This growing number of papers has been followed by several recent comprehensive systematic reviews $[35,153,157,161,162,163,164,165]$, concluding that there is limited support for the use of VHA in trauma. A Canadian review from 2014 [162] concluded that limited evidence from observational data suggested that TEG/ ROTEM tests could diagnose TIC and might predict transfusions and mortality in trauma. However, the effects on blood product transfusions, mortality and other outcomes remains unproven in RCTs. The same group investigated in a systematic review in 2016 [165], thresholds for ROTEM parameters in diagnosing coagulopathy, predicting and guiding transfusions and predicting mortality. They found indications that abnormal EXTEM and FIBTEM values could diagnose coagulopathy and predict blood transfusions and mortality. However, due to 
arbitrary cut-off values, lack of RCTs, cohort studies with small sample sizes without comparable controls and heterogeneous patient populations, no further conclusions could be drawn from the literature to date. In 2015, a NICE-report from Whiting and colleagues [161] concluded that VHA is cost-saving and more effective than standard laboratory tests in cardiac surgery patients, but there was no data on clinical effectiveness in trauma, even though the VHAs were suggested to be cost saving. In a Cochrane review from the same year [163] the conclusion strongly suggested that VHA should be used for research only. Wikkels $\varnothing$ and colleagues concluded in another Cochrane review from 2016 that "there is growing evidence that application of TEG- or ROTEM-guided transfusion strategies may reduce the need for blood products and improve morbidity in patients when bleeding. However, these results are primarily based on trials of elective cardiac surgery involving cardiopulmonary bypass, and the level of evidence remains low. Further evaluation of TEG-or ROTEM-guided transfusion in acute settings and other patient categories in low risk of bias studies is needed" [153]. A meta-analysis of fifteen RCTs (nine trials referred to cardiothoracic patients, one to liver transplantation, one to surgical excision of burn wounds and one to trauma) conducted by Fahrendorff and colleagues in 2017 [164] showed that the amount of transfused RBCs, FFPs and bleeding volume was significantly reduced in the VHA-guided groups, whereas no significant difference was found for platelet transfusion requirements or mortality. They concluded that the VHA algorithms showed a trend towards superiority, but larger RCTs were needed. In 2018, new guidelines for the British Society for Haematology, conducted by Curry and colleagues, concluded that "Viscoelastic haemostatic assays devices have practical advantages as point-of-care devices for monitoring major haemorrhage including speed of results and a set of parameters that assesses a global coagulation profile. However, the lack of a systematic approach to their use, with low quality published data that has not been clearly linked to important clinical outcomes means that, at present, the evidence base to 
guide practice is limited" [157]. Blaine and colleagues, published in a review in 2019 that TEG and ROTEM are imperfect, but not useless in trauma resuscitation and that VHAs can provide additional diagnostic information in a confusing and chaotic environment [35]. The clinicians should focus on those parameters that provide the greatest diagnostic discrimination, and VHA can be preferable to INR and aPTT. Nevertheless, clinicians should look to fibrinogen and platelet counts when available. The authors questioned the possibility to ever design and execute a meaningful prospective trial for interventions in the heterogenous trauma population. Most recently, in 2020, the Eastern Association for the Surgery of Trauma (EAST) published guidelines for practical use for TEG and ROTEM in bleeding patients with coagulopathy [166]. They conditionally recommend TEG/ ROTEMguided strategy versus non-TEG/ ROTEM strategy to adult trauma patients with ongoing haemorrhage and concern for coagulopathy. As stated by the authors, the recommendation is based on very low level of evidence and justified by potential benefit of fewer patients being exposed to blood products and reduced mortality combined with lack of harm to the patients from using TEG/ ROTEM.

Despite limited evidence, the enthusiasm for VHA in trauma is maintained. The growing understanding of TIC and personalization of trauma resuscitation demand coagulation tests customized for trauma, and targeted treatment in the chaotic trauma resuscitation setting require meaningful algorithms that are easy to comprehend. VHAs are dynamic tests, performed bedside with first test results available within minutes, and thus seems to satisfy the needs of the coagulation monitoring in trauma compared to the time consuming CCTs. Over the last decade, trauma centres worldwide have therefore developed resuscitation algorithms for trauma, including VHA guiding of haemostatic therapy $[135,167,168,169,170]$. 
Most of these algorithms are based upon reference values from the manufactures or expert or personal opinions, and none of them have been validated for trauma. 


\section{AIM AND RESEARCH QUESTIONS}

As thoroughly discussed in the Introduction, trauma resuscitation has evolved dramatically over the last two decades with the introduction of DCR with MTPs and haemostatic therapy. Due to increased understanding of TIC and its treatment, transfusion strategies including individualized goal-directed therapy have over the last years, been widely adopted as state-ofart in trauma resuscitation. However, evidence-based resuscitation algorithms are lacking, and little evidence exists on how to best monitor coagulation status during trauma haemorrhage. This knowledge gap initiated the research presented in this thesis.

\section{Paper 1}

The aim of this study was to use a large cohort from an international observational multicentre study of trauma patients to define clinically useful VHA and CCT parameters and threshold values to identify coagulation deficiencies in haemorrhaging trauma patients and to guide haemostatic therapy.

\section{Papers 2 and 3}

The primary aim of this study was to address the lack of evidence-based knowledge and RTCs, on the effects of VHAs versus CCTs in guiding goal-directed individualized resuscitation in trauma haemorrhage. Bleeding trauma patients were randomized to an empiric, evidence based, best practice MTP including TXA, followed by individualized haemostatic therapy guided by algorithms using either VHAs or CCTs to monitor coagulation during bleeding. 


\section{MATERIALS AND METHODS}

\section{Design}

The included studies were part of the "Targeted Action for Curing Trauma Induced Coagulopathy" (TACTIC) program, funded by the European Commission under the FP-7 HEALTH-Contract No. F3-2013-602771. The TACTIC Program was conducted by International Trauma Research Network (INTRN) and involved 6 major trauma centres in Europe during the period November 2014 to May 2019. Both TEM® International GmbH and Haemonetics ${ }^{\circledR}$ were equal partners in the TACTIC program and provided VHA devices and reagents for all participating institutions. The funders had no role in the study design, data collection and analyses, decision to publish, or preparation of manuscripts presenting results from the program.

\section{Paper 1}

Data collected in the prospective international, observational multicentre study ACIT (Activation of Coagulation and Inflammation in Trauma) was the research platform for this study. Patients were recruited at 6 major trauma centres in 5 different countries in Europe The Royal London Hospital (London, United Kingdom (UK)), John Radcliffe Hospital (Oxford, UK), Academic Medical Centre (Amsterdam, Netherlands), Rigshospitalet (Copenhagen, Denmark), Kliniken der Stadt Köln gGmbH (Cologne, Germany) and Oslo University Hospital (Oslo, Norway).

\section{Paper 2 and 3}

The ITACTIC (Implementing Treatment Algorithms for the Correction of Trauma-Induced Coagulopathy) study was an investigator-initiated, pragmatic, multi-centred, superiority, parallel-group, RCT performed at 7 major trauma centres in Europe. The trial sites included 
Rigshospitalet (Copenhagen, Denmark), Academic Medical Centre (Amsterdam, The Netherlands), Oslo University Hospital (Oslo, Norway), Kliniken der Stadt Köln gGmbH (Cologne, Germany), The Royal London Hospital (London, UK), John Radcliffe Hospital (Oxford, UK) and Nottingham University Hospitals, Queen's Medical Centre (Nottingham, UK). The study was registered in Clinicaltrials.gov (Identifier: NCT02593877).

\section{Patient population and safety}

The ACIT study included adult trauma patients who met the local criteria for full trauma team activation. According to the protocol, patients who received more than $2000 \mathrm{~mL}$ of fluids before arrival in the emergency department (ED) or who arrived more than 2 hours from time of injury were excluded, as were patients who were pregnant, had known liver failure, preexisting bleeding disorders or were taking oral anticoagulants other than aspirin. Initial consent was provided by a physician independent of the study. Written informed consent was obtained as soon as possible from the patients or their next of kin. The study was approved by local ethical authorities and performed in accordance with local ethical regulations and the Declaration of Helsinki.

For ITACTIC the inclusion criteria were similar as ACIT; but restricted to adult trauma patients (according to local definitions) presenting with clinical signs of haemorrhagic shock, according to the responsible trauma team leader, the local MTP was activated, according to the participating institutions' specific routines, and first transfusion was initiated.

Participants had to be randomised within $3 \mathrm{~h}$ of injury and $1 \mathrm{~h}$ of admission to the ED of the participating study site.

Agreement was provided on behalf of incapacitated patients by a personal consultee (PC) or a nominated consultee (NC). A written informed consent was given when the patient regained 
the physical and mental capacity to provide such consent. For included patients who did not regain full capacity, informed consent was given by a PC. The study was approved by local ethical authorities and performed in accordance with local ethical regulations and the Declaration of Helsinki.

Pre-defined interim analysis was performed after the enrolment of 100 patients and a Data Safety Monitoring Board reviewed all data on outcomes for every 50 patients enrolled. Patients included in ITACTIC have a high risk of morbidity and mortality, hence a high risk of adverse events and serious adverse events (SAEs). SAEs, expected or not, were recorded on a SAE form, and reported in the final analyses. SAEs considered to be related to intervention or unexpected, were reported to sponsor within 24 hours and to the main research ethics committee.

\section{Paper 1}

\section{Data collection, sampling techniques and measurements for development of algorithms}

In the ACIT study, patient demographics, time and mechanism of injury, pre-hospital fluid administration, vital signs on admission, total amount of fluids and blood products administered within the first 12 hours, and CCTs on admission were collected prospectively. Injury severity was scored when relevant information was available. TEG and ROTEM values were recorded electronically and transferred manually to the case report form (CRF). ACIT started recruiting patients in 2008 with ROTEM analysis. TEG analyses were added in parallel from 2013. For study 1, data from ACIT patients enrolled from the August 2008 to December 2014 were included. VHA assays selected were the ROTEM assays EXTEM and FIBTEM, and standard TEG, rTEG and FF TEG. The CCTs included were PT, fibrinogen concentration and platelet count. PT was converted to INR in accordance with the specific reagents and device characteristics in the respective laboratories. Fibrinogen was measured by 
the Clauss method [171]. Up to 2014 all analyses were done with TEG5000®

Thrombelastograph Haemostasis Analyzer (Haemonetics Corp., Braintree, MA, US) and ROTEM Delta ${ }^{\circledR}$ (formerly TEM international GmbH, Munich, Germany, now Instrumentation Laboratory, Bedford, MA, USA),

\section{Algorithm development}

Trauma algorithms have to be easy to implement and follow in a stressful situation.

Parameters have to be available early and easy to remember and recognize for the clinicians. All trauma centres in the TACTIC program had empiric massive transfusion strategies [172] with 1:1:1 ratio of blood products following best existing evidence [116] and TXA was administered to all patients when ongoing bleeding was suspected according to guidelines [87]. Our algorithms were constructed to guide additional haemostatic therapy based on the principle of goal-directed personalized therapy.

\section{Definitions}

To identify TEG and ROTEM parameters able to recognize coagulopathic trauma patients in need of additional haemostatic therapy, we had to define trigger values for the different therapies. In absence of any commonly accepted and validated ROTEM and TEG definitions of coagulopathy in trauma, we based the definitions on best existing evidence and international guidelines [74,87,101]. Coagulopathy was defined as INR above 1.2, hypofibrinogenemia was defined as fibrinogen below $2.0 \mathrm{~g} / \mathrm{L}$ and thrombocytopenia was defined as platelet counts below $100 \times 10^{9} / \mathrm{L}$. No accepted laboratory definition of hyperfibrinolysis in trauma patients exists, we therefore had to identify threshold values for treatment of hyperfibrinolysis on TEG and ROTEM, through their relationship with mortality and transfusion requirements. 


\section{Statistical analysis}

Multiple imputations were performed on all TEG and ROTEM data to deal with missing data. Relevant statistical analyses were also performed with non-imputed data, to confirm that multiple imputations had not introduced unacceptable bias. Multiple imputation was not done on the outcome data as definitions of coagulopathy, transfusion data or mortality.

Receiver Operating Characteristic (ROC) curves evaluate and compare predictive models, when the predictive variable is continuous, and the outcome is dichotomous or dichotomised. ROC curves do this by plotting sensitivity (the probability of a positive value to be true positive) against 1 - specificity (the probability of a negative value to be positive). We constructed univariate regression models to identify significant VHA parameters capable of identifying coagulopathy, hypofibrinogenemia and thrombocytopenia, and area under the curves (AUC) were calculated. AUC-values are used to rank the abilities of different variables to predict the outcome. An AUC value of 0.5 indicates that the variable has no discriminative ability, while an AUC of 1.0 has a perfect discriminative ability.

To identify threshold values for our relevant parameters, initially Youden Index $(\mathrm{J}=$ sensitivity + specificity-1) were calculated. Maximum Youden Index is suggested to be the optimal cut-off when sensitivity and specificity is equally important.

However, as the intervention in the algorithms are known to carry acceptable risks in a lifethreatening situation, we decided to accept low specificity. Instead of using Youden Index we decided to calculate threshold values for TEG and ROTEM parameters with sensitivity approaching $60 \%, 70 \%, 80 \%$ and $90 \%$ with corresponding specificity, negative predictive value and positive predictive value. 
Initially statistical analyses were done with both coagulopathy and transfusion requirement as outcomes. We decided to use coagulopathy as target for the algorithms as the aim of our study was to create algorithms to normalize the coagulation.

\section{Algorithms}

Available haemostatic therapy shown to be able to improve trauma resuscitation include fibrinogen concentrate or cryoprecipitate, additional plasma, platelets or TXA. No other haemostatic drugs have as of today, shown any additional beneficial effects on trauma haemorrhage.

Our algorithms were constructed to add these haemostatic products to an empiric, best practice MTP. The algorithms had to be pragmatic, as there are several uncertainties and no complete universal understanding of TIC.

For the algorithms we selected the earliest available parameter, that provided the required information and threshold levels with at least $70 \%$ sensitivity, despite low specificity. We also selected thresholds that were easy to recall and accepted small differences from calculated values (e.g. rounding up or down) if that not significantly alter the performance of the individual parameter. For the therapies and doses associated with these thresholds, we chose best practice levels from current guidelines, even though there are little evidence to support these doses. The chosen parameters had to be corresponding for TEG and ROTEM. For the CCT algorithm we chose the definition parameters. Additional TXA could only be prescribed in the VHA group, because only VHAs can provide specific threshold values for hyperfibrinolysis. 


\section{Paper 2}

Paper 2 is a publication of the ITACTIC protocol. We describe the background, the methods, and the procedures for the RCT (presented in the following section).

\section{Paper 3 ITACTIC}

\section{Randomisation}

Enrolled patients were block randomised per centre to the VHA or the CCT study arm. Randomisation was generated by an independent statistician and performed by sealed envelopes. The trial was unblinded, as blinding was impossible. Study centres were predesigned to conduct either Thromboelastography (TEG®) or Rotational Thromboelastometry (ROTEM®), in order to achieve approximately equal sample size in the TEG and ROTEM cohort. Academic Medical Centre, The Royal London Hospital, Kliniken der Stadt Köln and John Radcliffe Hospital were using ROTEM, while Rigshospitalet, Oslo University Hospital and Nottingham University Hospital were using TEG.

\section{Trial procedures}

Algorithms were developed and published for the purpose of ITACTIC (paper 1). During active haemorrhage, samples were drawn for VHA/ CCT analyses at baseline and after every 4 units of RBC until haemostasis. The results from each blood sample were acted upon as soon as they were available. For the VHA arm, this implied acting upon the parameters as they were appearing and not waiting until the VHA trace was completed. The first sample drawn after an intervention was completed, was used to guide the next intervention. 
For the purpose of this trial, haemostasis and end of study-care, was defined as one hour after the last RBC given when the treating clinician believed primary haemostasis had been achieved.

\section{Outcomes}

The primary outcome of this study was proportion of subjects who, at 24 hours after injury, were alive and free of massive transfusion (defined as 10 or more units of RBCs). Secondary endpoints include all-cause mortality at 6- and 24-hours and 28- and 90-days post admission, outcomes describing coagulopathy (defined as PTr $>1.2$ ), blood product administered, 28days ventilator-free and ICU free days, total hospital length of stay, 28-days symptomatic thromboembolic events, incidence of transfusion related complications and organ dysfunction, and health economic outcomes.

\section{Statistical analysis}

The planned sample size was 392 patients for which MTP was activated and transfusions initiated, 196 in each study arm. Data from the ACIT study estimated that approximately $28 \%$ of patients would need massive transfusion or die. With a decreased proportion to $15 \%$ in the VHA group, a power of $80 \%$ and a two-sided alpha of $0.05,170$ patients per group were required. Assuming a drop-out rate of 15\%, 196 patients were needed per group. The statistical analyses were performed following a statistical analysis plan (SAP) defined according to protocol ahead of the study. Independent statisticians, recruited by the sponsor at Queen Mary University of London, were in charge of the statistical analyses. Primary and secondary outcomes were analysed as intention to treat (ITT) and per protocol (PP). The PP group excluded patients who did not have at least one VHA or CCT test performed, patients who died within 60 minutes after baseline blood sampling and patients 
who achieved haemostasis within 60 minutes of baseline sampling. For patients excluded in the PP analyses, the outcome was believed not to be affected by the study interventions. The pre-defined subgroups were analysed by ITT for primary and secondary outcomes.

The primary endpoint was assessed by logistic regression to produce an odds ratio with $95 \%$ confidence intervals. Mortality was compared between the two arms as a binary outcome by logistic regression and using the Kaplan-Meier method and the log-rank test. Time to haemostasis, total number of blood products, 28-day ventilator-free and ICU-free days, total number of blood products transfused, total hospital length of stay and EQ-5D quality-of-life scores were compared using the Wilcoxon-Mann-Whitney or Chi-square tests. WilcoxonMann-Whitney were chosen since none of the parameters were normally distributed. Post hoc analyses of the secondary outcome of numbers receiving study interventions and time to intervention were performed with Wilcoxon-Mann-Whitney tests.

Primary and secondary outcomes were also analysed for the prespecified subgroups of the ITT population: patients with severe TBI (defined as brain Abbreviated Injury Scale (AIS) 4,5 or 6), patients without severe TBI (defined as brain AIS < 4), patients on prior oral anticoagulant therapy (except for aspirin), patients who received a massive transfusion (10 or more units of RBCs first 24 hours), patients arriving coagulopathic (defined as PTr above 1.2) and patients without coagulopathy at admission (defines as PTr 1.2 or below). Post hoc adjustment for baseline and admission characteristics (age, sex, type of injury, heart rate at admission, systolic blood pressure at admission, GCS at admission and BD at admission) was done for the primary endpoint and 28-day mortality for the TBI subgroup. All applied tests were two-sided and p-values of less than 0.05 were considered statistically significant. Statistical analyses were performed using Stata version 13. 


\section{RESULTS}

\section{Paper 1}

A total of 2287 patients were recruited to ACIT in the inclusion period. ROTEM was performed in 2019 patients, whereas 968 patients had TEG results on admission. Overall, approximately $6,5 \%$ of the population was coagulopathic according to definition, and $15 \%$ had at least 4 units of RBC transfusion first 12 hours.

Corresponding TEG and ROTEM values performed similarly in the univariate regression models. Given the principle of timely available, adequately performing and pragmatic triggers, we chose the earliest available parameter among those parameters with the highest AUCs. For obvious reasons, the same parameter cannot guide different therapies in the same algorithm. ROTEM parameters selected for the algorithms had AUCs between 0.75 and 0.80 . The AUCs for corresponding TEG parameters were slightly lower, between 0.54 and 0.72 . The poorer performance among TEG parameters where probably due to the overall lower number of patients with TEG values in the cohort.

For detection of hypofibrinogenemia we chose the fibrin-specific assays FIBTEM and FF TEG, and the earliest available parameter provided the relevant information were FIBTEM CA at 5 minutes (CA5) and FF TEG MA, as the TEG 5000 assay does not provide amplitude results earlier than the maximum amplitude.

Only $44(2 \%)$ ROTEM patients and $17(1,5 \%)$ TEG patients had low platelet counts, which resulted in low AUCs, especially for TEG parameters. The selected parameters to detect low platelet counts were the subtracting amplitude of FIBTEM CA5 from the EXTEM CA5 and the FF TEG MA from the rTEG MA.

Due to the earlier available results, we selected rTEG rather than Kaolin TEG for all TEG parameters except for hypofibrinogenemia. There was no significant loss in sensitivity or 
specificity using rTEG results. EXTEM was selected as ROTEM parameters except for hypofibrinogenemia for the same reason.

To define threshold values for additional plasma despite sufficient replacement of fibrinogen, we selected the upper reference value from the manufactures for EXTEM CT and r-TEG ACT, as the calculated value for INR above 1.2 for both relevant TEG and ROTEM parameters were within the normal reference range.

Hyperfibrinolysis was identified through examination of relationship with lysis parameters and clinical outcomes. For ROTEM there was a step-change in mortality with a ML above $20 \%$, and a similar mortality outcome was seen with a Li30 of $85 \%$. Only $3.5 \%$ of those with a Li30 above $85 \%$ subsequently developed a ML > 20\%, of which 4 patients died. Mean 24hour RBC requirements also increased markedly at Li30 levels below 85\%. For TEG detection of hyperfibrinolysis, there was an increase in mortality at rTEG Ly30 values above $10 \%$ with an associated increase in $\mathrm{RBC}$ requirements at this same threshold.

We based the choice of doses for the different interventions on current best practice. For fibrinogen supplementation we suggested a replacement dose of $4 \mathrm{~g}$ either as cryoprecipitate or fibrinogen concentrate, for low platelets we suggested an additional pool of platelets transfusion, for low coagulation activity despite normal fibrinogen activity we suggested 4 extra units of plasma (either as Octaplasma ${ }^{\circledR}$ or FFP), and with evidence of persistent fibrinolysis we suggested an additional bolus of $1 \mathrm{~g}$ TXA.

Based on our analyses we constructed algorithms for TEG, ROTEM and CCTs, presented in Figure 1. 


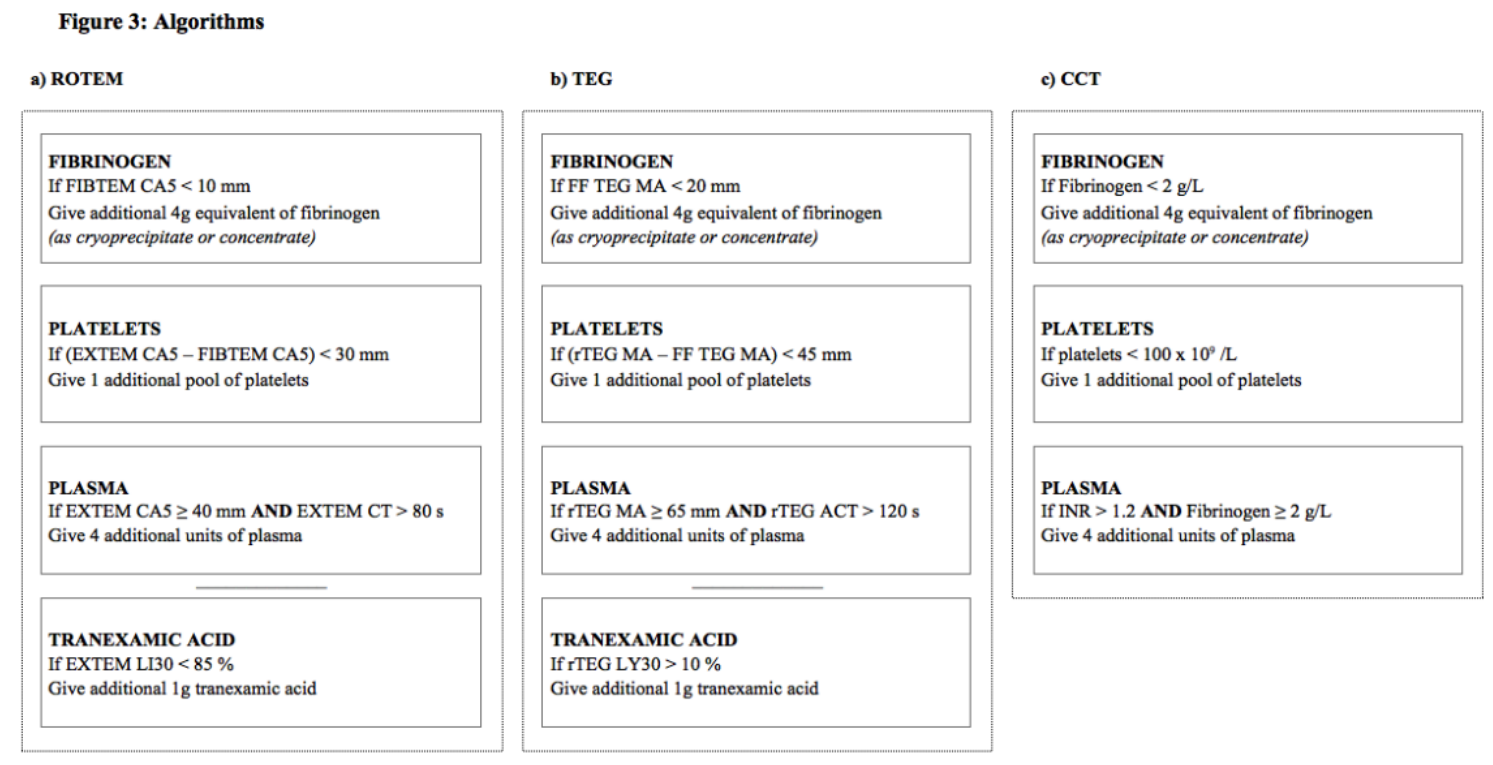

$C A 5=$ clot amplitude at 5 minutes, $C T=$ clotting time, $L I 30=$ lysis Index at 30 minutes, $F F=$ functional fibrinogen, $r T E G=$ rapid $T E G, M A=$ maximum amplitude, $A C T=$ activated clotting time, $L Y 30=$ clot lysis at 30 minutes

\section{Paper 3 ITACTIC}

From $1^{\text {st }}$ of June 2016 to $30^{\text {th }}$ of July 2018, 411 were randomized and 396 patients were included in the intention-to-treat analysis: 195 patients in the CCT group and 201 in the VHA group. The overall population was in accordance with what is expected for severe trauma patients. The majority was male, young to middle-aged and had sustained blunt injury. Baseline demographics and injury and admission characteristics were similar among the two study groups. Median ISS were 26 with interquartile range (IQR) approximately 17-36, whilst a score above 15 indicate severe injury.

102 patients (29\%) were coagulopathic on admission, with an INR above 1.2, and this group was more severely injured than the overall population with a median ISS of 30 (IQR 19-38). 74 patients (19\%) had a concomitant severe brain injury, and this group had a very high injury severity with a median ISS of 38 (IQR 34-50). Among the severe TBI group 31 patients (42\%) were coagulopathic with INR above 1.2. 
There were no differences in the primary endpoint alive and free of massive transfusion at 24 hours between the ITT population and the PP population.

We found no difference in mortality between the two groups in the overall population in the ITT or the PP group. 28-days mortality in the ITT VHA group was $25 \%$ versus $28 \%$ in the CCT group. This pattern persisted to 90 days.

On the other hand, there was a significantly reduced 28-days mortality in the severe TBI subgroup for the VHA arm (44\% vs $74 \%$, OR $0.28,95 \%$ CI $0.10-0.74, p=0.016)$, which persisted at 90 days. This result persisted after adjusting for baseline and admission characteristics (OR $0.1695 \%$ CI 0.03-0.90) (presented in supplementary materials to the article). In addition, in the predefined subgroup of patients who presented with coagulopathy, there was a non-significant trend towards lower mortality in the VHA group, with a mortality at 28 days of $41 \%$ compared to $55 \%$ in the CCT group (OR $0.56,95 \%$ CI:0.26-1.24, p-value 0.070).

Overall, patients in both arms received a median of six units of RBCs administered over the first 24 hours and the proportion of patients receiving a massive transfusion (defined as 10 or more units of RBCs in the first 24 hours) were equal between the two arms (28\% CCT vs $26 \%$ VHA).

254 patients had a normal $\mathrm{PTr}$ (PTr of 1.2 or below) at admission and very few of them developed a prolonged PTr during resuscitation; 9 (7\%) in the VHA group versus $14(11 \%)$ in the CCT group.

There were no differences in critical care outcomes, rate of multiple organ dysfunction, hospital length of stay, ventilator-free days or ICU-free days at day 28. Quality of life (EQ5D) scores was similar in both groups and were similar at both discharge/28 days and 90 days. Safety and the incidence of symptomatic thromboembolic events was the same in both groups, as were also safety profiles and causes of death. 
For the post hoc analyses of patients receiving study intervention, a significantly larger group of VHA patients received a study intervention compared to the CCT group. This difference could be identified both for baseline to haemostasis and injury to 24 hours and included both the ITT and PP population and in all the predefined subgroup except for the coagulopathic subgroup. From injury to haemostasis $36 \%$ of the ITT population received a study intervention in the CCT group compared to $67 \%$ in the VHA group, and $44 \%$ compared to $69 \%$ from injury to 24 hours ( $\mathrm{p}$-value $<0,001$ ). In the TBI group $23 \%$ received a study intervention in the CCT group compared to $70 \%$ in the VHA group from injury to haemostasis and $34 \%$ compared to $74 \%$ from injury to 24 hours (p-value $<0,001)$ (presented in supplementary materials to the article). First study interventions were given earlier after admission and injury in the VHA group than the CCT group. For the ITT population, first intervention was given a median of 19 minutes earlier in the VHA group (VHA: 61 mins (IQR 48-85) vs CCT: 80 mins (IQR 60-106) from baseline, p-value < 0.05). Time to first study intervention from admission and injury was shorter in the VHA group for both ITT and PP and for all subgroups, although the differences were not significant for the severe TBI nor for the coagulopathic group. The subgroup of severe TBI included only 74 patients. Significantly more fibrinogen and platelets were given to the VHA group from baseline to haemostasis, both in the ITT and PP population and in the TBI subgroup. A median of $4 \mathrm{~g}$ fibrinogen (IQR 0-4) was given to the ITT and PP population and the TBI subgroup in the VHA group compared to 0 (IQR 0-4) in the CCT group (p-value < 0.05). 


\section{DISCUSSION}

\section{Trauma resuscitation algorithms}

We have defined accurate, rapidly available parameters identifying coagulation derangements in trauma patients. Threshold values for these parameters were identified to guide additional haemostatic therapy, added to an empiric optimized MTP according to current best evidence and guidelines. Our algorithms are the first and to date, the only corresponding algorithms for both CCT, TEG and ROTEM published for trauma resuscitation, hence applicable for all hospitals admitting trauma patients, regardless of device or method available for monitoring coagulation.

The algorithms were based on data from a large cohort of trauma patients. Little evidence had previously been published on parameters best predicting coagulopathy and guiding haemostatic therapy in trauma. Pragmatically, we chose parameters for the algorithms that were available early, easy to remember and recognizable to clinicians to make them easy to follow in a stressful situation.

Simultaneously with our algorithms, a couple of other data driven VHA algorithms for guiding trauma resuscitation were published. In 2017, the Denver group published an algorithm for r-TEG [173] based on data from 190 trauma patients and the aim was to predict the need for massive transfusion, unlike our algorithms that were constructed to correct coagulopathy. In 2018, the same Denver group published a data-driven algorithm for Kaolin TEG, based on more than 800 patients and with the same aim as for the r-TEG algorithm above [174]. These algorithms deviate both in parameters and thresholds from ours, and they are limited to one VHA device and no other tests. During the developing process of our algorithms, outcome measures for the algorithms were discussed. The three most used outcome measures for defining VHA parameters in trauma resuscitation are prediction of mortality, massive transfusion and/or coagulopathy [157,165]. Based on knowledge of TIC 
and how TIC affects outcomes, we found it reasonable to attempt to identify bleeding patients with coagulopathy when constructing algorithms for guiding haemostatic therapy, instead of massive transfusion which is an endpoint.

Our algorithms have been tested in the RCT ITACTIC. However, further external validation should be performed for the purpose of optimizing the parameters and threshold values for guiding trauma resuscitation.

Although our algorithms were data driven, little or no evidence exists to support the doses. The doses suggested were based on existing guidelines and expert opinions. The selected doses had a generally acceptable risk in a life-threatening setting, but further research is required to define optimal interventions.

\section{VHA vs CCTs}

Our results from ITACTIC showed that for mild to moderate injury, it does not matter how you monitor resuscitation, as long as the resuscitation strategy adheres to best existing evidence, coagulation is monitored, and the protocol is followed. The standard empiric transfusion delivered was balanced during haemorrhage across both study arms and included early TXA. Study interventions were given significantly earlier and more frequently in the VHA arm, but according to our results, this difference in timeline or frequencies for additional therapy did not impact on outcomes for patients without coagulation deficiencies or TBI.

Only approximately $30 \%$ of the patients were coagulopathic at admission in line with previously published data [21] on severely injured populations. Coagulopathy was defined as INR above 1.2, but patients in the VHA cohort received significantly more study interventions than in the CCT cohort. This might reflect undetected coagulopathy due to lack 
of sensitivity of INR, which is a limitation of the study. However, in our study, this did not affect the clinical outcomes in the overall population.

Despite no overall effect of VHA monitoring on the primary outcome, when patients were coagulopathic or with combined bleeding and severe TBI, closer monitoring of coagulation with VHA, improved mortality, although the results were only significant for the TBI group. These subgroups were small, and the results might have been a chance finding. However, patients with coagulopathy or TBI had high injury severity and high mortality compared to the overall group, and rapidly available results from coagulation tests with VHA devices may impact on survival for these most severely injured patients. Therefore, it might be reasonable to recommend VHA monitoring in haemorrhaging trauma patients, whenever severe TBI or coagulopathy is suspected on admission. As it is impossible to rule out TBI without a CT scan, which is often delayed to after haemorrhage control, close coagulation monitoring with VHA might be beneficial to all severely injured and bleeding patients.

Previously, only one RCT, performed by Gonzales et al, has compared the effect of a VHAguided resuscitation strategy with resuscitation guided by CCTs [175]. This study was stopped after inclusion of 111 (TEG 56, CCT 55) patients due to significantly higher 28 days survival in the TEG group in an intention-to-treat analysis; 20 deaths $(36,4 \%)$ in the CCT group vs. 11 in the VHA -group (19,6\%). However, patients in the CCT-group did not get adequate haemostatic therapy on admission due to the study design. Patients were permitted to have only 4 units of RBCs and 2 units of FFP administered before results from the CCT or VHA were available. Further RBCs were transfused while bleeding was ongoing to maintain a haemoglobin of at least $10 \mathrm{~g} / \mathrm{dL}$, while administration of plasma, cryoprecipitate, platelets and TXA were only triggered by either CCTs or TEG results. The CCT results were available after approximately 30 to 45 minutes, while the TEG results were available within 5 minutes, resulting for the CCT patients in a 30-45 minutes delay after the 2 first units of FFPs to get 
adequate haemostatic therapy. While waiting for haemostatic therapy, patients had crystalloids and RBCs depending on haemoglobin. In addition, even though minimizing crystalloids is worldwide accepted as an important part of DCR to reduce haemodilution and coagulopathy, both groups had a median of approximately $3800 \mathrm{ml}$ of crystalloids within 2 hours from injury and more than 6000ml within 4 hours from injury. Large amounts of crystalloids in both arms and inadequate haemostatic therapy in the CCT arm, leads us to question the results from this study. In comparison, all patients in ITACTIC were managed following a massive transfusion strategy with a 1:1:1 ratio of RBC:plasma:platelets administered empirically, TXA was given, crystalloids were minimized, and whenever test results were available, additional haemostatic therapy was delivered if required. The delayed time to CCTs results is similar in ITACTIC and the trial by Gonzales et al, but this delay is deemed less important for outcome for minor to moderately injured bleeding patients, when first-line empiric therapy is balanced.

Interestingly, ITACTIC showed a tendency towards increased 28-days mortality in the noncoagulopathic group using VHA (OR 1.49, 95\% CI 0.75-2.93, p-value 0.07). This group had significantly more platelets and fibrinogen from baseline to haemostasis, than the CCT group. Whether some of this pro-coagulant therapy was unnecessary in a non-coagulopathic population and potentially worsening outcome is unclear. The thresholds in our algorithms were constructed for haemostatic therapy in coagulopathic patients. This might have resulted in overtreatment of normo- and hyper-coagulable patients. More specifically, we suspect the subtracted amplitude threshold for administration of additional platelets might have resulted in unnecessary platelet transfusions This has to be explored further. 


\section{TXA, fibrinolysis and brain injury}

In ITACTIC, patients had $1 \mathrm{~g}$ of TXA as a bolus followed by $1 \mathrm{~g}$ infusion over 8 hours as part of the massive transfusion strategy. This strategy is according to guidelines [87,101] and in line with the CRASH-2 protocol [126]. However, although empiric TXA is widely included in existing MTPs, the use of TXA is still controversial and debated in trauma.

The trauma community in the United States is sceptical to the results from the CRASH-2 study, as most of the patients were included in middle- and low-income countries with limited resources and where the health care systems differed widely from the United States [176,177]. This scepticism is maintained by studies showing no effect of empirically administered TXA. In a study from Harvin and colleagues in 2015 on 1000 patients, TXA was not associated with any improvement in mortality. However, the study was retrospective, the group receiving TXA were more severely injured and there were no differences in adverse events [178]. In addition, recent studies have suggested increased risk of thromboembolisms after TXA. In a military study from 2017, TXA was an independent risk factor for thromboembolism [179]. This paper also described underuse of TXA, as $26 \%$ of the patient receiving massive transfusion failed to get TXA, but also an overuse of TXA in $6 \%$ of the patients. In 2018, a retrospective propensity matched study from Pittsburgh showed no significant survival benefits of TXA and an increased risk of thromboembolism [180]. This being said, a recent meta-analysis from 2018 of more than 40.000 patients with post-partum or trauma haemorrhage (from CRASH-2 [126] and WOMAN trial [181] ), found that vascular occlusive events did not increase with TXA, regardless of timing [182].

The scepticism to empiric administration of TXA is also associated with the fibrinolytic shutdown theory $[67,69]$ described in the introduction. Supporters of this theory are concerned that TXA is associated with a persistent fibrinolytic shutdown and a risk factor for 
increased mortality due to microvascular thrombosis leading to organ failure [67]. In 2018, Meizoso and colleagues showed increased risk of fibrinolytic shutdown after TXA, measured on admission to the Intensive Care Unit (ICU). The same group published in 2016 a study of trauma patients in ICU, showing that 34 of 78 patients with fibrinolytic shutdown on admission to ICU had persisting fibrinolytic shutdown after 1 week. This persisting fibrinolytic shutdown after trauma was independently associated with increased mortality. Concerns about increased mortality when TXA is given to patients with physiological levels of fibrinolysis has also been suggested by Moore and colleagues [183]. The evidence for TXA-guided therapy, even when hyperfibrinolysis is measured by VHA remains limited. A post hoc analysis of PROPPR including patients with hyperfibrinolysis on TEG at admission, showed increased 6 hours survival with TXA, but did not increase long term outcomes in severely injured trauma patients [184].

However, critics of the shutdown theory, argue that patients who might benefit from early administration of TXA, miss out or get delayed treatment, due to waiting times for the lysis results from the VHA $[176,185,186]$. The meta-analysis of patients with post-partum and trauma haemorrhage (from CRASH-2 [126] and WOMAN trial [181] ) showed a 10\% reduction in survival benefit for every 15 minutes of treatment delay until 3 hours, after which there was no benefit [182].

These conflicting views on fibrinolysis and TXA in trauma call for a randomized controlled trial addressing the fibrinolytic system with targeted treatment strategies. In addition, according to the fibrinolytic shutdown theory, in the future, identifying persistently reduced fibrinolytic activity 24 hours after injury may be a target for treatment strategies to avoid thromboembolism following hypofibrinolysis [67]. 
Both the dose and the complete mechanism of the effect of TXA in trauma remain incompletely understood [176]. TXA might have additional roles to its anti-fibrinolytic effect, in inflammation, tissue remodelling and in endothelial cells, that might be beneficial to trauma patients. Recently, an in vitro study of the effects of TXA on endothelial cells exposed to stress, showed protective effects against endothelial damage when administered within 60 minutes after injury [187].

The current evidence for TXA shows no effect and poorer outcome, when administered later than 3 hours after injury $[126,176,182]$. This negative effect of late TXA is still not understood. Locke and colleagues have suggested a theory of TXA binding to uPA resulting in late excessive delocalized plasmin in blood and plasma which digests fibrinogen. As uPA levels increase later than tPA with a peak at 8 hours, this might result in late bleeding [186]. Ongoing and future research will hopefully give a better understanding of the role of TXA in trauma resuscitation.

TBI is responsible for at least $25 \%$ of deaths in critically bleeding patients, and $50 \%$ of all trauma deaths [8]. TBI and coagulopathy have been associated with progressive intracranial haemorrhage and mortality $[188,189]$. Although Samuels and colleagues suggest that severe TBI is not associated with fibrinolysis abnormalities [190], hyperfibrinolysis is widely accepted as an important factor for the coagulopathy in TBI, even though the exact mechanism remains unclear [59]. Severe TBI has often been excluded from clinical trials of trauma haemorrhage which has led to lack of evidence for TXA in TBI. However, the last couple of years, some studies looking at isolated TBI and TXA have been published, admittedly with conflicting results. Late 2019, a large civilian RCT (CRASH-3) published positive results regarding TXA in TBI patients [191]. CRASH-3 was a randomised, placebocontrolled trial from 29 countries including more than 12000 TBI patients. Patients were 
eligible if they had a Glasgow Coma Score (GCS) of 12 or lower or intracranial bleeding on CT scan, and no major extracranial bleeding. The results showed no statistically significant difference in the primary outcome of TBI-related death, whether patients with GCS 3 or bilateral unreactive pupils were excluded or not. However, the results demonstrated a reduced risk for TBI-related deaths with TXA in the subgroup of patients with mild-to-moderate TBI (GCS 9 to 15), but not in the group of patients with severe TBI (GCS 3-8). Early treatment was also more effective than late treatment in the mild to moderate TBI group, while no time effect was seen in the severe TBI group. The risk reduction of TBI related deaths was greatest within the first 24 hours after injury, as anticipated by the authors. The risk of venous thromboembolism and seizures were similar between the groups. CRASH-3 is met by the same criticism as CRASH-2. A large part of the patients was enrolled in low- and middleincome countries, and the transfer value to modern treatment in high-income countries, is debated. The neurosurgical facilities in a large proportion of the study sites were limited, which might have affected the results, especially for the severe TBI patients. In addition, the primary endpoint was defined as TBI-related deaths which is based on a clinical decision made by the treating doctors which is a biased outcome. The reduced mortality was also only found in a subgroup and hence, one could argue that it should only be "hypothesis generating" for future research and not evidence for clinical decisions.

A retrospective review of trauma patients with TBI and massive transfusion in the military operation in Iraq and Afghanistan, was also published in 2019 [192]. TXA was independently associated with improved outcomes compared to a propensity matched cohort treated without TXA, and the study was unable to find any increased risk of thromboembolism in the TXA cohort. However, in 2020, a multicentre randomized double-blinded controlled trial from North America including 966 patients showed no difference in 6-month neurologic outcome, 28 day mortality or progress of intracranial haemorrhage between patients with moderate to 
severe TBI (defined as prehospital GCS $<13$ ) who received either a $1 \mathrm{~g}$ prehospital TXA bolus followed by a $1 \mathrm{~g}$ inhospital TXA infusion or a $2 \mathrm{~g}$ prehospital TXA bolus followed by an inhospital placebo infusion compared with participants who received a prehospital placebo bolus followed by an inhospital placebo infusion [193].

Efficacy and safety of TXA in TBI patients with any type of intracranial haemorrhage was studied in a systematic review and meta-analysis published very recently [194]. Nine RCTs were included and no effect of TXA on all-cause mortality, disability or increased risk of adverse events like thromboembolism or seizure were found. Since CRASH-3 constituted the majority of the patients in this meta-analysis, the authors performed a sensitivity analysis excluding CRASH-3, but this did not change the results or conclusion.

To complicate and confuse this even more, Bossers and colleagues published in December 2020 results from a retrospective analysis of data from a prospective observational multicentre study from Netherland [195]. In this study of patients with suspected severe TBI $(\mathrm{GCS}<8)$ treated by one of the Dutch physician-staffed helicopter emergency medical service, administration of TXA was associated with significantly increased mortality in a group of patients with isolated TBI.

In this context, ITACTIC represents unexpected but important, additional knowledge, providing an opportunity for improving outcomes in trauma care. ITACTIC shows a real mortality reduction of direct coagulation management for the bleeding trauma patients with TBI. Despite all patients receiving TXA as part of an empiric transfusion strategy, avoiding delayed antifibrinolytic therapy, close coagulation monitoring with VHA showed significantly improved 28-days survival. This results from ITACTIC have to be followed by further research on combined and isolated TBI. Moreover, the mechanisms by which correction of coagulopathy reduces mortality from TBI is unclear and represents an important area for future research. 


\section{Best practice management of trauma haemorrhage in the future}

Despite different theories of the mechanisms behind ATC and the ongoing debate about hyperfibrinolysis versus fibrinolytic shutdown, a widespread agreement exists throughout the trauma community worldwide, about current best practice trauma resuscitation. Leading trauma research networks have recently published reviews describing the same resuscitation strategies $[32,34,94,99,102,196]$. This strategy includes DCR with haemostatic resuscitation including a balanced ratio of blood products, followed by individualization according to coagulation monitoring. Currently accepted additional haemostatic therapy includes plasma, platelets, fibrinogen supplementation and TXA.

However, in Austria, the trauma community has for more than a decade applied a factor-based resuscitation strategy. They advocate RBCs to keep haemoglobin above $10 \mathrm{~g} / \mathrm{L}$, and administer fibrinogen and prothrombin complex concentrates as first line therapy, according to ROTEM traces, instead of plasma [144]. Less utilisation of blood products with theoretically less transfusion reactions, are suggested as benefits from this strategy [170,197,198]. In 2011, this Austrian group reported reduced MOF following the concentratestrategy in a very small cohort of matched patients from two retrospective data registries [198]. However, more recent evidence supporting this strategy is limited. In 2017, the Austrian group presented a single centre RCT using ROTEM-guided thresholds to compare resuscitation with FFP versus factor concentrate (fibrinogen concentrate and prothrombin complex concentrate (PCC)), looking at MOF as the primary outcome [199]. 94 patients were included (50 PCC; 44 FFP) of whom 7 died. This study was terminated early due to futility and safety concerns. A high proportion of patients in the FFP cohort needed massive transfusion and rescue therapy with fibrinogen concentrate, compared with the factor concentrate cohort. 23 of 44 in the FFP cohort had rescue therapy with mainly fibrinogen concentrate. The authors concluded that first line factor concentrate was superior to FFP. 
However, the data presented hardly support this conclusion. Treatment was initiated in the factor concentrate arm after a median of 10 minutes versus patients in the FFP group who had first treatment after a median of 50,5 minutes. As expected, following the delayed therapy in the FFP group, the time to correction of coagulopathy and normalisation of bleeding was prolonged. It is reasonable to believe that the time to treatment have affected the transfusion requirements. Hence, it seems appropriate to argue that this study compared early versus late haemostatic treatment rather than factor concentrate versus FFP. At present, the use of PCC and fibrinogen concentrate instead of plasma in trauma resuscitation is mostly limited to Austria. However, it seems likely that PCC will find its place as additional haemostatic therapy in trauma resuscitation in the future.

\section{The future of trauma resuscitation}

Despite a dramatic increase in knowledge about trauma resuscitation and the pathophysiology of TIC over the last two decades, many questions remain unanswered. We might have achieved deeper insight into the trauma physiology, but with deeper insight, new complex questions have been identified.

We still don't have a complete understanding of the pathophysiology or definition of TIC. TIC can clinically appear as a spectrum from hypercoagulability to hypocoagulability or hyperfibrinolysis, and the process is dynamic. Better understanding of the molecular mechanisms, including the types of cells, factors and mediators involved at different stages, as well as the dynamics and timing of the different stages in this process, is needed to improve treatment strategies directly targeting the coagulation.

The use of VHA in trauma has increased over the last decade, despite lack of evidence. As trauma resuscitation is stressful, even simple algorithms might be too complex. There is a risk 
that increasing use of VHA in trauma, might cause overuse and unnecessary treatment of test results in patients with no or limited bleeding.

This thesis is part of the ongoing research to improve evidence for individualization of trauma resuscitation. However, choice of parameters, threshold values, interventions and dosing have to be further explored. We still don't know for sure what patients will benefit most from VHA driven treatment and how to define when targeted coagulation monitoring is indicated. VHAs analyse whole blood and are not able to consider the effect of the endothelium on the coagulation. We know that the endothelium is important both in the pro-coagulant and the anti-coagulant process, as described in the introduction. A target for future research would be coagulation monitoring device able to map the endothelial function.

Most commonly, the empiric MTPs consist of blood components. However, over the last years, fresh whole blood (FWB) has been reintroduced in the debate about trauma resuscitation [122]. Based on military experience, FWB is increasingly available in the prehospital and hospital settings, but we still lack firm evidence in the civilian setting for the benefits of FWB versus risk of adverse events and the logistical and production challenges, especially regarding wastage.

Approximately 50\% of all trauma deaths are from TBI. The understanding of coagulation deficiencies in isolated TBIs remains limited, and we still lack knowledge about optimal resuscitation. It is widely accepted that coagulopathy is common among TBI with progressive haemorrhage[188]. CRASH-3 suggests TXA to all moderate TBIs [191], but the results from CRASH-3 are debated and the recent meta-analysis including the CRASH-3 data on TXA to isolated TBI showed no effect of TXA on mortality or disability [194]. Our study has shown promising results with rapidly available VHA results in guiding treatment in haemorrhaging 
patients with severe TBI. However, if and how VHA will be part of future care in isolated TBI, must be explored further.

As resuscitation has improved, we have seen a new entity of MOF. However, we lack evidence to understand why some patients, after successful resuscitation, progress into an irreversible MOF, with high mortality. Historically, most MOF deaths were caused by ARDS, kidney failure or ACS. However, these MOF entities have almost disappeared following better resuscitation and ICU therapy [8,11]. Currently, MOF deaths after trauma are more often due to a massive vascular and cardiac failure $[8,11]$. We need a better understanding of the pathophysiology of this MOF, and how to prevent it. This is an important area of current and future trauma research. The Copenhagen group explores endotheliopathy in different cohorts of shocked patients. Prostacyclin is known to have cytoprotective effects in addition to vasodilation and platelet inhibition [200]. The Copenhagen group has demonstrated improved endothelial function and integrity after low dose Prostacyclin, measured by validated biomarkers, and no adverse effects on blood pressure or platelet functions in pilot studies on acute coronary stent patients [201], in major surgery [202] and in septic shock [203]. In septic shock patients, lower sequential organ failure assessment (SOFA) score, reduced time on ventilator and lower 30- and 90-day mortality were also demonstrated. This knowledge has resulted in an ongoing multicentre RCT (SHINE-TRAUMA, ClinicalTrials.gov Identifier: NCT03903939) comparing 72-hours infusion of low dose prostacyclin compared to placebo, in bleeding trauma patients, looking at length of ICU stay, reflecting the need for organ support, but also mortality and outcomes regarding therapy for MOF.

Another approach to future improvement in MOF might be looking at plasma transfusions in the post resuscitation phase. Preclinical studies using rat and porcine models of haemorrhagic 
shock reveal that resuscitation with plasma decreases the proinflammatory state, and reduces vascular permeability and lung inflammation through stabilization of the endothelial glycocalyx when compared with artificial colloid, albumin, and lactated Ringer [40]. Haemorrhaging trauma patients often require high volumes to restore circulation post resuscitation. Today, crystalloids are worldwide used in this phase, despite knowledge of negative effects of crystalloids regarding coagulation, endothelial and vascular functions. Future research to address volume therapy in the post resuscitation phase, is necessary and awaited.

Improvement in trauma resuscitation has led to increased survival throughout the resuscitation phase. We need to focus on improvement in intensive care treatment and innovations to identify and prevent the new entity of trauma MOF, and hopefully we can continue to improve survival in the future.

\section{Limitations/ methodological considerations}

\section{Paper 1:}

We have described the need for external validation of the algorithms. Patients were enrolled at 6 different trauma centres with slightly different MTPs and treatment strategies that may have affected the results. In addition, there were significant differences in the number of patients included at the different centres. ROTEM and TEG results were not blinded to clinicians in all centres which may have affected the treatment, but probably not the results of the admission blood samples used for the definition of coagulation deficiencies.

Even though the algorithms were constructed based on a large cohort of trauma patients, the number of patients with coagulation deficiencies and transfusion requirement were limited. Only approximately $6 \%$ were coagulopathic on admission and $15 \%$ received more than 4 units of RBC during the first 12 hours. As the TEG cohort was about half the size of the 
ROTEM cohort, the low number of patients with coagulopathy and transfusion requirements especially affected the TEG parameters and threshold values more. In addition, low platelet count is rare in TIC, and the number of patients with low platelet counts was low, which affected the accuracy of the parameters and threshold values selected for platelet transfusion. Moreover, the association between the platelet number and platelet function in trauma is also uncertain $[79,81]$, and the ability to monitor platelet function with VHA in trauma is unclear [81].

Thresholds for hyperfibrinolysis had to be determined based on the relationship with transfusion requirement and mortality in the absence of a definitive laboratory comparator test. The prehospital use of TXA might affected the numbers of patients presenting with hyperfibrinolysis on admission but should not affect the threshold values selected for prolonged hyperfibrinolysis.

Furthermore, the algorithms are based on definitions following current best evidence guidelines for coagulopathy in trauma patients. However, INR is unprecise and might misdiagnose the coagulopathy. Future research will hopefully make us able to identify both coagulopathy and platelet dysfunction more precisely.

Finally, as described previously, little or no evidence exists to support the chosen doses for the interventions. The doses suggested were based on existing guidelines and expert opinions and need further assessment.

\section{Paper 3:}

ITACTIC was a pragmatic international multicentre study. Although standard of care was relative similar in all participating centres [172] and algorithms were standardized and optimized, there were still differences in trauma care between the study sites, potentially 
affecting the results. However, these differences reflect a real-world situation and affected both arms equally due to block randomization.

The number of patients enrolled in the various study sites varied, also potentially affecting the results. The power calculations were based upon the ACIT cohort, as the only evidence base available to estimate effects. However, the group of haemorrhaging trauma patients in ACIT was small. The uncertainties in the power calculation resulted in a degree of underpowering of the trial regarding outcomes in the subgroups, especially the coagulopathic patients.

The study was resource demanding with very tight follow up in extremely stressful situations. This resulted in a substantially number of missing data, most importantly for PTr, used to identify coagulopathic patients. It also prevented us from being able to fully assess the ability of the algorithms to correct coagulation deficits.

Retrospectively, we have also recognised several challenges with the electronic CRF. Text fields made the statistical analyses challenging, especially regarding study interventions. This was further complicated by the analyses having to be done by independent statisticians without clinical knowledge. For future RCTs, less text fields in the CRF are recommended.

\section{Ethical considerations}

Conducting clinical studies in trauma is challenging, with important safety considerations and needs concerning the priority for delivering life-saving patient care.

ACIT is a prospective observational study and involves no interventions, while ITACTIC compared different ways of monitoring and adjusting resuscitation. However, in ITACTIC, there was no evidence to suggest one arm to be superior to the other.

Most of the patients enrolled in ACIT and ITACTIC were incapacitated at the time of enrolment and necessary research related intervention, preventing us from providing informed consent/agreement from the patient, relative or other PC. Consent for initial enrolment in the 
trials was therefore sought from a NC, in the form of independent clinicians who were familiar with these studies and consenting process and were present at the enrolment of the patient. If, and when the patients regained the physical and mental capacity to provide consent, information was given to them and written informed consent was obtained. For any patient who did not regain full capacity, agreement was sought from a relative or other appropriate PC. If the patient or PC declined to give consent/agreement for enrolment, his/her wishes were respected.

Patients who are incapable of providing consent in emergency situations constitute an established exception to the general rule of informed consent in clinical trials. Both ACIT and ITACTIC followed the Mental Capacity Act (England; 2005) and the Declaration of Helsinki (2013) and were approved by all Research Ethics Committees (RECs) for the participating study sites.

To prevent survival bias, the ITACTIC study included all deaths. In cases where the subject died before consent/agreement from the patient or the PC was obtained, the patient was planned to remain in the trial based on the signed declaration obtained by the NC. A consent from PC was not acquired, in order to minimise stress and anxiety associated with the unexpected and traumatic death of a relative. This was accepted by all RECs, except for the Norwegian. To avoid possible survival bias following this, efforts were made to reach relatives to all patients who died at Oslo University Hospital, even though we experienced this to be unpleasant for the relatives. Finally, only 2 patients who died were excluded because lack of contact with the relatives. Excluding these 2 patients resulted in minor survival bias in the ITACTIC study. However, survival bias will be a challenge to all emergency research in Norway, as long as the RECs require consent from all patients or their PCs. This has been and will further be addressed to the Regional Ethics Committees in Norway. 


\section{CONCLUSION}

We have constructed goal-directed personalized resuscitation algorithms for haemorrhaging trauma patients. These algorithms include an empiric resuscitation strategy based on best existing evidence, individualized with haemostatic therapy added according to results from coagulation monitoring during the resuscitation. Our algorithms are data-driven, based on prospectively collected data from a large cohort of trauma patients and are the first and to date, the only corresponding algorithms for both CCT, TEG and ROTEM published for trauma resuscitation, hence applicable for all hospitals admitting trauma patients, regardless of device or method available for monitoring coagulation.

The RCT ITACTIC compared the algorithms for VHAs to CCT in guiding goal-directed individualized resuscitation in trauma haemorrhage. We did not find any effect on mortality or need for massive transfusion between the two algorithms. However, patients monitored with VHA underwent more haemostatic therapy and delivered earlier after admission compared to patients in the CCT group, without affecting the outcomes. Hence, our results indicate that for the overall trauma patient, the different monitoring methods do not affect outcomes, as long as the resuscitation strategy adheres to best existing evidence, coagulation is monitored, and the protocol is followed.

However, VHA guided algorithms showed a mortality benefit for the most severely injured patients with coagulopathy and/or severe TBI. These subgroups were small, and the results might be a chance finding, but still, while waiting for further studies, it might be reasonable to recommend VHA monitoring in haemorrhaging trauma patients, when severe TBI or coagulopathy is suspected on admission. Closer and less time-consuming coagulation monitoring with VHAs might be beneficial to these patients. Since coagulopathy and severe TBI are difficult to rule out in the initial resuscitation phase, it might be reasonable to 
recommend close coagulation monitoring with VHA to all severe haemorrhaging trauma patients. 


\section{REFERENCES}

1. Baksaas-Aasen K, Dieren SV, Balvers K, et al (2019) Data-driven Development of ROTEM and TEG Algorithms for the Management of Trauma Hemorrhage. Ann Surg 270:1178-1185. https://doi.org/10.1097/sla.0000000000002825

2. Baksaas-Aasen K, Gall L, Eaglestone S, et al (2017) iTACTIC - implementing Treatment Algorithms for the Correction of Trauma-Induced Coagulopathy: study protocol for a multicentre, randomised controlled trial. Trials 18:486. https://doi.org/10.1186/s13063-0172224-9

3. Baksaas-Aasen K, Gall LS, Stensballe J, et al (2021) Viscoelastic haemostatic assay augmented protocols for major trauma haemorrhage (ITACTIC): a randomized, controlled trial. Intens Care Med 47:49-59. https://doi.org/10.1007/s00134-020-06266-1

4. WHO WHO: Injuries and Violence - The facts 2014. https://apps.who.int/iris/bitstream/handle/10665/149798/9789241508018_eng.pdf;jsessionid= 21418C550670E2E91371D5C2480061AF?sequence=1. Accessed 18 Nov 2020

5. Folkehelseinstituttet Dødsårsakregisteret 2018. http://statistikkbank.fhi.no/dar/. Accessed 18 Nov 2020

6. Koh EY, Oyeniyi BT, Fox EE, et al (2018) Trends in Potentially Preventable Trauma Deaths Between 2005-2006 and 2012-2013. Am J Surg 218:501-506.

https://doi.org/10.1016/j.amjsurg.2018.12.022

7. Stanworth SJ, Davenport R, Curry N, et al (2016) Mortality from trauma haemorrhage and opportunities for improvement in transfusion practice. Brit J Surg 103:357-365.

https://doi.org/10.1002/bjs.10052

8. Cole E, Weaver A, Gall L, et al (2019) A Decade of Damage Control Resuscitation: New Transfusion Practice, New Survivors, New Directions. Ann Surg Publish Ahead of Print:1-6. https://doi.org/10.1097/sla.0000000000003657

9. Marsden M, Carden R, Navaratne L, et al (2018) Outcomes following trauma laparotomy for hypotensive trauma patients. J Trauma Acute Care 85:620-625.

https://doi.org/10.1097/ta.0000000000001988

10. Harvin JA, Maxim T, Inaba K, et al (2017) Mortality after emergent trauma laparotomy. J Trauma Acute Care 83:464-468. https://doi.org/10.1097/ta.0000000000001619

11. Brohi K, Gruen RL, Holcomb JB (2019) Why are bleeding trauma patients still dying? Intens Care Med 45:709-711. https://doi.org/10.1007/s00134-019-05560-X

12. Oyeniyi BT, Fox EE, Scerbo M, et al (2017) Trends in 1029 trauma deaths at a level 1 trauma center: Impact of a bleeding control bundle of care. Inj 48:5-12.

https://doi.org/10.1016/j.injury.2016.10.037 
13. Bardes JM, Inaba K, Schellenberg M, et al (2018) The contemporary timing of trauma deaths. J Trauma Acute Care 84:893-899. https://doi.org/10.1097/ta.0000000000001882

14. Furie B, Furie BC (2008) Mechanisms of Thrombus Formation. New Engl J Medicine 359:938-949. https://doi.org/10.1056/nejmra0801082

15. Hoffman M, Monroe DM (2001) A cell-based model of hemostasis. Thromb Haemostasis $85: 958-65$

16. Giordano S, Spiezia L, Campello E, Simioni P (2017) The current understanding of trauma-induced coagulopathy (TIC): a focused review on pathophysiology. Intern Emerg Med 12:981-991. https://doi.org/10.1007/s11739-017-1674-0

17. Dobson GP, Letson HL, Sharma R, et al (2015) Mechanisms of early trauma-induced coagulopathy. J Trauma Acute Care 79:301-309.

https://doi.org/10.1097/ta.0000000000000729

18. Cohen MJ, Christie SA (2016) New understandings of post injury coagulation and resuscitation. Int J Surg 33:242-245. https://doi.org/10.1016/j.ijsu.2016.05.037

19. Duchesne JC, Kimonis K, Marr AB, et al (2010) Damage Control Resuscitation in Combination With Damage Control Laparotomy; A Survival Advantage. J Trauma Inj Infect Critical Care 69:46-52. https://doi.org/10.1097/ta.0b013e3181df91fa

20. Moore EE (1996) Staged laparotomy for the hypothermia, acidosis, and coagulopathy syndrome. The American Journal of Surgery 172:405-410.

https://doi.org/https://doi.org/10.1016/S0002-9610(96)00216-4

21. Brohi K, Singh J, Heron M, Coats TJ (2003) Acute Traumatic Coagulopathy. The Journal of Trauma: Injury, Infection, and Critical Care 54:1127-1130.

https://doi.org/10.1097/01.ta.0000069184.82147.06

22. MacLeod JBA, Lynn M, McKenney MG, et al (2003) Early Coagulopathy Predicts Mortality in Trauma. J Trauma Inj Infect Critical Care 55:39-44.

https://doi.org/10.1097/01.ta.0000075338.21177.ef

23. Hess JR, Brohi K, Dutton RP, et al (2008) The Coagulopathy of Trauma; A Review of Mechanisms. J Trauma Inj Infect Critical Care 65:748-754.

https://doi.org/10.1097/ta.0b013e3181877a9c

24. Brohi K, Cohen MJ, Davenport RA (2007) Acute coagulopathy of trauma; mechanism, identification and effect. Curr Opin Crit Care 13:680-685.

https://doi.org/10.1097/mcc.0b013e3282f1e78f

25. Noel P, Cashen S, Patel B (2013) Trauma-Induced Coagulopathy: From Biology to Therapy. Semin Hematol 50:259-269. https://doi.org/10.1053/j.seminhematol.2013.06.009

26. Maegele M, Spinella PC, Schöchl H (2012) The Acute Coagulopathy of Trauma. Shock 38:450-458. https://doi.org/10.1097/shk.0b013e31826dbd23 
27. Frith D, Brohi K (2012) The pathophysiology of trauma-induced coagulopathy. Curr Opin Crit Care 18:631-636. https://doi.org/10.1097/mcc.0b013e3283599ab9

28. Cardenas JC, Wade CE, Holcomb JB (2014) Mechanisms of trauma-induced coagulopathy. Curr Opin Hematol 21:404-409.

https://doi.org/10.1097/moh.0000000000000063

29. Cap A, Hunt B (2014) Acute traumatic coagulopathy. Curr Opin Crit Care 20:638-645. https://doi.org/10.1097/mcc.0000000000000158

30. Davenport RA, Brohi K (2016) Cause of trauma-induced coagulopathy. Curr Opin Anaesthesiol 29:212-219. https://doi.org/10.1097/aco.0000000000000295

31. Simmons JW, Powell MF (2016) Acute traumatic coagulopathy: pathophysiology and resuscitation. Brit J Anaesth 117:iii31-iii43. https://doi.org/10.1093/bja/aew328

32. Cohen MJ, Christie SA (2017) Coagulopathy of Trauma. Crit Care Clin 33:101-118. https://doi.org/10.1016/j.ccc.2016.08.003

33. Meledeo MA, Herzig MC, Bynum JA, et al (2017) Acute traumatic coagulopathy:The elephant in a room of blind scientists. J Trauma Acute Care 82:S33-S40.

https://doi.org/10.1097/ta.0000000000001431

34. Harris T, Davenport R, Mak M, Brohi K (2018) The Evolving Science of Trauma

Resuscitation. Emerg Med Clin N Am 36:85-106. https://doi.org/10.1016/j.emc.2017.08.009

35. Blaine KP, Steurer MP (2019) Viscoelastic Monitoring to Guide the Correction of Perioperative Coagulopathy and Massive Transfusion in Patients with Life-Threatening Hemorrhage. Anesthesiol Clin 37:51-66. https://doi.org/10.1016/j.anclin.2018.09.004

36. Brohi K, Cohen MJ, Ganter MT, et al (2007) Acute Traumatic Coagulopathy; Initiated by Hypoperfusion. Modulated Through the Protein C Pathway? Ann Surg 245:812-818. https://doi.org/10.1097/01.sla.0000256862.79374.31

37. Johansson PI, Henriksen HH, Stensballe J, et al (2017) Traumatic Endotheliopathy. Ann Surg 265:597-603. https://doi.org/10.1097/sla.0000000000001751

38. Gando S (2009) Acute Coagulopathy of Trauma Shock and Coagulopathy of Trauma; A Rebuttal. You Are Now Going Down the Wrong Path. J Trauma Inj Infect Critical Care 67:381-383. https://doi.org/10.1097/ta.0b013e3181a84f63

39. Moore HB, Gando S, Iba T, et al (2019) Defining trauma-induced coagulopathy with respect to future implications for patient management: Communication from the SSC of the ISTH. J Thromb Haemost 18:740-747. https://doi.org/10.1111/jth.14690

40. Watson JJJ, Pati S, Schreiber MA (2016) Plasma Transfusion. Shock 46:468-479. https://doi.org/10.1097/shk.0000000000000663 
41. Johansson PI, Stensballe J, Rasmussen LS, Ostrowski SR (2011) A High Admission Syndecan-1 Level, A Marker of Endothelial Glycocalyx Degradation, Is Associated With Inflammation, Protein C Depletion, Fibrinolysis, and Increased Mortality in Trauma Patients. Ann Surg 254:194-200. https://doi.org/10.1097/sla.0b013e318226113d

42. Johansson PI, Ostrowski SR (2010) Acute coagulopathy of trauma: Balancing progressive catecholamine induced endothelial activation and damage by fluid phase anticoagulation. Med Hypotheses 75:564-567. https://doi.org/10.1016/j.mehy.2010.07.031

43. Ostrowski SR, Johansson PI (2012) Endothelial glycocalyx degradation induces endogenous heparinization in patients with severe injury and early traumatic coagulopathy. $\mathrm{J}$ Trauma Acute Care 73:60-66. https://doi.org/10.1097/ta.0b013e31825b5c10

44. Ostrowski SR, Henriksen HH, Stensballe J, et al (2017) Sympathoadrenal activation and endotheliopathy are drivers of hypocoagulability and hyperfibrinolysis in trauma. J Trauma Acute Care 82:293-301. https://doi.org/10.1097/ta.0000000000001304

45. Johansson PI, Stensballe J, Rasmussen LS, Ostrowski SR (2012) High circulating adrenaline levels at admission predict increased mortality after trauma. J Trauma Acute Care Surg 72:428-436. https://doi.org/10.1097/ta.0b013e31821e0f93

46. Johansson P, Stensballe J, Ostrowski S (2017) Shock induced endotheliopathy (SHINE) in acute critical illness - a unifying pathophysiologic mechanism. Crit Care 21:25. https://doi.org/10.1186/s13054-017-1605-5

47. Brohi K, Cohen MJ, Ganter MT, et al (2008) Acute Coagulopathy of Trauma; Hypoperfusion Induces Systemic Anticoagulation and Hyperfibrinolysis. J Trauma Inj Infect Critical Care 64:1211-1217. https://doi.org/10.1097/ta.0b013e318169cd3c

48. Davenport RA, Guerreiro M, Frith D, et al (2017) Activated Protein C Drives the Hyperfibrinolysis of Acute Traumatic Coagulopathy. Anesthesiology 126:115-127. https://doi.org/10.1097/aln.0000000000001428>

49. Cohen MJ, Call M, Nelson M, et al (2012) Critical Role of Activated Protein C in Early Coagulopathy and Later Organ Failure, Infection and Death in Trauma Patients. Ann Surg 255:379-385. https://doi.org/10.1097/sla.0b013e318235d9e6

50. Howard BM, Kornblith LZ, Cheung CK, et al (2016) Inducing Acute Traumatic Coagulopathy In Vitro: The Effects of Activated Protein C on Healthy Human Whole Blood. Plos One 11:e0150930. https://doi.org/10.1371/journal.pone.0150930

51. Zyl N van, Milford EM, Diab S, et al (2016) Activation of the protein C pathway and endothelial glycocalyx shedding is associated with coagulopathy in an ovine model of trauma and hemorrhage. J Trauma Acute Care 81:674-684.

https://doi.org/10.1097/ta.0000000000001190

52. Gangloff C, Mingant F, Theron M, et al (2019) New considerations on pathways involved in acute traumatic coagulopathy: the thrombin generation paradox. World J Emerg Surg 14:57. https://doi.org/10.1186/s13017-019-0276-8 
53. Gando S, Mayumi T, Ukai T (2018) Activated protein C plays no major roles in the inhibition of coagulation or increased fibrinolysis in acute coagulopathy of trauma-shock: a systematic review. Thrombosis J 16:13. https://doi.org/10.1186/s12959-018-0167-3

54. Chapin JC, Hajjar KA (2015) Fibrinolysis and the control of blood coagulation. Blood Rev 29:17-24. https://doi.org/10.1016/j.blre.2014.09.003

55. Gall L, Brohi K, Davenport R (2017) Diagnosis and Treatment of Hyperfibrinolysis in Trauma (A European Perspective). Semin Thromb Hemost 43:224-234. https://doi.org/10.1055/s-0036-1598001

56. Hunt BJ, Segal H (1996) Hyperfibrinolysis. J Clin Pathol 49:958. https://doi.org/10.1136/jcp.49.12.958

57. Kauvar DS, Lefering R, Wade CE (2006) Impact of Hemorrhage on Trauma Outcome; An Overview of Epidemiology, Clinical Presentations, and Therapeutic Considerations. J Trauma Inj Infect Critical Care 60:S3-S11. https://doi.org/10.1097/01.ta.0000199961.02677.19

58. Raza I, Davenport R, Rourke C, et al (2013) The incidence and magnitude of fibrinolytic activation in trauma patients. J Thromb Haemost 11:307-314.

https://doi.org/10.1111/jth.12078

59. Gall LS, Davenport RA (2018) Fibrinolysis and antifibrinolytic treatment in the trauma patient. Curr Opin Anaesthesiol 31:227-233. https://doi.org/10.1097/aco.0000000000000561

60. Whyte C, Mitchell J, Mutch N (2017) Platelet-Mediated Modulation of Fibrinolysis. Semin Thromb Hemost 43:115-128. https://doi.org/10.1055/s-0036-1597283

61. Vulliamy P, Gillespie S, Gall LS, et al (2017) Platelet transfusions reduce fibrinolysis but do not restore platelet function during trauma hemorrhage. J Trauma Acute Care 83:388-397. https://doi.org/10.1097/ta.0000000000001520

62. Chapman MP, Moore EE, Moore HB, et al (2016) Overwhelming tPA release, not PAI-1 degradation, is responsible for hyperfibrinolysis in severely injured trauma patients. J Trauma Acute Care 80:16-25. https://doi.org/10.1097/ta.0000000000000885

63. Gando S, Sawamura A, Hayakawa M (2011) Trauma, Shock, and Disseminated Intravascular Coagulation. Ann Surg 254:10-19.

https://doi.org/10.1097/sla.0b013e31821221b1

64. Gando S, Wada H, Thachil J, (ISTH) TS and SC on D of the IS on T and H (2013)

Differentiating disseminated intravascular coagulation (DIC) with the fibrinolytic phenotype from coagulopathy of trauma and acute coagulopathy of trauma-shock (COT/ACOTS). J Thromb Haemost 11:826-835. https://doi.org/10.1111/jth.12190

65. Otomo Y, Gando S Local hemostasis, immunothrombosis, and systemic disseminated intravascular coagulation in trauma and traumatic shock. Critical Care 19:72.

https://doi.org/DOI 10.1186/s13054-015-0735-x 
66. Chakrabarti R, Hocking ED, Fearnley GR (1969) Reaction pattern to three stresseselectroplexy, surgery, and myocardial infarction — of fibrinolysis and plasma fibrinogen. $\mathrm{J}$ Clin Pathol 22:659. https://doi.org/10.1136/jcp.22.6.659

67. Moore HB, Moore EE, Neal MD, et al (2019) Fibrinolysis Shutdown in Trauma: Historical Review and Clinical Implications. Anesthesia Analgesia 129:762-773. https://doi.org/10.1213/ane.0000000000004234

68. Moore HB, Moore EE, Gonzalez E, et al (2014) Hyperfibrinolysis, physiologic fibrinolysis, and fibrinolysis shutdown. J Trauma Acute Care 77:811-817. https://doi.org/10.1097/ta.0000000000000341

69. Moore HB, Moore EE, Liras IN, et al (2016) Acute Fibrinolysis Shutdown after Injury Occurs Frequently and Increases Mortality: A Multicenter Evaluation of 2,540 Severely Injured Patients. J Am Coll Surgeons 222:347-355.

https://doi.org/10.1016/j.jamcollsurg.2016.01.006

70. Moore EE, Moore HB, Gonzalez E, et al (2016) Rationale for the selective administration of tranexamic acid to inhibit fibrinolysis in the severely injured patient. Transfusion 56:S110 S114. https://doi.org/10.1111/trf.13486

71. Gall LS, Vulliamy P, Gillespie S, et al (2018) The S100A10 Pathway Mediates an Occult Hyperfibrinolytic Subtype in Trauma Patients. Ann Surg 269:1184-1191. https://doi.org/10.1097/sla.0000000000002733

72. Hagemo JS, Stanworth S, Juffermans NP, et al (2014) Prevalence, predictors and outcome of hypofibrinogenaemia in trauma: a multicentre observational study. Crit Care 18:R52. https://doi.org/10.1186/cc13798

73. Rourke C, Curry N, Khan S, et al (2012) Fibrinogen levels during trauma hemorrhage, response to replacement therapy, and association with patient outcomes. J Thromb Haemost 10:1342-1351. https://doi.org/10.1111/j.1538-7836.2012.04752.x

74. Frith D, Goslings JC, Gaarder C, et al (2010) Definition and drivers of acute traumatic coagulopathy: clinical and experimental investigations. J Thromb Haemost 8:1919-1925. https://doi.org/10.1111/j.1538-7836.2010.03945.x

75. Spahn D, Spahn G, Stein P (2016) Indications and Risks of Fibrinogen in Surgery and Trauma. Seminars Thrombosis Hemostasis 42:147-154. https://doi.org/10.1055/s-00351564841

76. Davenport R, Brohi K (2013) Fibrinogen depletion in trauma: early, easy to estimate and central totrauma-induced coagulopathy. Crit Care 17:190. https://doi.org/10.1186/cc13021

77. Schlimp CJ, Voelckel W, Inaba K, et al (2013) Estimation of plasma fibrinogen levels based on hemoglobin, base excess and Injury Severity Score upon emergency room admission. Crit Care 17:R137. https://doi.org/10.1186/cc12816

78. Martini WZ (2007) The effects of hypothermia on fibrinogen metabolism and coagulation function in swine. Metabolis 56:214-221. https://doi.org/10.1016/j.metabol.2006.09.015 
79. Kutcher ME, Redick BJ, McCreery RC, et al (2012) Characterization of platelet dysfunction after trauma. J Trauma Acute Care 73:13-19.

https://doi.org/10.1097/ta.0b013e318256deab

80. Ramsey MT, Fabian TC, Shahan CP, et al (2016) A prospective study of platelet function in trauma patients. J Trauma Acute Care 80:726-733.

https://doi.org/10.1097/ta.0000000000001017

81. Vulliamy P, Kornblith LZ, Kutcher ME, et al (2020) Alterations in platelet behavior after major trauma: adaptive or maladaptive? Platelets 00:1-10.

https://doi.org/10.1080/09537104.2020.1718633

82. Ireland S, Endacott R, Cameron P, et al (2011) The incidence and significance of accidental hypothermia in major trauma-A prospective observational study. Resuscitation 82:300-306. https://doi.org/10.1016/j.resuscitation.2010.10.016

83. Weuster M, Brück A, Lippross S, et al (2016) Epidemiology of accidental hypothermia in polytrauma patients. J Trauma Acute Care 81:905-912.

https://doi.org/10.1097/ta.0000000000001220

84. Martini WZ, Dubick MA, Pusateri AE, et al (2006) Does Bicarbonate Correct

Coagulation Function Impaired by Acidosis in Swine? J Trauma Inj Infect Critical Care

61:99-106. https://doi.org/10.1097/01.ta.0000215574.99093.22

85. Maegele M, Lefering R, Yucel N, et al (2007) Early coagulopathy in multiple injury: An analysis from the German Trauma Registry on 8724 patients. Inj 38:298-304.

https://doi.org/10.1016/j.injury.2006.10.003

86. Sumislawski JJ, Kornblith LZ, Conroy AS, et al (2018) Dynamic Coagulability after Injury: Is Delaying Venous Thromboembolism Chemoprophylaxis Worth the Wait? J Trauma Acute Care 85:907-914. https://doi.org/10.1097/ta.0000000000002048

87. Rossaint R, Bouillon B, Cerny V, et al (2016) The European guideline on management of major bleeding and coagulopathy following trauma: fourth edition. Crit Care 20:100.

https://doi.org/10.1186/s13054-016-1265-X

88. Cohen MJ (2012) Towards Hemostatic Resuscitation The Changing Understanding of Acute Traumatic Biology, Massive Bleeding, and Damage-Control Resuscitation. Surg Clin N Am 92:877-891. https://doi.org/10.1016/j.suc.2012.06.001

89. Cantle PM, Cotton BA (2017) Balanced Resuscitation in Trauma Management. Surg Clin N Am 97:999-1014. https://doi.org/10.1016/j.suc.2017.06.002

90. Trauma AC of SC on TQaC of SC on (2012) Advanced trauma life support: ATLS : student course manual. American College of Surgeons

91. STONE HH, STROM PR, Mullins RJ (1983) Management of the Major Coagulopathy with Onset during Laparotomy. Ann Surg 197:532-535. https://doi.org/10.1097/00000658198305000-00005 
92. Rotondo MF, Schwab CW, McGonigal MD, et al (1993) Damage control: An approach for improved survival in exsanguinating penetrating abdominal injury.". J Trauma Inj Infect Critical Care 35:375-383. https://doi.org/10.1097/00005373-199207000-00079

93. Rotondo MF, Zonies DH (1997) The Damage Control Sequence Aand Underlying Logic. Surg Clin N Am 77:761-777. https://doi.org/10.1016/s0039-6109(05)70582-X

94. Shapiro MB, Jenkins DH, Schwab CW, Rotondo and MF (2000) Damage Control:

Collective Review. J Trauma Inj Infect Critical Care 49:969-978.

https://doi.org/10.1097/00005373-200011000-00033

95. Cap AP, Pidcoke HF, Spinella P, et al (2018) Damage Control Resuscitation. Mil Med 183:36-43. https://doi.org/10.1093/milmed/usy112

96. Lamb CM, MacGoey P, Navarro AP, Brooks AJ (2014) Damage control surgery in the era of damage control resuscitation. Brit J Anaesth 113:242-249.

https://doi.org/10.1093/bja/aeu233

97. Holcomb JB, Jenkins D, Rhee P, et al (2007) Damage Control Resuscitation; Directly Addressing the Early Coagulopathy of Trauma. J Trauma Inj Infect Critical Care 62:307-310. https://doi.org/10.1097/ta.0b013e3180324124

98. Ball C (2014) Damage control resuscitation: history, theory and technique. Can J Surg 57:55-60. https://doi.org/10.1503/cjs.020312

99. Curry N, Brohi K (2019) Surgery in Traumatic Injury and Perioperative Considerations. Semin Thromb Hemost 46:073-082. https://doi.org/10.1055/s-0039-1697932

100. Tonglet ML (2016) Early Prediction of Ongoing Hemorrhage in Severe Trauma: Presentation of the Existing Scoring Systems. Archives Trauma Res 5:e33377. https://doi.org/10.5812/atr.33377

101. Overview | Major trauma: assessment and initial management | Guidance | NICE. https://www.nice.org.uk/guidance/ng39. Accessed 18 Nov 2020

102. Stensballe J, Ostrowski SR, Johansson PI (2016) Haemostatic resuscitation in trauma: the next generation. Curr Opin Crit Care 22:591-597.

https://doi.org/10.1097/mcc.0000000000000359

103. Bickell WH, Wall MJ, Pepe PE, et al (1995) Immediate versus Delayed Fluid Resuscitation for Hypotensive Patients with Penetrating Torso Injuries. New Engl J Medicine 331:1105-1109. https://doi.org/10.1056/nejm199410273311701

104. Mapstone J, Roberts I, Evans P (2003) Fluid Resuscitation Strategies; A Systematic Review of Animal Trials. J Trauma Inj Infect Critical Care 55:571-589.

https://doi.org/10.1097/01.ta.0000062968.69867.6f

105. Dutton RP, Mackenzie CF, Scalea TM (2002) Hypotensive Resuscitation during Active Hemorrhage: Impact on In-Hospital Mortality. J Trauma Inj Infect Critical Care 52:11411146. https://doi.org/10.1097/00005373-200206000-00020 
106. Tran A, Yates J, Lau A, et al (2018) Permissive hypotension versus conventional resuscitation strategies in adult trauma patients with hemorrhagic shock. J Trauma Acute Care 84:802-808. https://doi.org/10.1097/ta.0000000000001816

107. Malone DL, Hess JR, Fingerhut A (2006) Massive Transfusion Practices Around the Globe and a Suggestion for a Common Massive Transfusion Protocol. J Trauma Inj Infect Critical Care 60:S91-S96. https://doi.org/10.1097/01.ta.0000199549.80731.e6

108. Holcomb JB, Wade CE, Michalek JE, et al (2008) Increased Plasma and Platelet to Red Blood Cell Ratios Improves Outcome in 466 Massively Transfused Civilian Trauma Patients. Transactions Meet Am Surg Assoc 126:97-108.

https://doi.org/10.1097/sla.0b013e318185a9ad

109. Holcomb JB, Junco DJ del, Fox EE, et al (2013) The Prospective, Observational, Multicenter, Major Trauma Transfusion (PROMMTT) Study: Comparative Effectiveness of a Time-Varying Treatment With Competing Risks. Jama Surg 148:127-136.

https://doi.org/10.1001/2013.jamasurg.387

110. Zink KA, Sambasivan CN, Holcomb JB, et al (2009) A high ratio of plasma and platelets to packed red blood cells in the first 6 hours of massive transfusion improves outcomes in a large multicenter study. Am J Surg 197:565-570.

https://doi.org/10.1016/j.amjsurg.2008.12.014

111. Johansson PI, Oliveri RS, Ostrowski SR (2012) Hemostatic resuscitation with plasma and platelets in trauma. J Emergencies Trauma Shock 5:120-125.

https://doi.org/10.4103/0974-2700.96479

112. Borgman MA, Spinella PC, Perkins JG, et al (2007) The Ratio of Blood Products Transfused Affects Mortality in Patients Receiving Massive Transfusions at a Combat Support Hospital. J Trauma Inj Infect Critical Care 63:805-813.

https://doi.org/10.1097/ta.0b013e3181271ba3

113. Maegele M, Lefering R, Paffrath T, et al (2008) Red blood cell to plasma ratios transfused during massive transfusion are associated with mortality in severe multiply injury: a retrospective analysis from the Trauma Registry of the Deutsche Gesellschaft für Unfallchirurgie. Vox Sang 95:112-119. https://doi.org/10.1111/j.1423-0410.2008.01074.x

114. Johansson PI, Stensballe J (2009) REVIEWS: Hemostatic resuscitation for massive bleeding: the paradigm of plasma and platelets - a review of the current literature. Transfusion 50:701-710. https://doi.org/10.1111/j.1537-2995.2009.02458.x

115. Johansson PI, Hansen MB, Sørensen H (2005) Transfusion practice in massively bleeding patients: time for a change? Vox Sang 89:92-96. https://doi.org/10.1111/j.14230410.2005.00668.x

116. Holcomb JB, Tilley BC, Baraniuk S, et al (2015) Transfusion of Plasma, Platelets, and Red Blood Cells in a 1:1:1 vs a 1:1:2 Ratio and Mortality in Patients With Severe Trauma: The PROPPR Randomized Clinical Trial. Jama 313:471-482.

https://doi.org/10.1001/jama.2015.12 
117. Khan S, Davenport R, Raza I, et al (2015) Damage control resuscitation using blood component therapy in standard doses has a limited effect on coagulopathy during trauma hemorrhage. Intens Care Med 41:239-247. https://doi.org/10.1007/s00134-014-3584-1

118. Pati S, Matijevic N, Doursout M-F, et al (2010) Protective Effects of Fresh Frozen Plasma on Vascular Endothelial Permeability, Coagulation, and Resuscitation After Hemorrhagic Shock Are Time Dependent and Diminish Between Days 0 and 5 After Thaw. J Trauma Inj Infect Critical Care 69:S55-S63. https://doi.org/10.1097/ta.0b013e3181e453d4

119. Balvers K, Dieren S van, Baksaas-Aasen K, et al (2017) Combined effect of therapeutic strategies for bleeding injury on early survival, transfusion needs and correction of coagulopathy. Brit J Surg 104:222-229. https://doi.org/10.1002/bjs.10330

120. Sperry JL, Guyette FX, Brown JB, et al (2018) Prehospital Plasma during Air Medical Transport in Trauma Patients at Risk for Hemorrhagic Shock. New Engl J Med 379:315-326. https://doi.org/10.1056/nejmoa1802345

121. Hernandez MC, Thiels CA, Aho JM, et al (2017) Prehospital plasma resuscitation associated with improved neurologic outcomes after traumatic brain injury. J Trauma Acute Care 83:398-405. https://doi.org/10.1097/ta.0000000000001581

122. Chipman AM, Jenne C, Wu F, Kozar RA (2020) Contemporary resuscitation of hemorrhagic shock: What will the future hold? Am J Surg 220:580-588.

https://doi.org/10.1016/j.amjsurg.2020.05.008

123. Stensballe J, Ulrich AG, Nilsson JC, et al (2018) Resuscitation of Endotheliopathy and Bleeding in Thoracic Aortic Dissections. Anesthesia Analgesia 127:920-927. https://doi.org/10.1213/ane.0000000000003545

124. Cardenas JC, Zhang X, Fox EE, et al (2018) Platelet transfusions improve hemostasis and survival in a substudy of the prospective, randomized PROPPR trial. Blood Adv 2:16961704. https://doi.org/10.1182/bloodadvances.2018017699

125. Roberts I (2015) Tranexamic acid in trauma: how should we use it? J Thromb Haemost 13:S195-S199. https://doi.org/10.1111/jth.12878

126. collaborators C-2 trial (2010) Effects of tranexamic acid on death, vascular occlusive events, and blood transfusion in trauma patients with significant haemorrhage (CRASH-2): a randomised, placebo-controlled trial. Lancet 376:23-32. https://doi.org/10.1016/s01406736(10)60835-5

127. Roberts I, Prieto-Merino D, Manno D (2014) Mechanism of action of tranexamic acid in bleeding trauma patients: an exploratory analysis of data from the CRASH-2 trial. Crit Care 18:685. https://doi.org/10.1186/s13054-014-0685-8

128. Roberts I, Perel P, Prieto-Merino D, et al (2012) Effect of tranexamic acid on mortality in patients with traumatic bleeding: prespecified analysis of data from randomised controlled trial. Bmj Br Medical J 345:e5839. https://doi.org/10.1136/bmj.e5839 
129. collaborators C-2, Roberts I, Shakur H, et al (2011) The importance of early treatment with tranexamic acid in bleeding trauma patients: an exploratory analysis of the CRASH-2 randomised controlled trial. Lancet 377:1096-1101.e2. https://doi.org/10.1016/s01406736(11)60278-x

130. Morrison JJ, Dubose JJ, Rasmussen TE, Midwinter MJ (2012) Military Application of Tranexamic Acid in Trauma Emergency Resuscitation (MATTERs) Study. Arch Surgchicago 147:113-119. https://doi.org/10.1001/archsurg.2011.287

131. Morrison JJ, Ross JD, Dubose JJ, et al (2013) Association of Cryoprecipitate and Tranexamic Acid With Improved Survival Following Wartime Injury: Findings From the MATTERs II Study. Jama Surg 148:218-225. https://doi.org/10.1001/jamasurg.2013.764

132. Cole E, Davenport R, Willett K, Brohi K (2015) Tranexamic Acid Use in Severely Injured Civilian Patients and the Effects on Outcomes. Ann Surg 261:390-394. https://doi.org/10.1097/sla.0000000000000717

133. Ker K, Roberts I, Shakur H, Coats TJ (2015) Antifibrinolytic drugs for acute traumatic injury. Cochrane Db Syst Rev 5:CD004896.

https://doi.org/10.1002/14651858.cd004896.pub4

134. Guyette FX, Brown JB, Zenati MS, et al (2020) Tranexamic Acid During Prehospital Transport in Patients at Risk for Hemorrhage After Injury. Jama Surg 156:1-10. https://doi.org/10.1001/jamasurg.2020.4350

135. Johansson PI, Stensballe J, Oliveri R, et al (2014) How I treat patients with massive hemorrhage. Blood 124:3052-3058. https://doi.org/10.1182/blood-2014-05-575340

136. Nakamura Y, Ishikura H, Kushimoto S, et al (2017) Fibrinogen level on admission is a predictor for massive transfusion in patients with severe blunt trauma: Analyses of a retrospective multicentre observational study. Inj 48:674-679.

https://doi.org/10.1016/j.injury.2017.01.031

137. McQuilten ZK, Wood EM, Bailey M, et al (2017) Fibrinogen is an independent predictor of mortality in major trauma patients: A five-year statewide cohort study. Inj 48:1074-1081. https://doi.org/10.1016/j.injury.2016.11.021

138. Stinger HK, Spinella PC, Perkins JG, et al (2008) The Ratio of Fibrinogen to Red Cells Transfused Affects Survival in Casualties Receiving Massive Transfusions at an Army Combat Support Hospital. J Trauma Inj Infect Critical Care 64:S79-S85. https://doi.org/10.1097/ta.0b013e318160a57b

139. Khan S, Brohi K, Chana M, et al (2014) Hemostatic resuscitation is neither hemostatic nor resuscitative in trauma hemorrhage. J Trauma Acute Care 76:561-568.

https://doi.org/10.1097/ta.0000000000000146

140. CRYOSTAT-2. https://www.nhsbt.nhs.uk/clinical-trials-unit/current-trials-andstudies/cryostat-2/. Accessed 18 Nov 2020 
141. Okerberg CK, Williams LA, Kilgore ML, et al (2016) Cryoprecipitate AHF vs. fibrinogen concentrates for fibrinogen replacement in acquired bleeding patients - an economic evaluation. Vox Sang 111:292-298. https://doi.org/10.1111/vox.12417

142. Wong H, Curry N (2018) Do we need cryoprecipitate in the era of fibrinogen concentrate and other specific factor replacement options? Isbt Sci Series 13:23-28.

https://doi.org/10.1111/voxs.12376

143. Winearls J, Campbell D, Hurn C, et al (2017) Fibrinogen in traumatic haemorrhage: A narrative review. Inj 48:230-242. https://doi.org/10.1016/j.injury.2016.12.012

144. Schöchl H, Nienaber U, Hofer G, et al (2010) Goal-directed coagulation management of major trauma patients using thromboelastometry (ROTEM®)-guided administration of fibrinogen concentrate and prothrombin complex concentrate. Crit Care 14:R55. https://doi.org/10.1186/cc8948

145. Schlimp CJ, Ponschab M, Voelckel W, et al (2016) Fibrinogen levels in trauma patients during the first seven days after fibrinogen concentrate therapy: a retrospective study. Scand J Trauma Resusc Emerg Medicine 24:29. https://doi.org/10.1186/s13049-016-0221-8

146. Curry N, Rourke C, Davenport R, et al (2015) Early cryoprecipitate for major haemorrhage in trauma: a randomised controlled feasibility trial. Bja Br J Anaesth 115:76-83. https://doi.org/10.1093/bja/aev134

147. Curry N, Foley C, Wong H, et al (2018) Early fibrinogen concentrate therapy for major haemorrhage in trauma (E-FIT 1): results from a UK multi-centre, randomised, double blind, placebo-controlled pilot trial. Crit Care 22:164. https://doi.org/10.1186/s13054-018-2086-X

148. Nascimento B, Callum J, Tien H, et al (2016) Fibrinogen in the initial resuscitation of severe trauma (FiiRST): a randomized feasibility trial. Brit J Anaesth 117:775-782. https://doi.org/10.1093/bja/aew343

149. Peltan ID, Vusse LKV, Maier RV, Watkins TR (2015) An International Normalized Ratio-Based Definition of Acute Traumatic Coagulopathy Is Associated With Mortality, Venous Thromboembolism, and Multiple Organ Failure After Injury. Crit Care Med 43:1429-1438. https://doi.org/10.1097/ccm.0000000000000981

150. Davenport R, Manson J, De'Ath H, et al (2011) Functional definition and characterization of acute traumatic coagulopathy. Crit Care Med 39:2652-2658. https://doi.org/10.1097/ccm.0b013e3182281af5

151. Goodman MD, Makley AT, Hanseman DJ, et al (2015) All the bang without the bucks. J Trauma Acute Care 79:117-124. https://doi.org/10.1097/ta.0000000000000691

152. Mistral T, Boué Y, Bosson J-L, et al (2017) Performance of point-of-care international normalized ratio measurement to diagnose trauma-induced coagulopathy. Scand J Trauma Resusc Emerg Medicine 25:59. https://doi.org/10.1186/s13049-017-0404-y 
153. Wikkelsø A, Wetterslev J, Møller AM, Afshari A (2016) Thromboelastography (TEG) or thromboelastometry (ROTEM) to monitor haemostatic treatment versus usual care in adults or children with bleeding. Cochrane Db Syst Rev CD007871.

https://doi.org/10.1002/14651858.cd007871.pub3

154. Kaufmann CR, Dwyer KM, Crews JD, et al (1997) Usefulness of Thrombelastography in Assessment of Trauma Patient Coagulation. J Trauma Inj Infect Critical Care 42:716-722. https://doi.org/10.1097/00005373-199704000-00023

155. Ganter MT, Hofer CK (2008) Coagulation Monitoring; Current Techniques and Clinical Use of Viscoelastic Point-of-Care Coagulation Devices. Anesthesia Analgesia 106:13661375. https://doi.org/10.1213/ane.0b013e318168b367

156. Johansson PI, Stissing T, Bochsen L, Ostrowski SR (2009) Thrombelastography and tromboelastometry in assessing coagulopathy in trauma. Scand J Trauma Resusc Emerg Medicine 17:45. https://doi.org/10.1186/1757-7241-17-45

157. Curry NS, Davenport R, Pavord S, et al (2018) The use of viscoelastic haemostatic assays in the management of major bleeding. Brit J Haematol 182:789-806.

https://doi.org/10.1111/bjh.15524

158. Hans GA, Besser MW (2016) The place of viscoelastic testing in clinical practice. Brit J Haematol 173:37-48. https://doi.org/10.1111/bjh.13930

159. Theusinger OM, Wanner GA, Emmert MY, et al (2011) Hyperfibrinolysis Diagnosed by Rotational Thromboelastometry (ROTEM®) Is Associated with Higher Mortality in Patients with Severe Trauma. Anesthesia Analgesia 113:1003-1012.

https://doi.org/10.1213/ane.0b013e31822e183f

160. Chapman MP, Moore EE, Ramos CR, et al (2013) Fibrinolysis greater than 3\&percnt; is the critical value for initiation of antifibrinolytic therapy. J Trauma Acute Care 75:961-967. https://doi.org/10.1097/ta.0b013e3182aa9c9f

161. Whiting P, Al M, Westwood M, et al (2015) Viscoelastic point-of-care testing to assist with the diagnosis, management and monitoring of haemostasis: a systematic review and costeffectiveness analysis. Health Technol Asses 19:1-228. https://doi.org/10.3310/hta19580

162. Luz LTD, Nascimento B, Shankarakutty AK, et al (2014) Effect of thromboelastography (TEG®) and rotational thromboelastometry (ROTEM $®$ ) on diagnosis of coagulopathy, transfusion guidance and mortality in trauma: descriptive systematic review. Crit Care 18:518. https://doi.org/10.1186/s13054-014-0518-9

163. Hunt H, Stanworth S, Curry N, et al (2015) Thromboelastography (TEG) and rotational thromboelastometry (ROTEM) for trauma-induced coagulopathy in adult trauma patients with bleeding. Cochrane Db Syst Rev 2:CD010438.

https://doi.org/10.1002/14651858.cd010438.pub2 
164. Fahrendorff M, Oliveri RS, Johansson PI (2017) The use of viscoelastic haemostatic assays in goal-directing treatment with allogeneic blood products - A systematic review and meta-analysis. Scand J Trauma Resusc Emerg Medicine 25:39.

https://doi.org/10.1186/s13049-017-0378-9

165. Veigas PV, Callum J, Rizoli S, et al (2016) A systematic review on the rotational thrombelastometry (ROTEM $®$ ) values for the diagnosis of coagulopathy, prediction and guidance of blood transfusion and prediction of mortality in trauma patients. Scand J Trauma Resusc Emerg Medicine 24:114. https://doi.org/10.1186/s13049-016-0308-2

166. Bugaev N, Como JJ, Golani G, et al (2020) Thromboelastography and Rotational Thromboelastometry in Bleeding Patients with Coagulopathy: Practice Management Guideline from the Eastern Association for the Surgery of Trauma. J Trauma Acute Care Publish Ahead of Print:1-49. https://doi.org/10.1097/ta.0000000000002944

167. Abdelfattah K, Cripps MW (2016) Thromboelastography and Rotational Thromboelastometry use in trauma. Int J Surg 33:196-201. https://doi.org/10.1016/j.ijsu.2015.09.036

168. Inaba K, Rizoli S, Veigas PV, et al (2015) 2014 Consensus conference on viscoelastic test-based transfusion guidelines for early trauma resuscitation. Journal of Trauma and Acute Care Surgery 78:1220-1229. https://doi.org/10.1097/ta.0000000000000657

169. Winearls J, Reade M, Miles H, et al (2016) Targeted Coagulation Management in Severe Trauma. Anesthesia Analgesia 123:910-924. https://doi.org/10.1213/ane.0000000000001516

170. Schöchl H, Maegele M, Solomon C, et al (2012) Early and individualized goal-directed therapy for trauma-induced coagulopathy. Scand J Trauma Resusc Emerg Medicine 20:15. https://doi.org/10.1186/1757-7241-20-15

171. Clauss A (1957) Gerinnungsphysiologische Schnellmethode zur Bestimmung des Fibrinogens. Acta Haematol-basel 17:237-246. https://doi.org/10.1159/000205234

172. Schäfer N, Driessen A, Fröhlich M, et al (2015) Diversity in clinical management and protocols for the treatment of major bleeding trauma patients across European level I Trauma Centres. Scand J Trauma Resusc Emerg Medicine 23:74. https://doi.org/10.1186/s13049-015$0147-6$

173. Einersen PM, Moore EE, Chapman MP, et al (2017) Rapid thrombelastography thresholds for goal-directed resuscitation of patients at risk for massive transfusion. J Trauma Acute Care 82:114-119. https://doi.org/10.1097/ta.0000000000001270

174. Stettler GR, Sumislawski JJ, Moore EE, et al (2018) Citrated kaolin thrombelastography (TEG) thresholds for goal-directed therapy in injured patients receiving massive transfusion. $\mathrm{J}$ Trauma Acute Care 85:734-740. https://doi.org/10.1097/ta.0000000000002037

175. Gonzalez E, Moore EE, Moore HB, et al (2016) Goal-directed Hemostatic Resuscitation of Trauma-induced Coagulopathy. Ann Surg 263:1051-1059.

https://doi.org/10.1097/sla.0000000000001608 
176. Ramirez RJ, Spinella PC, Bochicchio GV (2017) Tranexamic Acid Update in Trauma. Crit Care Clin 33:85-99. https://doi.org/10.1016/j.ccc.2016.08.004

177. Napolitano LM, Cohen MJ, Cotton BA, et al (2013) Tranexamic acid in trauma. J Trauma Acute Care 74:1575-1586. https://doi.org/10.1097/ta.0b013e318292cc54

178. Harvin JA, Peirce CA, Mims MM, et al (2015) The impact of tranexamic acid on mortality in injured patients with hyperfibrinolysis. J Trauma Acute Care 78:905-911. https://doi.org/10.1097/ta.0000000000000612

179. Johnston LR, Rodriguez CJ, Elster EA, Bradley MJ (2018) Evaluation of Military Use of Tranexamic Acid and Associated Thromboembolic Events. Jama Surg 153:169. https://doi.org/10.1001/jamasurg.2017.3821

180. Myers SP, Kutcher ME, Rosengart MR, et al (2018) Tranexamic acid administration is associated with an increased risk of posttraumatic venous thromboembolism. J Trauma Acute Care 86:20-27. https://doi.org/10.1097/ta.0000000000002061

181. Collaborators WT (2017) Effect of early tranexamic acid administration on mortality, hysterectomy, and other morbidities in women with post-partum haemorrhage (WOMAN): an international, randomised, double-blind, placebo-controlled trial. Lancet 389:2105-2116. https://doi.org/10.1016/s0140-6736(17)30638-4

182. Gayet-Ageron A, Prieto-Merino D, Ker K, et al (2018) Effect of treatment delay on the effectiveness and safety of antifibrinolytics in acute severe haemorrhage: a meta-analysis of individual patient-level data from 40138 bleeding patients. Lancet 391:125-132. https://doi.org/10.1016/s0140-6736(17)32455-8

183. Moore HB, Moore EE, Huebner BR, et al (2017) Tranexamic acid is associated with increased mortality in patients with physiological fibrinolysis. J Surg Res 220:438-443. https://doi.org/10.1016/j.jss.2017.04.028

184. Khan M, Jehan F, Bulger EM, et al (2018) Severely injured trauma patients with admission hyperfibrinolysis. J Trauma Acute Care 85:851-857.

https://doi.org/10.1097/ta.0000000000002022

185. Roberts I (2016) Fibrinolytic shutdown: fascinating theory but randomized controlled trial data are needed. Transfusion 56:S115-S118. https://doi.org/10.1111/trf.13490

186. Locke M, Longstaff C (2020) How treatment delay may lead to loss of effectiveness of tranexamic acid. Anz J Surg 90:416-418. https://doi.org/10.1111/ans.15669

187. Diebel LN, Martin JV, Liberati DM (2017) Early tranexamic acid administration ameliorates the endotheliopathy of trauma and shock in an in vitro model. J Trauma Acute Care 82:1080-1086. https://doi.org/10.1097/ta.0000000000001445

188. Yuan Q, Sun Y, Wu X, et al (2016) Coagulopathy in Traumatic Brain Injury and Its Correlation with Progressive Hemorrhagic Injury: A Systematic Review and Meta-Analysis. J Neurotraum 33:1279-1291. https://doi.org/10.1089/neu.2015.4205 
189. Maegele M, Schöchl H, Menovsky T, et al (2017) Coagulopathy and haemorrhagic progression in traumatic brain injury: advances in mechanisms, diagnosis, and management. Lancet Neurology 16:630-647. https://doi.org/10.1016/s1474-4422(17)30197-7

190. Samuels JM, Moore EE, Silliman CC, et al (2018) Severe traumatic brain injury is associated with a unique coagulopathy phenotype. J Trauma Acute Care 86:686-693. https://doi.org/10.1097/ta.0000000000002173

191. collaborators TC-3 trial (2019) Effects of tranexamic acid on death, disability, vascular occlusive events and other morbidities in patients with acute traumatic brain injury (CRASH3): a randomised, placebo-controlled trial. Lancet 394:1713-1723.

https://doi.org/10.1016/s0140-6736(19)32233-0

192. Morte D, Lammers D, Bingham J, et al (2019) Tranexamic acid administration following head trauma in a combat setting: Does tranexamic acid result in improved neurologic outcomes? J Trauma Acute Care 87:125-129. https://doi.org/10.1097/ta.0000000000002269

193. Rowell SE, Meier EN, McKnight B, et al (2020) Effect of Out-of-Hospital Tranexamic Acid vs Placebo on 6-Month Functional Neurologic Outcomes in Patients With Moderate or Severe Traumatic Brain Injury. Jama 324:961-974. https://doi.org/10.1001/jama.2020.8958

194. Lawati KA, Sharif S, Maqbali SA, et al (2020) Efficacy and safety of tranexamic acid in acute traumatic brain injury: a systematic review and meta-analysis of randomized-controlled trials. Intens Care Med 1-14. https://doi.org/10.1007/s00134-020-06279-w

195. Bossers SM, Loer SA, Bloemers FW, et al (2021) Association Between Prehospital Tranexamic Acid Administration and Outcomes of Severe Traumatic Brain Injury. Jama Neurol 78:. https://doi.org/10.1001/jamaneurol.2020.4596

196. Chang R, Holcomb JB (2017) Optimal Fluid Therapy for Traumatic Hemorrhagic Shock. Crit Care Clin 33:15-36. https://doi.org/10.1016/j.ccc.2016.08.007

197. Schöchl H, Nienaber U, Maegele M, et al (2011) Transfusion in trauma: thromboelastometry-guided coagulation factor concentrate-based therapy versus standard fresh frozen plasma-based therapy. Crit Care 15:R83. https://doi.org/10.1186/cc10078

198. Nienaber U, Innerhofer P, Westermann I, et al (2011) The impact of fresh frozen plasma vs coagulation factor concentrates on morbidity and mortality in trauma-associated haemorrhage and massive transfusion. Inj 42:697-701.

https://doi.org/10.1016/j.injury.2010.12.015

199. Innerhofer P, Fries D, Mittermayr M, et al (2017) Articles Reversal of trauma-induced coagulopathy using first-line coagulation factor concentrates or fresh frozen plasma (RETIC): a single-centre, parallel-group, open-label, randomised trial. The Lancet Haematology 4:e258-e271. https://doi.org/10.1016/s2352-3026(17)30077-7

200. Kawabe J, Yuhki K, Okada M, et al (2010) Prostaglandin I 2 Promotes Recruitment of Endothelial Progenitor Cells and Limits Vascular Remodeling. Arteriosclerosis, Thrombosis, and Vascular Biology 30:464-470.

https://doi.org/https://doi.org/10.1161/ATVBAHA.109.193730 
201. Holmvang L, Ostrowski SR, Dridi NP, Johansson P (2012) A single center, open, randomized study investigating the clinical safety and the endothelial modulating effects of a prostacyclin analog in combination with eptifibatide in patients having undergone primary percutaneous coronary intervention (PCI) for ST-segment elevation myocardial infarction. Prostag Oth Lipid M 99:87-95. https://doi.org/10.1016/j.prostaglandins.2012.08.002

202. Johansson PI, Mortensen CR, Nielsen T, et al (2017) The effect of intraoperative and 6-h postoperative intravenous administration of low-dose prostacyclin on the endothelium, hemostasis, and hemodynamics in patients undergoing a pancreaticoduodenoctemy. Eur J Gastroen Hepat 29:400-406. https://doi.org/10.1097/meg.0000000000000800

203. Berthelsen RE, Ostrowski SR, Bestle MH, Johansson PI (2019) Co-administration of iloprost and eptifibatide in septic shock (CO-ILEPSS) - a randomised, controlled, doubleblind investigator-initiated trial investigating safety and efficacy. Crit Care 23:301.

https://doi.org/10.1186/s13054-019-2573-8 


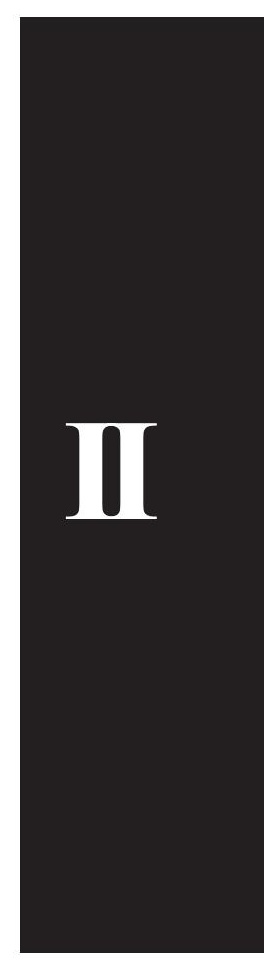





\title{
iTACTIC - implementing Treatment Algorithms for the Correction of Trauma- Induced Coagulopathy: study protocol for a multicentre, randomised controlled trial
}

Kjersti Baksaas-Aasen ${ }^{1}$, Lewis Gall², Simon Eaglestone², Claire Rourke², Nicole. P. Juffermans ${ }^{3}$, J. Carel Goslings ${ }^{4}$, Paal Aksel Naess ${ }^{1}$, Susan van Dieren ${ }^{4}$, Sisse Rye Ostrowski ${ }^{8}$, Jakob Stensballe ${ }^{8}$, Marc Maegele ${ }^{5}$, Simon J. Stanworth ${ }^{6,7}$, Christine Gaarder $^{1 *}$, Karim Brohi ${ }^{2}$ and Per I. Johansson ${ }^{8}$

\begin{abstract}
Background: Traumatic injury is the fourth leading cause of death globally. Half of all trauma deaths are due to bleeding and most of these will occur within $6 \mathrm{~h}$ of injury. Haemorrhagic shock following injury has been shown to induce a clotting dysfunction within minutes, and this early trauma-induced coagulopathy (TIC) may exacerbate bleeding and is associated with higher mortality and morbidity. In spite of improved resuscitation strategies over the last decade, current transfusion therapy still fails to correct TIC during ongoing haemorrhage and evidence for the optimal management of bleeding trauma patients is lacking. Recent publications describe increasing the use of Viscoelastic Haemostatic Assays (VHAs) in trauma haemorrhage; however, there is insufficient evidence to support their superiority to conventional coagulation tests (CCTs).

Methods/design: This multicentre, randomised controlled study will compare the haemostatic effect of an evidence-based VHA-guided versus an optimised CCT-guided transfusion algorithm in haemorrhaging trauma patients. A total of 392 adult trauma patients will be enrolled at major trauma centres. Participants will be eligible if they present with clinical signs of haemorrhagic shock, activate the local massive haemorrhage protocol and initiate first blood transfusion. Enrolled patients will be block randomised per centre to either VHA-guided or CCT-guided transfusion therapy in addition to that therapy delivered as part of standard care, until haemostasis is achieved. Patients will be followed until discharge or 28 days. The primary endpoint is the proportion of subjects alive and free of massive transfusion (less than 10 units of red blood cells) at $24 \mathrm{~h}$. Secondary outcomes include the effect of CCT- versus VHA-guided therapy on organ failure, total hospital and intensive care lengths of stay, health care resources needed and mortality. Surviving patients will be asked to complete a quality of life questionnaire (EuroQol EQ-5D ${ }^{\mathrm{TM}}$ ) at day 90.
\end{abstract}

Discussion: CCTs have traditionally been used to detect TIC and monitor response to treatment in traumatic major haemorrhage. The use of VHAs is increasing, but limited evidence exists to support the superiority of these technologies (or comparatively) for patient-centred outcomes. This knowledge gap will be addressed by this trial.

Trial registration: ClinicalTrials.gov, ID: NCT02593877. Registered on 15 October 2015.

Trial sponsor

Queen Mary University of London

(Continued on next page)

\footnotetext{
* Correspondence: Tinagaar@online.no

'Department of Traumatology, Oslo University Hospital, Oslo, Norway

Full list of author information is available at the end of the article
} 
(Continued from previous page)

The contact person of the above sponsor organisation is: Dr. Sally Burtles, Director of Research Services and Business Development, Joint Research Management Office, QM Innovation Building, 5 Walden Street, London E1 2EF; phone: 0207882 7260; Email: sponsorsrep@bartshealth.nhs.uk

Trial sites

Academic Medical Centre, Amsterdam, The Netherlands

Kliniken der Stadt Köln gGmbH, Cologne, Germany

Rigshospitalet (Copenhagen University Hospital), Copenhagen, Denmark

John Radcliff Hospital, Oxford, United Kingdom

Oslo University Hospital, Oslo, Norway

The Royal London Hospital, London, United Kingdom

Centre for Trauma Sciences, Blizard Institute, Queen Mary University of London, London, United Kingdom Health Economics Research Centre, Nuffield Department of Population Health, University of Oxford, Oxford, United Kingdom

Sites that are planning to start recruitment in mid/late 2017

Nottingham University Hospitals, Queen's Medical Centre, Nottingham, United Kingdom

University of Kansas Hospital (UKH), Kansas City, MO, USA

Protocol version: 3.0/14.03.2017 (Additional file 1)

Keywords: Trauma, Haemorrhage, Trauma-induced coagulopathy, Viscoelastic haemostatic assays, Randomised control trial, Conventional coagulation tests, Transfusion

\section{Background}

Traumatic injury is responsible for a large and increasing proportion of the world's burden of disease and is the fourth leading cause of death globally [1]. Half of all trauma deaths are due to bleeding and most of these will occur within $6 \mathrm{~h}$ from injury [2]. Haemorrhagic shock following injury has been shown to induce a clotting dysfunction (i.e. coagulopathy) within minutes [3-5]. Such early traumainduced coagulopathy (TIC) may exacerbate bleeding and is associated with higher mortality and morbidity $[4,6,7]$. Many more injured patients will go on to develop different types of coagulopathy at different times during the course of their treatment, either as a result of their body's ongoing response to trauma or as a consequence of their clinical care. Coagulopathic, haemorrhaging trauma patients have increased blood transfusion requirements, increased mortality and more adverse outcomes [8]. Despite improvements in surgical techniques, resuscitation strategies and intensive care treatments, outcomes for critically injured patients remain poor [9]. Within the last decade research focussing on TIC has led to improved resuscitation strategies, resulting in the early and more aggressive use of blood products and coagulation factors for the management of massively bleeding patients.

In spite of improved resuscitation strategies, current transfusion therapy still fails to correct coagulopathy during ongoing haemorrhage $[10,11]$. The mechanisms and genesis of TIC have yet to be fully elucidated, and there are many questions about how to optimally diagnose, resuscitate and monitor the critically bleeding trauma patient. It is important to detect TIC as early as possible. Conventional coagulation tests (CCT), such as prothrombin time/international normalised ratio (PT/ INR), activated partial thromboplastin time (APTT), fibrinogen concentration and PLT, have traditionally been used. However, there is a striking lack of evidence to support the use of these CCTs to monitor resuscitation, although threshold triggers for intervention based on CCTs have been suggested [5]. Recent published evidence describes an increasing recognition for the potential of the two current market-leading Viscoelastic Haemostatic Assays (VHAs) namely thromboelastography $\left(\mathrm{TEG}^{\circ}\right.$; Haemonetics Incorporation) and rotational thromboelastometry (ROTEM ${ }^{\circ}$; TEM Innovation $\mathrm{GmbH}$ ). Both platforms use similar test modes to rapidly and accurately determine the functional coagulation status of patient whole blood. However, the evidence base supporting a role for these VHA devices is limited, and less attention has been directed to understanding their cost-effectiveness. Cost-effectiveness may be particularly relevant both in the context of additional therapeutic interventions required, but also in potential savings, if fewer treatments are required based on delivery of individualised assessments of haemostasis.

The relative contribution of blood components, such as fibrinogen and platelets, to clot strength can be evaluated through the use of specific inhibitors or agonists [12]. The viscoelastic properties of blood samples are recorded under low shear conditions, thereby providing a comprehensive visual profile of clot formation and breakdown (fibrinolysis). 
Unlike laboratory-based CCTs which might take more than 60 min for the results to be available to clinicians [8], VHA is a point-of-care device which might provide clinically relevant results within even 5-10 min and thus may be repeated in a massive bleeding situation to identify patient-specific needs for transfusion components in a timelier manner. Furthermore, VHAs provide the potential to detect hyperfibrinolysis, and possibly hypercoagulability. However, VHA assays and testing have costs, require training and additional oversight, and may not provide insight into other potentially important haemostatic derangements at the endothelial or platelet level. In addition, other publications attest to how changes in the process and pathways for the delivery of CCTs can be modified and accelerated [13, 14].

Whilst VHA has been used for many years in liver transplant and cardio-pulmonary surgery, robust data supporting its universal uptake in the context of trauma are lacking. Whilst some publications have attempted to identify VHA patterns and thresholds characterizing TIC and the need for massive transfusion in trauma patients, definitive evidence proving its superiority over CCTs in the diagnosis and management of coagulopathy in the acute setting is not available [15-18].

Although considered a preventable major cause of death, the management of coagulopathic bleeding in trauma patients remains primarily based upon retrospective registry studies of survival and extrapolating the results of transfusion practice performed in the elective, non-acute surgical setting. Treatment is diverse comprising the empiric transfusion of red blood cells (RBC) and clotting product supplements to patients, blind to the type and severity of TIC they may have - or indeed even if they do not have coagulopathy. It is well established that blood transfusion carries significant health risks both related to transmission of pathogens and to the development of transfusion reactions. Published in 2015, the results of the Pragmatic, Randomised Optimal Platelet and Plasma Ratios (PROPPR) trial [19] provide the best evidence to date for optimal trauma haemorrhage resuscitation. PROPPR demonstrated that an empiric massive transfusion protocol (MTP) aiming at a ratio of 1:1:1 of blood components ( $R B C$ 1: plasma 1 : platelets 1) administered from the early phase of care and during ongoing haemorrhage was associated with fewer exsanguinations in the initial $24 \mathrm{~h}(p=0.03)$ and a tendency towards improved 24-h survival $(p=0.12)$ than a $1: 1: 2$ ratio.

The present prospective randomised controlled trial (RCT) will employ evidence-based treatment algorithms to compare outcomes of VHA-guided resuscitation versus CCT resuscitation support in haemorrhaging trauma patients.

The hypothesis for this comparative study is that VHA-directed therapy will enhance early haemostatic control by the targeted correction of TIC, whilst also reducing the total amount of blood products and procoagulants administered to all bleeding trauma patients, including those not having TIC. This would significantly reduce both the number of patients receiving blood transfusion and the number of transfused blood products per transfused patient, thereby improving both patient safety and resource utilisation.

\section{Methods/design}

This is an investigator-initiated, multi-centred, superiority, parallel-group, randomised controlled trial performed at eight major trauma centres. The trial sites include: Rigshospitalet (Copenhagen, Denmark), Academic Medical Centre (Amsterdam, The Netherlands), Oslo University Hospital (Oslo, Norway), Kliniken der Stadt Köln gGmbH Cologne, Germany), The Royal London Hospital (London, UK), John Radcliffe Hospital (Oxford, UK). Nottingham University Hospitals, Queen's Medical Centre (Nottingham, United Kingdom) and University of Kansas Hospital (UKH) (Kansas City, MO, USA) are planning to start recruitment in 2017.

This protocol (Additional file 1) conforms to the Consolidated Standard of Reporting Trials (CONSORT) guidelines. Figure 1 shows the Standard Protocol Items: Recommendation for Interventional Trials (SPIRIT) schedule of enrolment, interventions and assessments. The SPIRIT Checklist is given in the Additional file 2.

An overview of the study process is provided in Fig. 2 (study scheme).

\section{Inclusion criteria}

Adult trauma patients (according to local definitions) will be enrolled if they present with clinical signs of haemorrhagic shock, according to the responsible trauma team leader, activate the local massive haemorrhage protocol, according to the participating institutions' specific routines, and initiate first transfusion. Participants must be randomised within $3 \mathrm{~h}$ of injury and $1 \mathrm{~h}$ of admission to the ED of the participating study site. Agreement is provided on behalf of incapacitated patients by a personal consultee (PC) or a nominated consultee (NC).

\section{Exclusion criteria}

There are no exclusion criteria.

\section{Primary objective}

The primary objective is to compare the haemostatic effect of VHA assay-guided transfusion strategy versus optimised CCT-guided transfusion strategy in haemorrhaging trauma patients. 


\begin{tabular}{|c|c|c|c|c|c|c|c|c|c|}
\hline & \multicolumn{9}{|c|}{ STUDY PERIOD } \\
\hline & \multirow{2}{*}{$\begin{array}{c}\text { Enrolment } \\
\begin{array}{c}\text { admittance } \\
\text { ED }\end{array} \\
\end{array}$} & \multirow{2}{*}{$\begin{array}{c}\text { Allocation } \\
0\end{array}$} & \multicolumn{7}{|c|}{ Post-allocation } \\
\hline TIME POINT & & & $\begin{array}{c}\text { Every } \\
4 U \\
R B C \\
\end{array}$ & $\begin{array}{c}\text { Haemo } \\
\text { stasis }\end{array}$ & $6 \mathrm{hrs}$ & $\begin{array}{c}24 \\
h r s\end{array}$ & $\begin{array}{c}\text { Until } \\
\text { day } 28\end{array}$ & $\begin{array}{c}\text { Dis- } \\
\text { charge }\end{array}$ & Day 90 \\
\hline \multirow{4}{*}{$\begin{array}{r}\text { ENROLMENT: } \\
\text { Eligibility screening } \\
\text { and randomisation } \\
\text { Informed consent } \\
\text { Allocation }\end{array}$} & & & & & & & & & \\
\hline & $\mathrm{X}$ & & & & & & & & \\
\hline & & $\mathrm{X}$ & & & & & & & \\
\hline & & $\mathrm{X}$ & & & & & & & \\
\hline \multicolumn{10}{|l|}{ INTERVENTIONS: } \\
\hline \multicolumn{10}{|l|}{$C C T$} \\
\hline \multicolumn{10}{|l|}{$V H A$} \\
\hline \multicolumn{10}{|l|}{ ASSESSMENTS: } \\
\hline \multicolumn{10}{|l|}{ Blood Sample } \\
\hline \multicolumn{10}{|l|}{ Arterial Blood Tests } \\
\hline Blood Chemistry & & $\mathrm{X}$ & & & & $\mathrm{X}$ & & & \\
\hline Haematology & & $\mathrm{X}$ & & & & $\mathrm{X}$ & & & \\
\hline \multicolumn{10}{|l|}{ Physical exam } \\
\hline \multicolumn{10}{|l|}{$\begin{array}{r}\text { Total blood, } \\
\text { Coagulation Factor, } \\
\text { Fluid and TXA }\end{array}$} \\
\hline SOFA & & $\mathrm{X}$ & & & & $\mathrm{X}$ & $\begin{array}{l}\text { end } \\
\text { ICU }\end{array}$ & $\mathrm{X}$ & \\
\hline SAEs & & $\vdash$ & & & & & & $\rightarrow$ & \\
\hline Mortality & & & & & $\mathrm{X}$ & $\mathrm{X}$ & $\mathrm{x}$ & & $\mathrm{x}$ \\
\hline Ventilator-free days & & & & & & & $\mathrm{X}$ & $\mathrm{X}$ & \\
\hline Vasopressor days & & & & & & & $\mathrm{X}$ & $\mathrm{X}$ & \\
\hline$R R T$ days & & & & & & & $\mathrm{X}$ & $\mathrm{X}$ & \\
\hline ICU days & & & & & & & $\mathrm{x}$ & $\mathrm{x}$ & \\
\hline Hospital days & & & & & & & $\mathrm{x}$ & $\mathrm{x}$ & \\
\hline Surgical episodes & & & & & & & $\mathrm{X}$ & $\mathrm{X}$ & \\
\hline Thromboembolic events & & & & & & & $\mathrm{X}$ & $\mathrm{X}$ & \\
\hline First destination & & & & & & & & $\mathrm{X}$ & \\
\hline Quality of Life & & & & & & & & $\mathrm{x}$ & $\mathrm{X}$ \\
\hline Current disposition & & & & & & & & & $\mathrm{X}$ \\
\hline
\end{tabular}

Fig. 1 Standard Protocol Items: Recommendation for Interventional Trials (SPIRIT) schedule of enrolment, interventions and assessments

\section{Secondary objective}

The secondary objectives of the study are to determine the effects of VHA-led versus optimised CCT-guided resuscitation on organ failure, hospital length of stay (LOS), intensive care unit (ICU) stay, duration of mechanical ventilation, health care resource needs and mortality.

\section{Primary endpoint}

The primary endpoint is the proportion of subjects alive and free of massive transfusion (less than 10 units of $\mathrm{RBC}$ transfused) at $24 \mathrm{~h}$ post admission.

\section{Secondary endpoints}

The secondary endpoints listed below will be analysed in order to provide a sensitive and comprehensive description of outcomes and health care resource demands for the VHA and CCT arm subjects:
- All-cause mortality at 6 and $24 \mathrm{~h}$ and 28 and 90 days post admission

- Duration and severity of coagulopathy until haemostasis, as defined by the area under the time multiplied by Prothrombin Ratio (PTr) curve, with coagulopathy defined as PTr $>1.2$. Patients who die will have their time of haemostasis set at $24 \mathrm{~h}$, and last PTr extrapolated to this time point

- Proportion of patients who have corrected coagulopathy, defined as $\operatorname{PTr}<1.2$, after first 8 units of RBC

- Time to haemostasis (defined as having occurred at the end of the first hour free of red cell transfusions and the treating clinicians believe primary haemostasis has been achieved)

- Time spent in coagulopathic condition, defined as $\mathrm{PTr} \geq 1.2$, until haemostasis, defined as the point $1 \mathrm{~h}$ 


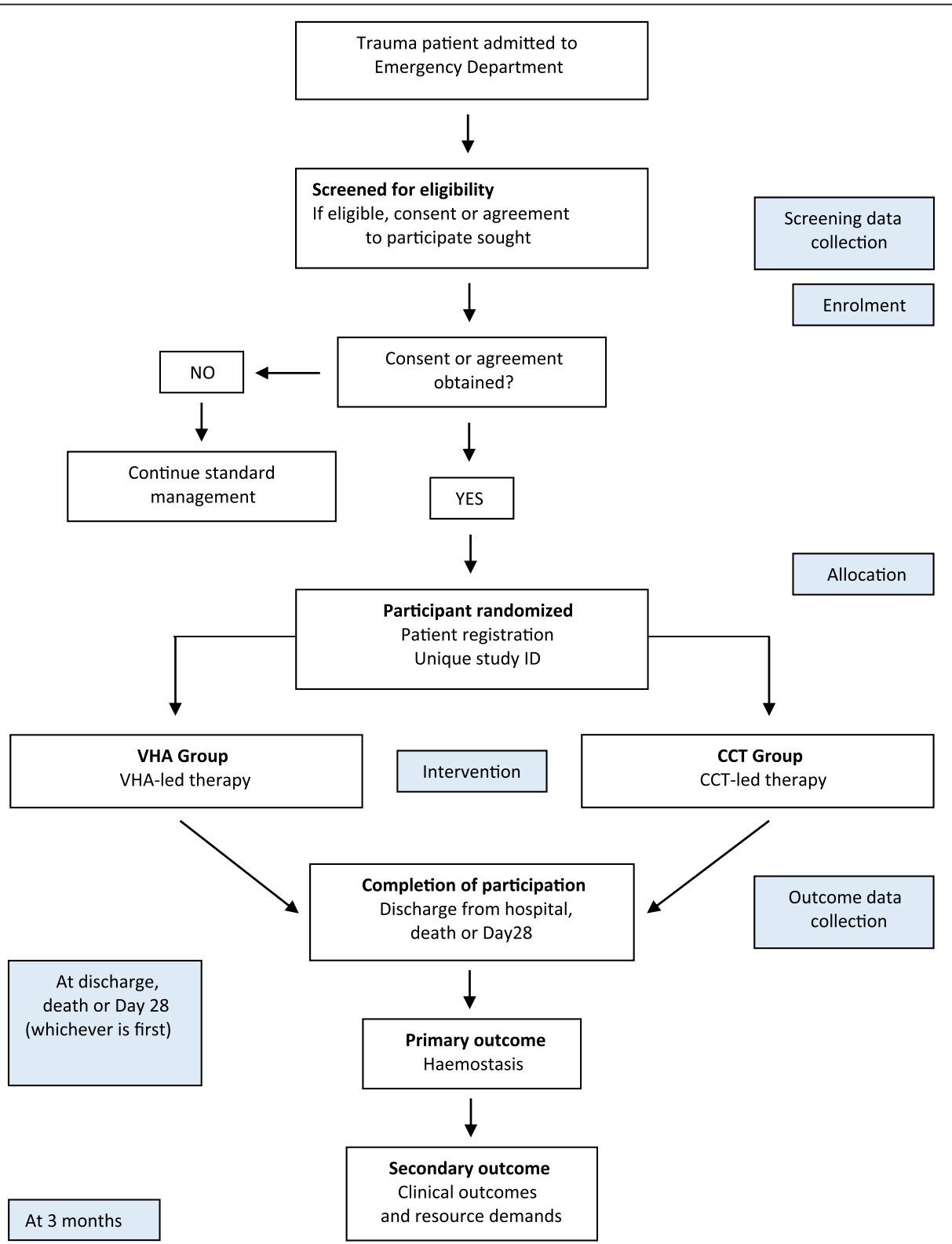

Fig. 2 Study scheme

from the last administration of $\mathrm{RBC}$ and the treating clinician believes that primary haemostasis has been achieved

- Blood products administered (RBC, plasma, platelets alone and in total) within first 6 and $24 \mathrm{~h}$ after admission

- 28-day ventilator-free and ICU-free days (patients who die in hospital during the 28-day study period will be considered to have zero hospital-free days)

- Total hospital length of stay

- 28-day symptomatic thromboembolic events defined as: deep venous thrombosis (DVT) diagnosed by compression ultrasound or venography, pulmonary embolism diagnosed by computed tomography (CT) pulmonary angiogram or ventilation-perfusion scan or myocardial infarction and/or stroke identified by standard clinical diagnostic investigation(s)

- Incidence of transfusion-related complications

- Incidence of organ dysfunction, defined by Sequential Organ Failure Assessment (SOFA) score

- Health care resource, productivity costs and HRQoL (EuroQol EQ-5DTM at discharge or day 28, and at day 90)

- Lifetime health economic cost-effectiveness of personalised VHA-guided haemorrhagic treatment versus MTP-based on best practice and CCTs 


\section{Study procedures}

\section{Screening procedures}

Local investigators will identify eligible adult trauma patients with haemorrhagic shock and ongoing bleeding as soon as possible after the patient has arrived in the emergency department (ED), using local transfusion triggers. If patients are deemed to be eligible, consent for entry into the trial will be sought. A screening log will be completed once a week, which will record all patients considered for eligibility to the trial by the investigator(s). The log will include age, gender, inclusion/exclusion criteria and other reasons for non-enrolment. The screening log data will be reviewed at regular intervals.

\section{Randomisation procedure}

Enrolled patients will be block randomised per centre to either the CCT or the VHA study arm within $3 \mathrm{~h}$ of injury and within $1 \mathrm{~h}$ of admission. The trial will be un-blinded for clinical staff and site investigators. Once a patient is determined eligible for the study and informed consent or agreement has been obtained, each subject will be enrolled as soon as possible and will be assigned a unique alphanumeric study identifier. Randomisation will be performed by the site investigator opening a sealed envelope containing the randomised treatment group, to allow for immediate allocation of subjects. An independent party, appointed by the sponsor, will generate the randomisation sequence and site envelopes centrally.

\section{Schedule of intervention}

All participating centres will initiate the management of the study population according to standard local protocols regardless of enrolment in the trial. Following randomisation, study participants will undergo interventions at set time points as outlined in Fig. 1 (Standard Protocol Items: Recommendations for Interventional Trials (SPIRIT) schedule of enrolment, interventions and assessments) and be followed up for 90 days after enrolment.

\section{Standard care}

All participating centres currently manage critically bleeding trauma patients according to a standardised MTP aiming at a ratio of $\mathrm{RBC} 1$ : plasma 1: platelets 1 (1:1:1), typically administering plasma from the start of resuscitation and platelets immediately as they become available. Tranexamic acid (TXA) will be administered to all patients as an intravenous bolus of $1 \mathrm{~g}$ over 10 min (either pre-hospital or in the ED) followed by an intravenous infusion of $1 \mathrm{~g}$ over $8 \mathrm{~h}$, providing that the patient is less than $3 \mathrm{~h}$ post injury.

Current use of additional diagnostics and therapy, such as systematic approach according to Advanced Trauma Life Support (ATLS ${ }^{\ominus}$ principles, early imaging (e.g. X-rays, Focussed Assessment with Sonography for Trauma (FAST), computer tomography $(\mathrm{CT})$ ), activation criteria for MTP, surgical approach applying damage control principles when indicated, the availability and use of interventional radiology, will not be affected in either of the study groups. An optimised initial MTP based on a 1:1:1 balanced transfusion will be implemented in all centres for approximately 2 months prior to initiation of the RCT and standardised as far as local routines and blood product availability allow.

\section{Initiation of study care}

Corresponding and optimised algorithms based on VHA trigger parameters for the VHA arm and CCT results for the CCT arm, respectively, have been developed and will be applied in the enrolled subjects (Fig. 3a, b, c). During active haemorrhage, samples will be taken for CCT/VHA analysis at baseline and after every 4 units of RBC until haemostasis. The results from each blood sample will be acted upon as soon as they are available. For the VHA arm, this implies acting upon the parameters as they are appearing, not waiting until the VHA trace is completed.

If a planned study intervention has not yet been administered, the sample will be taken and analysed (where resources allow) but will not be used to guide study intervention. The first sample taken after a study intervention is actually administered will be the next sample used to guide study intervention based upon the respective protocol.

The same blood products and procoagulants will be employed in both study arms, with existing standard practice in all participating centres being closely aligned to that of the CCT arm. Trial products will be given as an addition to the 1:1:1 baseline MTP and TXA. The procoagulants included in the algorithms are fibrinogen concentrate or cryoprecipitate and additional platelets, plasma and TXA.

Enrolled patients will be block randomised per centre to either study arm:

- CCT: haemostatic resuscitation (standard care), based on a MTP aiming at ratio 1:1:1 of blood components (RBC 1: plasma 1: platelets 1 ) and TXA, and CCTs to guide further resuscitation with blood products and procoagulant factors

- VHA: haemostatic resuscitation (standard care), based on a MTP aiming at ratio 1:1:1 of blood components (RBC 1: plasma 1: platelets 1 ) and TXA, and VHA-guiding further resuscitation with blood products and procoagulant factors

\section{Randomised study care - VHA arm}

VHA will be conducted for all subjects in the VHA arm at each time point up to $24 \mathrm{~h}$. During active 


\begin{tabular}{|c|c|c|c|}
\hline & a ROTEM & b TEG & C $\mathrm{CCT}$ \\
\hline & $\begin{array}{l}\text { FIBRINOGEN } \\
\text { If FIBTEM CA5 }<10 \mathrm{~mm} \\
\text { Give additional } 4 \mathrm{~g} \text { equivalent of } \\
\text { fibrinogen } \\
\text { (as cryoprecipitate or concentrate) }\end{array}$ & $\begin{array}{l}\text { FIBRINOGEN } \\
\text { If } \mathrm{FF} \text { TEG MA }<20 \mathrm{~mm} \\
\text { Give additional } 4 \mathrm{~g} \text { equivalent of } \\
\text { fibrinogen } \\
\text { (as cryoprecipitate or concentrate) }\end{array}$ & $\begin{array}{l}\text { FIBRINOGEN } \\
\text { If Fibrinogen }<2 \mathrm{~g} / \mathrm{L} \\
\text { Give additional } 4 \mathrm{~g} \text { equivalent of } \\
\text { fibrinogen } \\
\text { (as cryoprecipitate or concentrate) }\end{array}$ \\
\hline & $\begin{array}{l}\text { PLATELETS } \\
\text { If (EXTEM CA5 - FIBTEM CA5) < } 30 \\
\mathrm{~mm} \\
\text { Give } 1 \text { additional pool of platelets }\end{array}$ & $\begin{array}{l}\text { PLATELETS } \\
\text { If ( } r \text { TEG MA - FF TEG MA) < } 45 \mathrm{~mm} \\
\text { Give } 1 \text { additional pool of platelets }\end{array}$ & $\begin{array}{l}\text { PLATELETS } \\
\text { If platelets }<100 \times 10^{9} / \mathrm{L} \\
\text { Give } 1 \text { additional pool of platelets }\end{array}$ \\
\hline & $\begin{array}{l}\text { PLASMA } \\
\text { If EXTEM CA5 } \geq 40 \mathrm{~mm} \text { AND } \\
\text { EXTEM CT > } 80 \mathrm{~s} \\
\text { Give } 4 \text { additional units of plasma }\end{array}$ & $\begin{array}{l}\text { PLASMA } \\
\text { If rTEG MA } \geq 65 \mathrm{~mm} \text { AND } \\
\text { rTEG ACT > } 120 \mathrm{~s} \\
\text { Give } 4 \text { additional units of plasma }\end{array}$ & $\begin{array}{l}\text { PLASMA } \\
\text { If INR }>1.2 \text { AND Fibrinogen } \geq 2 \mathrm{~g} / \mathrm{L} \\
\text { Give } 4 \text { additional units of plasma }\end{array}$ \\
\hline & $\begin{array}{l} \\
\text { TRANEXAMIC ACID } \\
\text { If EXTEM LI30 }<85 \% \\
\text { Give additional } 1 \mathrm{~g} \text { tranexamic acid }\end{array}$ & $\begin{array}{l}\overline{\text { TRANEXAMIC ACID }} \\
\text { If rTEG LY30 > } 10 \% \\
\text { Give additional } 1 \mathrm{~g} \text { tranexamic acid }\end{array}$ & \\
\hline gorithms & & & \\
\hline
\end{tabular}

haemorrhage, samples will be taken for VHA analysis at baseline and after every 4 units of $\mathrm{RBC}$ until haemostasis. The clinical course of subjects randomised to the VHA arm will follow a treatment algorithm utilizing VHA results (Fig. $3 \mathrm{a}$ and b). The subject will be treated with standard haemostatic resuscitation based on a MTP aiming for 1:1:1 ratio of blood components and initial TXA. In addition, according to threshold values in the algorithms, the subjects will be given fibrinogen concentrate or cryoprecipitate and/or additional platelets, plasma and TXA depending on the VHA results.

Fibrinogen concentrate or cryoprecipitate will be administrated when FIBTEM Clot Amplitude at 5 min (CA5) is below $10 \mathrm{~mm}$ or functional fibrinogen (FF) $\mathrm{TEG}^{\odot}$ Maximum Amplitude (MA) is below $20 \mathrm{~mm}$. Additional platelets are indicated when the subtracted amplitude of FIBTEM CA5 from EXTEM CA5 is below $30 \mathrm{~mm}$, or the subtracted amplitude of $\mathrm{FF} \mathrm{TEG}^{\bullet} \mathrm{MA}$ from rapid TEG $^{\circ} \mathrm{MA}$ is below $45 \mathrm{~mm}$. Additional plasma is indicated when the results show a normal EXTEM CA5, defined as above $40 \mathrm{~mm}$, but still a prolonged EXTEM clotting time (CT), defined as above $80 \mathrm{~s}$, or normal rapid $\mathrm{TEG}^{\odot} \mathrm{MA}$, defined as above $65 \mathrm{~mm}$, but still a prolonged rapid $\mathrm{TEG}^{\odot}$ activated clotting time (ACT), defined as above 120 s. Additional TXA is indicated when the EXTEM Lysis Index at 30 min (LI30) is below $85 \%$ or rapid TEG $^{\circledR}$ clot lysis at $30 \mathrm{~min}$ (Ly30) is above $10 \%$.
This VHA treatment algorithm is based upon analysis of more than 2200 trauma subjects enrolled to a prospective observational study conducted at the participating study sites, entitled Activation of Coagulation and Inflammation in Trauma (ACIT). Analysis of the ACIT dataset has enabled the definition of clinically relevant VHA thresholds and patterns by which it is possible to rapidly identify coagulopathic patients and anticipate the need for massive transfusion. These threshold parameters have been applied to the generation of an evidencebased targeted treatment algorithm.

According to pre-designation, each study centre will only conduct VHA using either thromboelastography $\left(\mathrm{TEG}^{\oplus}\right)$ or rotational thromboelastometry $\left(\mathrm{ROTEM}^{\oplus}\right)$ to determine the following parameters:

- $\mathrm{TEG}^{\oplus}$ : RapidTEG ${ }^{\oplus} \mathrm{ACT}, \mathrm{MA}$ and Ly30; functional fibrinogen $\mathrm{TEG}^{\oplus} \mathrm{MA}$

- ROTEM ${ }^{\bullet}$ : EXTEM CT, CA5 and Li30; FIBTEM CA5

\section{Randomised study care - CCT arm}

CCTs will be conducted for all subjects in the CCT arm at each time point up to $24 \mathrm{~h}$. The tests will comprise platelet counts (PLT), activated partial thromboplastin time (aPTT), prothrombin time - international normalised ratio (PT/INR) and Clauss fibrinogen assay. PTr and Clauss fibrinogen will be measured for all study subjects at each time point. 
The clinical course of subjects randomised to the CCT arm will follow a treatment algorithm utilizing CCT results (Fig. 3c) and based upon current published evidence and empiric best practice according to the PROPPR and CRASH-2 trials data (i.e. a 1:1:1 product ratio, with the anti-fibrinolytic TXA) [19-21]. The subject will be treated with standard haemostatic resuscitation based on a MTP aiming for a 1:1:1 ratio of blood components and initial TXA. In addition, the subjects will be given fibrinogen concentrate or cryoprecipitate and/or additional platelets and plasma depending on the CCT results.

Fibrinogen concentrate or cryoprecipitate will be indicated when fibrinogen values are below $2.0 \mathrm{~g} / \mathrm{L}$. Additional platelets will be indicated with PLT below $100 \times 10^{9} / \mathrm{L}$ and additional plasma will be administrated when the INR is above 1.2 despite normal fibrinogen, defined as $2.0 \mathrm{~g} / \mathrm{L}$ or above. There are no generally accepted indications for additional antifibrinolytic therapy using CCTs.

\section{Detail of outcome measures collected}

SOFA score (Additional file 3)

SOFA score will be registered until discharge from ICU.

\section{Blood products and procoagulants}

Timings, total number (and doses if appropriate) of different blood products and procoagulants administered both pre-hospital and after admission to the study centre, during resuscitation and after 6 and $24 \mathrm{~h}$ will be recorded including:

- $\mathrm{RBC}$, fresh frozen plasma (FFP)/Octaplas, cryoprecipitate, platelets, whole blood and/or autologous RBC from cell salvage

- Coagulation factor concentrates (prothrombin complex concentrate (PCC), fibrinogen, activated recombinant factor VII (rFVIIa))

- TXA

\section{Fluids}

Timings (during first $24 \mathrm{~h}$ only) and total volume of different fluids administered both pre-hospital and after admission to the study centre until $24 \mathrm{~h}$ will be recorded including crystalloids, colloids and hypertonic saline.

\section{Thromboprophylaxis/prothrombotic medication}

Type of medication administered, timings, dose and indication will be recorded daily until day 28 with particular attention to duration of treatment (stop date).

\section{Bleeding episodes}

Qualifying episodes will be defined by radiological evidence, like contrast extravasation on CT scan, and/or clinical suspicion, like haemodynamic instability, combined with transfusion requirement after initial haemostasis (defined as the point $1 \mathrm{~h}$ from the last administration of RBC and the treating clinician believes primary haemostasis has been achieved).

\section{Ventilator-free days}

Calculated by the subtracting the number of days spent on mechanical ventilation from 28. Death before day 28, recorded as 28 .

\section{Vasopressor days}

Calculated as the total number of days spent on inotropic drugs, including for instance noradrenaline, dobutamine, vasopressin.

\section{Renal replacement therapy days}

Calculated as the number of days spent on haemodialysis or haemofiltration.

\section{ICU days}

The total length of stay in the ICU. If the patient is in the ICU at any time point during a calendar day, this day will be considered an ICU day.

\section{Length of stay}

Length of stay will be recorded in days, for the total number spent in ICU and in hospital. If the patient is in the hospital at any time point during a day, this day will be considered a hospital day.

\section{Surgical episodes}

Description, timing, duration and reasons for all surgical episodes will be recorded. This includes interventional radiology and bedside surgical interventions in addition to major surgical procedures.

\section{Thromboembolic events}

Symptomatic venous thromboembolic events will be recorded, as confirmed by either compression ultrasound/ venography (DVT or by CT - pulmonary angiogram/ ventilation - perfusion scan (pulmonary embolism (PE)). Other thromboembolic events, such as myocardial infarction and/or stroke, will be identified by standard clinical diagnostic investigations(s).

\section{Patient disposition}

First destination after discharge and disposition at 90 days post admission will be recorded as either home, rehabilitation facility, nursing home, other hospital or other. 


\section{Quality of life (Qol)}

Subject quality of life will be assessed using the EuroQoL EQ-5D ${ }^{\mathrm{ma}}$ questionnaire, a standardised instrument for use as a measure of health outcome. Quality of life assessment will be conducted in the study centre upon discharge of the subject from hospital and at 90 days post admission.

The in-hospital (i.e. discharge) questionnaire will be conducted by research investigators with the patient where possible, but may also be completed with patient's PC if necessary. The questionnaire can be completed in less than $5 \mathrm{~min}$. Where the subject has already left hospital, the questionnaire will be posted out with a return stamped addressed envelope.

Patients who have not returned the questionnaire within 2 weeks of the initial request will be telephoned as a reminder to complete the questionnaire and may be asked to complete it over the telephone if necessary.

A further EuroQoL EQ-5D ${ }^{\mathrm{TM}}$ questionnaire will be provided to assess subject quality of life at $90 \pm 5$ days post admission. Confirmation with the local (i.e. hospital care record system) and regional resources (i.e. NHS Health and Social Care Information Centre Spine Services) will ensure that only surviving patients receive a questionnaire.

\section{Cessation of study care (haemostasis)}

For the purposes of this comparative study, haemostasis (end of study care) will be defined as the point $1 \mathrm{~h}$ from the last administration of RBC and the treating clinician believes that primary haemostasis has been achieved.

\section{Procedure for data collection}

Study subject data will be captured locally using a paper Case Report Form (CRF), following local data security routines. CRF data are transferred and uploaded to a centralised study database whereupon study data integrity is reviewed weekly by the Trial Coordinating Centre.

\section{Adverse event reporting}

Patients included in this trial have a high risk of morbidity and mortality, with either treatment being administered during a phase of critical bleeding and circulatory failure. Therefore, patients have a very high risk of experiencing several adverse events (AEs) and serious adverse events (SAEs). All SAEs, expected or not, will be recorded on a SAE form. Any SAE, death or thromboembolic or ischaemic events (myocardial infarction, stroke, pulmonary embolus, DVT) that are considered to be 'related' and unexpected are to be reported to the sponsor within $24 \mathrm{~h}$, and to the Main Research Ethics Committee (MREC) within 15 days in line with the required timeframe.

\section{Urgent safety measures}

The chief investigator (CI) will take urgent safety measures to ensure the safety and protection of the clinical trial subjects from any immediate hazard to their health and safety. The measures should be taken immediately. In this instance, the approval of the Ethics Committee prior to implementing these safety measures is not required. However, it is the responsibility of the CI to inform the sponsor and the Main Research Ethics Committee (MREC) (via telephone) of this event immediately.

The CI has an obligation to inform both the MREC in writing within 3 days, in the form of a substantial amendment. The sponsor (Joint Research Management Office (JRMO)) must be sent a copy of the correspondence with regards to this matter.

\section{Annual safety reporting}

The CI will send the Annual Progress Report to the MREC using the NRES template (the anniversary date is the date on the MREC 'favourable opinion' letter from the MREC) and to the sponsor.

\section{Subject withdrawal}

Every reasonable effort will be made to maintain protocol compliance and to retain patient participation in the study, consistent with the provisions of informed consent and good clinical practice. The following are potential reasons why a patient may be withdrawn from the study:

1. Withdrawal of consent/agreement

2. Retrospective exclusion: if a patient is deemed to not meet one or more of the inclusion/exclusion criteria in retrospect they will be withdrawn from the study

3. Major protocol deviation from the study design by the subject, observed or suspected by the investigator

4. Administrative: the sponsor or monitoring committees decide to terminate or discontinue the study

5. The subject's health would be jeopardised by continued participation and hence will be withdrawn at the discretion of the investigator

The study withdrawal form will be completed for these patients and a reason for withdrawal captured. All subject's withdrawn from the study will be managed in accordance with the hospital's standard procedures.

\section{Data collection and follow-up for withdrawn subjects}

Patients who withdraw from the study after randomisation will be followed for safety by conducting safety assessments through to the end of day 28. If a patient who 
withdraws has an ongoing SAE every effort must be made to follow up such events until satisfactory resolution is obtained or until further follow-up is no longer warranted.

\section{Subject replacement}

Subjects who withdraw from the study will be replaced.

\section{End of study definition}

The study will be considered closed when all surviving subjects complete in-hospital safety and outcome monitoring. This includes: safety measures of SAE rate within 28 days, total hospital stay, total critical care stays, 28day ventilator-free days and 28-day mortality

\section{Statistical considerations Sample size}

Based upon legacy registry data from the partners, approximately $28 \%$ of patients will need massive transfusion or die. It is expected that this figure will decrease to an overall proportion of $15 \%$ in the VHA group (i.e. using VHA-guided strategy). In order to detect a difference from $28 \%$ to $15 \%$ with a power of $80 \%$ and a twosided alpha of $0.05,170$ patients per group are required. One hundred and ninety-six patients per study arm allows a $13 \%$ dropout rate, with an allocation ratio of 1 . The planned sample size for this study is 392 patients for which MTP is activated and transfusions initiated, 196 in each study arm.

\section{Method of analysis}

All primary and secondary outcomes will be analysed as intention-to-treat, and will include all randomised patients for whom the primary outcome of 'alive' and 'free of massive transfusion' at $24 \mathrm{~h}$ is recorded. The primary endpoint of patients who are alive and free of massive transfusion at $24 \mathrm{~h}$ will be assessed by difference in proportion with $95 \%$ confidence intervals. The chi-square test or Fischer's exact test will be used were appropriate. Absolute risk reduction and relative risk reduction by VHA-guided therapy will be calculated.

Kaplan-Meier mortality estimates between the two arms for all-cause mortality at 6 and $24 \mathrm{~h}$, as well as 28 and 90 days post admission, will be estimated for the secondary endpoint of death.

A per-protocol analysis and a sensitivity analysis will be performed for the primary endpoint. The following patients will be excluded from the per-protocol analysis:

- Patients who do not have at least one ROTEM $\%$ TEG $/$ CCT test performed and

- Who die within 60 min after baseline blood sampling or
- Who achieve haemostasis within $60 \mathrm{~min}$ of baseline sampling

Both ROTEM $^{\bullet}$-guided and $\mathrm{TEG}^{\circ}$-guided therapy together (i.e. the VHA arm) will be compared with the CCT arm. Separate analyses will be performed for ROTEM $^{\bullet}$-guided and TEG ${ }^{\circ}$-guided therapy alone for primary endpoints and correction of coagulopathy.

All applied tests will be two-sided and $p$ values of 0.05 will be accepted as statistically significant. We will report $p$ values with and without correction for multiple testing.

\section{Subgroup analyses}

The following patient categories will be included in all primary and secondary analyses but will also be analysed separately as subgroup analyses:

- Patients with known pre-existing coagulopathy

- Oral anticoagulant therapy (except for aspirin)

- Excluding patients with severe traumatic brain injury (AIS brain 4,5 or 6)

- Patients who arriving in a coagulopathic state (PTr $>1.2$ )

- Patients who received a massive transfusion (10 or more RBC units in the first $24 \mathrm{~h}$ )

\section{Sensitivity analyses}

Missing data are not expected for the primary outcome. Sensitivity analysis for secondary outcomes will be assessed using 100 multiple imputations for missing data. Rubin rules will be used to summarise the results of the multiple imputations.

\section{Integrated cost-effectiveness analysis}

A cost-effectiveness analysis will be conducted to assess the costs and effects of VHA-guided therapy versus those of optimised empiric treatment. A model will be developed which will be structured around the key clinical time points and events in the early management pathway of bleeding trauma patients.

The two treatment policies will be compared in terms of their estimated costs and effects (quality-adjusted life years (QALYs): calculated by combining survival and HRQoL data) and incremental analyses will be performed. If VHA-guided therapy is more effective but also more costly than empirical treatment, then the incremental cost-effectiveness ratio (ICER) will be calculated. The ICER is calculated by dividing the difference in costs between VHA and empirically guided therapy by the difference in effects (QALYs) and gives the additional cost of generating one additional unit of outcome (here, a QALY). 
So as to account for the uncertainty in the model input data, parameters will be entered as distributions rather than point estimates. Probabilistic sensitivity analysis will be used to take repeated random draws from all distributions simultaneously, each time recalculating the model's results for a total of 2000 times. The uncertainty will be summarised on the cost-effectiveness plane and using cost-effectiveness acceptability curves. For each country, the modelling exercise should provide an estimate of the probability that VHA-guided therapy is likely to be cost-effective when compared with optimised empiric treatment.

\section{Monitoring and quality assurance Summary monitoring plan}

Data coordination and site management services will be performed at the sponsor institution, Queen Mary University of London. The site clinical trials coordinator will perform regular monitoring of trial documentation and CRFs.

\section{Safety analysis}

A pre-defined interim analysis will be performed after the enrolment of 100 patients, including an assessment of recruitment logistics with the possibility to revise the planned sample size.

A Data Safety Monitoring Board (DSMB) will review all data on outcome of the patients in the respective treatment arms. The DSMB will focus on adherence to protocol, and present pre-specified criteria that need to be fulfilled with regard to patient safety for the study to continue.

\section{Audit and inspection}

For the purpose of compliance with Good Clinical Practice (GCP) and Regulatory Agency Guidelines it may be necessary for the sponsor or a drug regulatory agency to conduct a site audit. This may occur at any time from the start to after conclusion of the study.

\section{Discussion}

TIC is present early after injury in a significant proportion of patients [3-5], and is associated with increased bleeding, greater risk of complications and increased mortality, underlining the importance of early detection and aggressive treatment $[4,6,7,22]$.

Improvements in transfusion strategies over the last decade are associated with better outcome [23-26]. The results of the PROPPR trial [19] provide the best evidence to date for ratio-based trauma resuscitation. In that study, a MTP aiming at a 1:1:1 ratio of plasma 1: platelets 1: RBC 1 until haemorrhage control was associated with better outcome than a 1:1:2 ratio. However, PROPPR did not allow adjustments in the individual treatment based on results from coagulation tests during the course of resuscitation.

Traditionally, CCTs such as prothrombin time/international normalised ratio (PT/INR), activated partial thromboplastin time (aPTT), fibrinogen concentration and PLT have been recommended to guide resuscitation in bleeding trauma patients [27]. However, none of the existing CCTs have proven to be robust in detecting TIC or predicting massive transfusion. Moreover, CCTs are time-consuming laboratory tests only reflecting the initial steps of blood coagulation and not taking into account the interaction between platelets and coagulation factors.

On that background, the potential benefit of Viscoelastic Haemostatic Assays (VHAs), such as $\mathrm{TEG}^{\circ}$ and ROTEM $^{\oplus}$, in the trauma setting has gained much attention over the last decade. VHAs are dynamic tests; they may be performed bedside with their first results available within minutes of initiation. Several algorithms for guiding resuscitation in bleeding trauma patients based on VHA parameters have been published [28, 29]. None of them have been developed based on real-time large cohorts of trauma patients.

The updated European guidelines addressing the management of bleeding and coagulopathy following major trauma recommend the use of viscoelastic methods to assist in characterising the coagulopathy and in guiding haemostatic therapy [27] although the evidence to support the use of VHAs in this category of patients is insufficient [15-18].

Based on limited existing evidence to support the use of VHA versus CCTs in monitoring the resuscitation of massively bleeding trauma patients, our aim is to evaluate the differences between VHA-guided and CCTguided transfusion in trauma patients, and to create robust evidence-based guidelines for massive transfusion in trauma patients.

iTACTIC has obvious limitations, based on the actual level of evidence in this field. The challenges include the heterogeneity of, and access to, a population of severely injured patients as well as the development of relevant algorithms. Strength and weaknesses will be fully addressed when the trial results are published.

\section{Trial status}

This study is ongoing and started recruiting June 2016. Recruitment will be completed mid 2018.

\section{Additional files}

Additional file 1: Protocol version 3.0/14.03.2017. (PDF $2172 \mathrm{~kb}$ )

Additional file 2: SPIRIT Checklist. (DOC $129 \mathrm{~kb}$ )

Additional file 3: SOFA score. (ZIP $217 \mathrm{~kb}$ ) 


\section{Abbreviations}

ACIT: Activation of Coagulation and Inflammation in Trauma; ACT $\left(\right.$ ROTEM $\left.^{\circledast}\right)$ : Activated clotting time $\left(\right.$ ROTEM $\left.^{\circledR}\right)$; AE: Adverse event; APTT: Activated partial thromboplastin time; ATLS ${ }^{\oplus}$ : Advanced Trauma Life Support; CA $5\left(\right.$ ROTEM $^{\oplus)}$ : Clot Amplitude at 5 min (ROTEM $\left.{ }^{\oplus}\right)$; CCT: Conventional coagulation tests; Cl: Chief investigator; CONSORT: Consolidated Standards of Reporting Trials; CRF: Case Report Form; CT: Computer tomography; CT (ROTEM ${ }^{\oplus}$ ): Clotting time $\left(\right.$ ROTEM $\left.^{\oplus}\right)$; DSMB: Data Safety Monitoring Board; DVT: Deep venous thrombosis; ED: Emergency department; FAST: Focussed Assessment with Sonography in Trauma; FF (TEG): Functional fibrinogen (TEG $\left.{ }^{\circledast}\right)$; FFP: Fresh frozen plasma; GCP: Good Clinical Practice; ICER: Incremental cost-effectiveness ratio; ICU: Intensive care unit; JRMO: Joint Research Management Office; LI30 $\left(\right.$ ROTEM $^{\oplus)}$ : Lysis Index at 30 min $\left(\right.$ ROTEM $^{\oplus)}$; $\left(\right.$ TEG $\left.^{\oplus}\right)$ : Clot lysis at 30 min (TEG ${ }^{\circledast}$; MA: Maximum Amplitude; MREC: Main Research Ethics Committee; MTP: Massive transfusion protocol; PC: Personal consultee; PCC: Prothrombin complex concentrate; PE: Pulmonary embolism; PROPPR: Pragmatic, Randomised Optimal Platelet and Plasma Ratios trial; PTr: Prothrombin Time/ International Ratio (PT/INR); QALY: Quality-adjusted Life Years; QMUL: Queen Mary University of London; QoL: Quality of life; RBC: Red blood cells; RCT: Randomised controlled trial; rFVIla: Activated recombinant factor VII: ROTEM $^{\oplus}$ : Rotational thromboelastometry; SAE: Serious adverse event; SOFA: Sequential Organ Failure Assessment; SPIRIT: Standard Protocol Items: Recommendations for Interventional Trials; Subject: An individual who takes part in a clinical trial; TEG ${ }^{\oplus}$ : Thromboelastography; TIC: Trauma-induced coagulopathy; TXA: Tranexamic acid; VHA: Viscoelastic Haemostatic Assays

\section{Acknowledgements}

R Bergman, H Campbell, N Curry, A Aslani, S Beer, A Espinosa, EK Stürmer, N Schäfer, A Driessen, A Orr, A Schubert, KM Kolstadbraaten, N Flåten, K Balvers, M Wirtz, MW Hollmann.

\section{Funding}

This trial is part-funded by the European Commission under the FP-7 HEALTH-Contract No. F3-2013-602771, entitled 'Targeted Action for Curing Trauma Induced Coagulopathy' (TACTIC) [30]

Both $\mathrm{TEM}^{\circledast}$ International $\mathrm{GmbH}$ and Haemonetics ${ }^{\oplus}$ are equal partners in the TACTIC programme, providing VHA devices and reagents for all participating institutions.

\section{Availability of data and materials}

Data collection for this study will be accomplished using a paper CRF to capture data prospectively and transferred to an electronic data capture system (Discovere). The dataset will not be open access, but available upon request under the terms of Collaboration Agreement with Consortium.

\section{Authors' contributions}

PIJ, KB, MM, CG, SE, JCG, SJS and SE participated in the design of the study and achieved funding. PIJ, KB, CG, SJS, MM, JS, SRO, SVD, PAN, JCG, NPJ, CR, $\mathrm{SE}, \mathrm{LG}$ and KBA all contributed to protocol development. LG, KBA, CG, KB and PIJ prepared the first draft of the manuscript. All authors read and approved the final manuscript.

\section{Authors' information}

Not applicable

\section{Ethics approval and consent to participate}

This study has been approved by the respective Ethics Committees of the investigators' centers: Queen Mary University of London and John Radcliff Hospital, UK, reference number 16/LO/0004; Oslo University Hospital, Norway, reference number 2015/1601/REK sør-øst; Rigshospitalet, Denmark, reference number H-15017330; Academic Medical Centre, The Netherlands, reference number METC 2016_020\#B2016333ENG; and Kliniken der Stadt Köln gGmbH, Germany, reference number 185/2015.

The investigators have all obtained ethics approval before being allowed to conduct and participate in the study. The investigator is also responsible for fulfilling any conditions of approval imposed by the local Ethics Committee, such as regular reporting, study timing and so on.

The timeframe required for subject or personal consultee (PC) consent/ agreement is not compatible with the time sensitivity of this trial and, therefore, several approaches to obtaining informed consent/agreement will be used, all of which are consistent with the Mental Capacity Act (England; 2005) and the Declaration of Helsinki (2013). The subjects will be incapacitated at the time of eligibility (critical injury, mechanical ventilation, sedation). This study requires that the intervention to be performed rapidly, thereby necessitating that eligible patients are included in the trial very soon after hospital admission. Therefore, declaration for initial enrolment in the trial will be sought from a nominated consultee (NC), who is familiar with this study and its consenting process and is present at the trauma call. As injury is an unexpected event, it is uncommon that relatives are present at the time of hospital admission. However, if a PC for the patient is present, bearing in mind the clinical situation and their level of distress, they will be provided with brief information about the trial either verbally or in writing. If the PC objects, their wishes will be respected.

If, and when, subjects regain the physical and mental capacity to give consent, information will be provided to them and written informed consent will be sought for continuation in the trial. For any patient who was included but does not regain full capacity, agreement will be sought from a $\mathrm{PC}$ for continuation in the trial. If a patient or PC declines to give consent/ agreement for continuation at this stage, their wishes will be respected. These attempts will continue until subject consent or PC agreement is obtained. All interactions and attempts at contact with the PC and/or subject will be documented in a study consent log. In participating institutions where this is accepted according to ethics approval, patients who die before we have had the opportunity to discuss the trial and obtain consent/agreement from them or their PC, the patient will remain in the trial based on the signed declaration obtained by the NC. Should an investigator have had the opportunity to introduce themselves and discuss the trial with the PC prior to the subject's death but written agreement has not yet been obtained, then we will make a maximum of three further attempts (by any combination of telephone, Email or letter) as deemed appropriate in each individual case to contact the PC and obtain written agreement. If after these further attempts, the PC has either not been contactable or has not returned written agreement, then the subject will remain in the trial based on the NC declaration. If the PC objects to the subject remaining in the study at this stage, then their wishes will be respected.

\section{Consent for publication}

Not applicable

\section{Competing interests}

The authors declare that they have no competing interests.

\section{Publisher's Note}

Springer Nature remains neutral with regard to jurisdictional claims in published maps and institutional affiliations.

\section{Author details}

${ }^{1}$ Department of Traumatology, Oslo University Hospital, Oslo, Norway. ${ }^{2}$ Centre for Trauma Sciences, Blizard Institute, Queen Mary University of London, London, UK. ${ }^{3}$ Department of Intensive Care Medicine, Academic Medical Center, Amsterdam, The Netherlands. ${ }^{4}$ Trauma Unit, Department of Surgery, Academic Medical Center, Amsterdam, The Netherlands. ${ }^{5}$ Department for Traumatology and Orthopedic Surgery, Cologne-Merheim Medical Centre, University of Witten/Herdecke, Cologne, Germany. ${ }^{6} \mathrm{NHS}$ Blood and Transplant/Oxford University Hospital NHS Trust, John Radcliffe Hospital, Oxford, UK. ${ }^{7}$ Radcliffe Department of Medicine, University of Oxford, Oxford, UK. ${ }^{8}$ Section for Transfusion Medicine, Capital Region Blood Bank, Copenhagen University Hospital, Rigshospitalet, Copenhagen, Denmark.

Received: 16 May 2017 Accepted: 30 September 2017 Published online: 18 October 2017

\section{References}

1. Mock C. Strengthening care for the injured globally. J Trauma. 2011;70(6): 1307-16.

2. Sauaia A, Moore FA, Moore EE, et al. Epidemiology of trauma deaths: a reassessment. J Trauma. 1995;38:185-93.

3. MacLeod JBA, Lynn M, McKenney MG, Cohn SM, Murtha M. Early coagulopathy predicts mortality in trauma. J Trauma. 2003;55(1):39-44.

4. Brohi K. Acute traumatic coagulopathy. J Traume. 2003;54(6):1127-30. 
5. Frith D, Goslings JC, Gaarder C, et al. Definition and drivers of acute traumatic coagulopathy: clinical and experimental investigations. J Thromb Haemost. 2010;8(9):1919-25.

6. Hess JR, Lindell AL, Stansbury LG, Dutton RP, Scalea TM. The prevalence of abnormal results of conventional coagulation tests on admission to a trauma center. Transfusion. 2009;49(1):34-9.

7. Eastridge BJ, Malone D, Holcomb JB. Early predictors of transfusion and mortality after injury: a review of the data-based literature. J Trauma. 2006; 60(Suppl):S20-5.

8. Davenport R, Manson J, De'Ath $\mathrm{H}$, et al. Functional definition and characterization of acute traumatic coagulopathy. Crit Care. 2011;39(12):1-7.

9. Spahn DR, Cerny V, Coats TJ, Duranteau J. Management of bleeding following major trauma: a European guideline. Crit Care. 2007;11(2):414

10. Khan S, Davenport R, Raza I, Glasgow S. Damage control resuscitation using blood component therapy in standard doses has a limited effect on coagulopathy during trauma hemorrhage. Intensive Care Med. 2015;41(2):239-47.

11. Khan S, Brohi K. Hemostatic resuscitation is neither hemostatic nor resuscitative in trauma haemorrhage. J Trauma Acute Care Surg. 2014;76(3):561-8.

12. Whiting D, DiNardo JA. TEG and ROTEM: technology and clinical applications. Am J Hematol. 2014;89(2):228-32.

13. Chandler WL, Ferrell C, Trimble S, Moody S. Development of a rapid emergency hemorrhage panel. Transfusion. 2010;50:2547-52.

14. Dzik WH, Blajchman MA, Fergusson D, et al. Clinical review: Canadian National Advisory Committee on Blood and Blood Products-Massive Transfusion Consensus Conference 2011: report of the panel. Crit Care. 2011;15(6):242

15. Afshari A, Wikkelsø A, Brok J, Møller AM, Wetterlev J. Thrombelastography (TEG) or thrombelastometry (ROTEM) to monitor haemotherapy versus usual care in patients with massive transfusion. Cochrane Database Syst Rev. 2011; 16(3):CD007871.

16. Hunt H, Stanworth S, Curry N, Woolley T, Cooper C, Ukoumunne O, Zhelev Z, Hyde C. Thromboelastography (TEG) and rotational thromboelastometry (ROTEM) for trauma induced coagulopathy in adult trauma patients with bleeding. Cochrane Database Syst Rev. 2015;16(2):CD010438

17. Da Luz LT, Nascimento B, Shankarakutty AK, Rizoli S, Adhikari NK. Effect of thromboelastography (TEG $\left.{ }^{\circledast}\right)$ and rotational thromboelastometry (ROTEM $\left.{ }^{\oplus}\right)$ on diagnosis of coagulopathy, transfusion guidance and mortality in trauma: descriptive systematic review. Crit Care. 2014;18(5):518.

18. Wikkelsø A, Wetterslev J, Møller AM, Afshari A. Thromboelastography (TEG) or thromboelastometry (ROTEM) to monitor haemostatic treatment versus usual care in adults or children with bleeding. Cochrane Database Syst Rev. 2016;22(8):CD007871

19. Holcomb JB, Tilley BC, Baraniuk S, et al. Transfusion of plasma, platelets, and red blood cells in a 1:1:1 vs a 1:1:2 ratio and mortality in patients with severe trauma. JAMA. 2015;313(5):471-12

20. Johansson, et al. Hemostatic resuscitation with plasma and platelets in trauma. J Emerg Trauma Shock. 2012;5(2):120-5.

21. Roberts I, Shakur H, Coats T, Hunt B. The CRASH-2 trial: a randomised controlled trial and economic evaluation of the effects of tranexamic acid on death, vascular occlusive events and transfusion requirement in bleeding trauma patients. Health Technol Assess. 2013;17(10):1-79.

22. Maegele M, Spinella PC, Schochl $H$. The acute coagulopathy of trauma. Shock. 2012:38(5):450-8.

23. Holcomb JB, delJunco DJ, Fox EE, PROMMTT Study Group, et al. The Prospective, Observational, Multicenter, Major Trauma Transfusion (PROMMTT) study: comparative effectiveness of a time-varying treatment with competing risks. JAMA Surg. 2013;148(2):127-36.

24. Johansson PI, Sørensen AM, Larsen CF, et al. Low hemorrhage-related mortality in trauma patients in a level I trauma center employing transfusion packages and early thromboelastography-directed hemostatic resuscitation with plasma and platelets. Transfusion. 2013:53(12):3088-99.

25. Shaz BH, Dente CJ, Nicholas J, et al. Increased number of coagulation products in relationship to red blood cell products transfused improves mortality in trauma patients. Transfusion. 2010:50(2):493-500.

26. Borgman MA, Spinella PC, Perkins JG, et al. The ratio of blood products transfused affects mortality in patients receiving massive transfusions at a combat support hospital. J Trauma. 2007:63(4):805-13.

27. Rossaint R, Bouillon B, Cerny V, et al. The European guideline on management of major bleeding and coagulopathy following trauma: fourth edition. Crit Care. 2016;12(20):100.
28. Maegele M, Görlinger K. Early and individualized goal-directed therapy for trauma-induced coagulopathy. Scand J Trauma Resusc Emerg Med. 2012;20(1):15.

29. Abdelfattah K, Cripps MW. Thromboelastography and rotational thromboelastometry use in trauma. Int J Sur. 2016;33(Pt B):196-201.

30. Website European Commission FP-7 HEALTH-Contract No. F3-2013-602771: http://cordis.europa.eu/project/rcn/110071_en.html. Accessed 06 Mar 2017.
Submit your next manuscript to BioMed Central and we will help you at every step:

- We accept pre-submission inquiries

- Our selector tool helps you to find the most relevant journal

- We provide round the clock customer support

- Convenient online submission

- Thorough peer review

- Inclusion in PubMed and all major indexing services

- Maximum visibility for your research

Submit your manuscript at www.biomedcentral.com/submit
Ciomed Central 



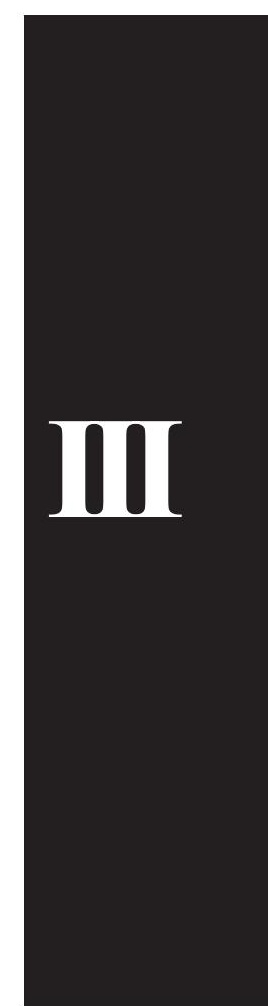





\title{
ORIGINAL
}

\section{Viscoelastic haemostatic assay augmented protocols for major trauma haemorrhage (ITACTIC): a randomized, controlled trial}

K. Baksaas-Aasen ${ }^{1}$, L. S. Gall², J. Stensballe ${ }^{3}$, N. P. Juffermans ${ }^{4}$, N. Curry ${ }^{5}$, M. Maegele ${ }^{6}$, A. Brooks $^{7}$, C. Rourke ${ }^{2}$, S. Gillespie'2, J. Murphy ${ }^{8}$, R. Maroni ${ }^{8}$, P. Vulliamy ${ }^{2}$, H. H. Henriksen ${ }^{3}$, K. Holst Pedersen ${ }^{3}$, K. M. Kolstadbraaten ${ }^{1}$, M. R. Wirtz ${ }^{4}$, D. J. B. Kleinveld ${ }^{4}$, N. Schäfer ${ }^{6}$, S. Chinna ${ }^{7}$, R. A. Davenport ${ }^{2}$, P. A. Naess ${ }^{1}$, J. C. Goslings ${ }^{4}$, S. Eaglestone ${ }^{2}$, S. Stanworth ${ }^{5,9}$, P. I. Johansson ${ }^{3}$, C. Gaarder ${ }^{1}$ and K. Brohi ${ }^{2 *}$

(C) 2020 The Author(s)

\begin{abstract}
Purpose: Contemporary trauma resuscitation prioritizes control of bleeding and uses major haemorrhage protocols (MHPs) to prevent and treat coagulopathy. We aimed to determine whether augmenting MHPs with Viscoelastic Haemostatic Assays (VHA) would improve outcomes compared to Conventional Coagulation Tests (CCTs).

Methods: This was a multi-centre, randomized controlled trial comparing outcomes in trauma patients who received empiric MHPs, augmented by either VHA or CCT-guided interventions. Primary outcome was the proportion of subjects who, at $24 \mathrm{~h}$ after injury, were alive and free of massive transfusion (10 or more red cell transfusions). Secondary outcomes included 28-day mortality. Pre-specified subgroups included patients with severe traumatic brain injury (TBI).

Results: Of 396 patients in the intention to treat analysis, 201 were allocated to VHA and 195 to CCT-guided therapy. At $24 \mathrm{~h}$, there was no difference in the proportion of patients who were alive and free of massive transfusion (VHA: 67\%, CCT: 64\%, OR 1.15, 95\% Cl 0.76-1.73). 28-day mortality was not different overall (VHA: 25\%, CCT: 28\%, OR 0.84, 95\% Cl 0.54-1.31), nor were there differences in other secondary outcomes or serious adverse events. In pre-specified subgroups, there were no differences in primary outcomes. In the pre-specified subgroup of 74 patients with TBI, 64\% were alive and free of massive transfusion at $24 \mathrm{~h}$ compared to 46\% in the CCT arm (OR 2.12, 95\% Cl 0.84-5.34).
\end{abstract}

Conclusion: There was no difference in overall outcomes between VHA- and CCT-augmented-major haemorrhage protocols.

Keywords: Trauma, Haemorrhage, Coagulopathy, Thrombelastography, Thromboelastometry 


\section{Introduction}

Major haemorrhage after trauma is estimated to be responsible for nearly half of the annual 4.6 million injury deaths worldwide [1]. Up to $50 \%$ of critically bleeding patients will die, either early from exsanguination, or later from multiple organ dysfunction or associated traumatic brain injury $[2,3]$.

Current approaches to trauma resuscitation focus on control of bleeding and management of traumainduced coagulopathy with the timely administration of haemostatic therapy [2,4]. In the initial phases of care, these therapies are often delivered empirically as part of a major haemorrhage protocol (MHP), delivering tranexamic acid, and blood components in proportions that approach the composition of whole blood [5, 6]. However, it is known that these strategies are rarely able to fully correct coagulopathy once established [7, 8], and all patients receive the same management approach, regardless of the severity or nature of their haemostatic deficits.

An alternative approach is a targeted and individualized approach to diagnosing and correcting coagulopathy. Conventional coagulation tests (CCTs) have been incorporated into algorithms but suffer from slow turnaround times and may lack the ability to guiding individual therapies [9]. We and others have investigated the diagnostic potential of point-of-care Viscoelastic Haemostatic Assays (VHAs) in trauma patients [9-15]. However, robust multi-centre and international randomized trial data on the effectiveness of VHA-guided haemostatic therapy is lacking in trauma [16-20]. Our earlier work in over 2000 trauma patients developed trauma-specific algorithms [21], and we now wished to determine whether the application of a targeted strategy in addition to standard-of-care would improve outcomes after a major trauma haemorrhage.

The "Implementing Treatment Algorithms for the Correction of Trauma-Induced Coagulopathy (ITACTIC)" trial was designed to determine the efficacy of augmenting empiric standard MHPs with VHA-guided interventions in improving outcomes from trauma haemorrhage, as compared to a standard MHP augmented by active CCT monitoring and guided interventions. We hypothesized that VHA-augmented MHPs would improve mortality and reduce the need for massive transfusion (ten or more units of red blood cell (RBC) transfusions) in the first $24 \mathrm{~h}$ after injury.

\section{Methods}

\section{Trial design}

The ITACTIC trial was a pragmatic, multi-centre, randomized controlled trial involving injured patients who

\section{Take-home message}

When standard of care is delivered with empiric balanced haemostatic therapy and intensive conventional coagulation testing, viscoelastic haemostatic assays did not improve clinical outcomes in the intention to treat cohort.

were suspected of having haemorrhage and who required at least one $\mathrm{RBC}$ transfusion. The trial compared outcomes in patients who received an empiric MHP supplemented by haemostatic therapy guided by either CCTs (CCT group) or by VHAs (VHA group). The trial was registered at ClinicalTrials.gov (NCT02593877, Nov 1st, 2015) and the protocol (Supplementary Materials) has previously been published [22].

Participating centres were members of the International Trauma Research Network (www.intrn.org) and were chosen on the basis of their ability to deliver the study design, expected enrolment rates, and the use of a standard empiric major haemorrhage protocol conforming to relevant national guidance and including balanced blood component therapy and empiric use of tranexamic acid. The trial was conducted at seven major trauma centres in Europe (Rigshospitalet (Copenhagen, Denmark), Amsterdam University Medical Centre (Amsterdam, The Netherlands), Oslo University Hospital (Oslo, Norway), Kliniken der Stadt Köln gGmbH (Cologne, Germany), The Royal London Hospital (London, UK), John Radcliffe Hospital (Oxford, UK) and Nottingham University Hospitals, Queens Medical Centre (Nottingham, UK)). The VHA device used at each study site (Thromboelastography-TEG 6S Haemostasis Analyzer, Haemonetics ${ }^{\circledR}$ Corporation, or Rotational Thromboelastometry-ROTEM ${ }^{\circledR}$ Sigma, TEM Innovations $\mathrm{GmbH}$ ) was determined by existing familiarity with a specific device appliance and to ensure a balanced use of the devices across the study.

The trial was designed by the authors, and the design was approved by the trial sponsor (Queen Mary University of London) and the national research ethics committees of participating institutions. Clinical trial oversight, monitoring and data management were conducted by the clinical studies unit of the Centre for Trauma Sciences, Queen Mary University of London. An independent Data Monitoring Committee and a Trial Steering Committee provided oversight of trial activities.

\section{Patient population}

Adult trauma patients were enrolled if they presented with clinical signs of bleeding activating the local MHP and if RBC transfusion had been initiated. Participants had to be randomized within $3 \mathrm{~h}$ of injury and maximum 
of $1 \mathrm{~h}$ after admission to the emergency department. There were no additional exclusion criteria.

Consent for initial enrolment in the trial was provided on behalf of incapacitated patients by a nominated consultee, usually an independent clinician in charge of patient care. Subsequent consent was given by a personal consultee (e.g. a family member) when identified and able to go through the informed consent process. Individual informed consent was subsequently obtained if the patient regained the physical and mental capacity to provide such consent. If the patient died or was otherwise unable to give consent, previous nominated professional or personal consultee consent remained in effect. Patients who subsequently withdrew their consent were removed from the trial.

\section{Randomization and blinding}

Enrolled patients were randomized in a 1:1 ratio to the VHA or the CCT groups, with block randomization by the centre. Randomization codes were generated and secured by an independent statistician. Block size was unknown to on-site study teams. Group allocation was by study personnel opening the numbered opaque sealed envelope in sequence taken from a stack held by each study site. Once an envelope had been opened, the subject was considered enrolled. The trial was un-blinded to the treating clinical teams, while research personnel collecting subsequent safety and outcome data were blinded to group allocation.

\section{Procedures}

All patients received their local hospital's standard MHP, based on the empiric delivery of tranexamic acid; blood components delivered in a 1:1:1 ratio of $\mathrm{RBCs}$, plasma and platelet transfusions; and limited infusion of crystalloid fluids. In both groups, blood was drawn for coagulation analysis at baseline and after every four units of RBCs transfused, until haemostasis. In the CCT group, these were the conventional coagulation tests performed in the laboratory, while in the VHA assays, analyses were performed at the point of care. Haemostatic therapy was delivered based on results of these according to the TACTIC algorithms [21], which define triggers for additional administration of platelet, fibrinogen, plasma and antifibrinolytic therapies.

\section{Definitions}

The time of haemostasis was defined as $1 \mathrm{~h}$ after the last $\mathrm{RBC}$ transfusion was given and the treating clinicians stated haemostasis had been achieved. Massive transfusion was defined as the administration of ten or more units of RBCs in the first $24 \mathrm{~h}$ after injury. Severe traumatic brain injury (TBI) was defined as an Abbreviated
Injury Scale score in the brain of four, five, or six [23]. We used an anatomical head injury score rather than an assessment of admission level of consciousness as the latter is affected by the depth of shock in this group of patients. We used prothrombin time ratio (PTr) as a test of coagulation available in both arms of the trial [24] and defined PTr $>1.2$ as abnormal. For reporting blood component therapy across study centres, one pool of cryoprecipitate was considered equivalent to two grams of fibrinogen concentrates (or fibrinogen equivalent dose in grams); and platelet concentrates are reported as administered in pooled components (one pool = four individual platelet units).

\section{Outcomes}

The primary outcome of this study was the proportion of subjects who, at $24 \mathrm{~h}$ after injury, were alive and free of massive transfusion. Secondary endpoints included: all-cause mortality at $6 \mathrm{~h}, 24 \mathrm{~h}, 28$ days, and 90 days post-admission; total blood components; 28-day ventilator-free, and intensive care unit (ICU)-free days; total hospital length-of-stay; and the proportion of patients with symptomatic thromboembolic events, with multiple organ dysfunction, and with serious adverse events.

\section{Statistical analysis}

The planned sample size for this study was 392, 196 in each study arm. Prior data from the study centres estimated that approximately $28 \%$ of patients die or receive a massive transfusion by $24 \mathrm{~h}$. Based on an estimated reduction in this proportion to $15 \%$ in the VHA group, and with a significance level of 0.05 using a two-sided test, 170 patients per group were required to achieve $80 \%$ power, increasing to 196 per group to account for a predicted drop-out rate of $15 \%$.

All primary and secondary outcomes were analysed as intention-to-treat and per-protocol. The per-protocol group was defined excluding patients who did not have at least one VHA or CCT test performed, who did not meet the inclusion criteria, who received the wrong test, who died within $60 \mathrm{~min}$ after baseline blood sampling or who achieved haemostasis within $60 \mathrm{~min}$ of baseline sampling.

The primary endpoint was assessed by logistic regression to produce an odds ratio with $95 \%$ confidence interval. Mortality was compared between the two arms as a binary outcome by logistic regression and using KaplanMeier methods and the log-rank test. Time to haemostasis, 28-day ventilator-free and ICU-free days, total hospital length-of-stay, EQ-5D quality-of-life scores, total number of blood products transfused, numbers receiving study intervention (post-hoc analysis) and time to intervention (post-hoc analysis) were compared using 
the Wilcoxon-Mann-Whitney or Chi-square tests. Missing data for the primary outcome were not expected. For secondary outcomes, data were assumed to be missing at random, therefore participants with missing data for a measure were excluded from any statistical comparisons regarding that measure. Denominators for analyses are presented throughout.

Pre-specified subgroups of interest were patients with and without severe TBI (defined as having an injury to the head of Abbreviated Injury Scale score of 4 or more); patients with a prolonged PTr at baseline; patients receiving a massive transfusion; and patients on prior oral anticoagulant therapies. Stratification within subgroups was not possible as laboratory and imaging results were not available at the time of randomization and enrolment. The pre-defined subgroups were analysed by intention-to-treat for the primary outcome (except for the patients receiving a massive transfusion as not applicable) and some secondary outcomes (posthoc analyses), such as 28-day mortality, total number of blood products, numbers receiving study intervention and time to intervention. As a comparison with the opposite subgroup, patients without a prolonged PTr were also analysed (post-hoc analysis). Interaction p-values were calculated (post-hoc analysis) to inspect the effect of each subgroup on the outcome of interest. The primary endpoint and 28-day mortality analyses for the severe TBI subgroup were also performed adding covariates to the logistic regressions to adjust for any risk differences between study arms at baseline (post-hoc analysis). Statistical analyses were performed using Stata version 13. All applied tests were two-sided and p-values of less than 0.05 were considered statistically significant. A pre-defined interim analysis was performed after the enrolment of 100 patients and the Data Monitoring Committee reviewed all data on outcomes for every 50 patients enrolled. The statistical analyses plan is presented in the Supplementary Materials.

\section{Results}

From 1st of June 2016 to 30th of July 2018, 480 trauma patients were eligible for enrolment, of which 411 were randomized. 15 patients subsequently withdrew consent, leaving 396 subjects for the intention-to-treat analysis (Fig. 1): 201 patients were allocated to the VHA group and 195 to the CCT group. Baseline characteristics were well matched between treatment groups (Table 1). Two-thirds of the intention-to-treat cohort had sustained blunt trauma alone, and the overall median Injury Severity Score (ISS) was 26 (interquartile range (IQR) 17-36, with a score over 15 indicating severe trauma). The median time from injury to admission was $69 \mathrm{~min}$ (IQR 45-96 $\mathrm{min}$ ). At enrolment, participants had already received a median of two units of RBCs (IQR 1-4) and 9 had already received massive transfusion (ten or more units of RBCs). Of the 377 patients who received tranexamic acid, 268 (71\%) received the $1 \mathrm{~g}$ bolus prior to enrolment.

After enrolment and before haemostasis, 120/178 (67\%) patients in the VHA group received a study intervention, compared to $62 / 170(36 \%)$ in the CCT group (Supplementary Table S1). The study interventions were given a median of 21 min earlier in the VHA group (VHA $61 \mathrm{~min}$, IQR 48-85; CCT $80 \mathrm{~min}$, IQR 60-106, Supplementary Table S1), with 89/139 (64\%) of patients in the VHA group receiving the first study intervention within $3 \mathrm{~h}$ of injury compared to $45 \%$ $(39 / 86)$ in the CCT group. Between baseline and haemostasis, patients received a median of three units of RBCs and four units of plasma in both arms of the trial (Supplementary Table S1). Within this period, patients in the VHA arm received more fibrinogen supplementation (median fibrinogen equivalent dose-VHA: $4 \mathrm{~g}$, IQR 0-4; CCT: 0 g, IQR 0-4 Supplementary Table S1).

At $24 \mathrm{~h}$ after injury, there was no difference between the two groups in the proportion of patients who were alive and free of massive transfusion (VHA 67\%, CCT $64 \%$, OR 1.15, 95\% CI 0.76-1.73, Fig. 2). In the VHA arm, 29/201 (14\%) of patients had died and 53/201 (26\%) had received a massive transfusion at $24 \mathrm{~h}$, while in the CCT arm, 33/195 (17\%) of patients had died and 55/195 (28\%) had received a massive transfusion (Table 2). At 28 days, 50/201 (25\%) of patients in the VHA arm and 55/194 (28\%) patients in the CCT arm had died (OR 0.84, 95\% CI 0.54-1.31, Table 2 and Supplementary Figure S1). This pattern persisted to 90 days (Fig. 3).

There were no statistically significant differences in other secondary outcomes between the two study groups, including the rate of multiple organ dysfunction, the incidence of symptomatic thromboembolic events, and a number of ventilator-free days or ICU-free days at day 28 (Table 2). Hospital length-of-stay was also similar in both groups and so were quality-of-life (EQ-5D) scores at both discharge/28 days and 90 days (Table 2). Safety and cause-of-death profiles were similar across the two groups (Table 3).

For the per-protocol analysis, 83 patients were excluded from the ITT cohort: five for not meeting the entry criteria, eight for receiving the wrong treatment, and 54 for reaching haemostasis and 16 for dying within the first hour (Fig. 1). This left 313 patients in the perprotocol cohort (150 VHA, 163 CCT, Fig. 1). There was no statistically significant difference between study arms for the primary outcome or for 28-day mortality in the per-protocol group (Fig. 2 and Supplementary Fig. S1). 


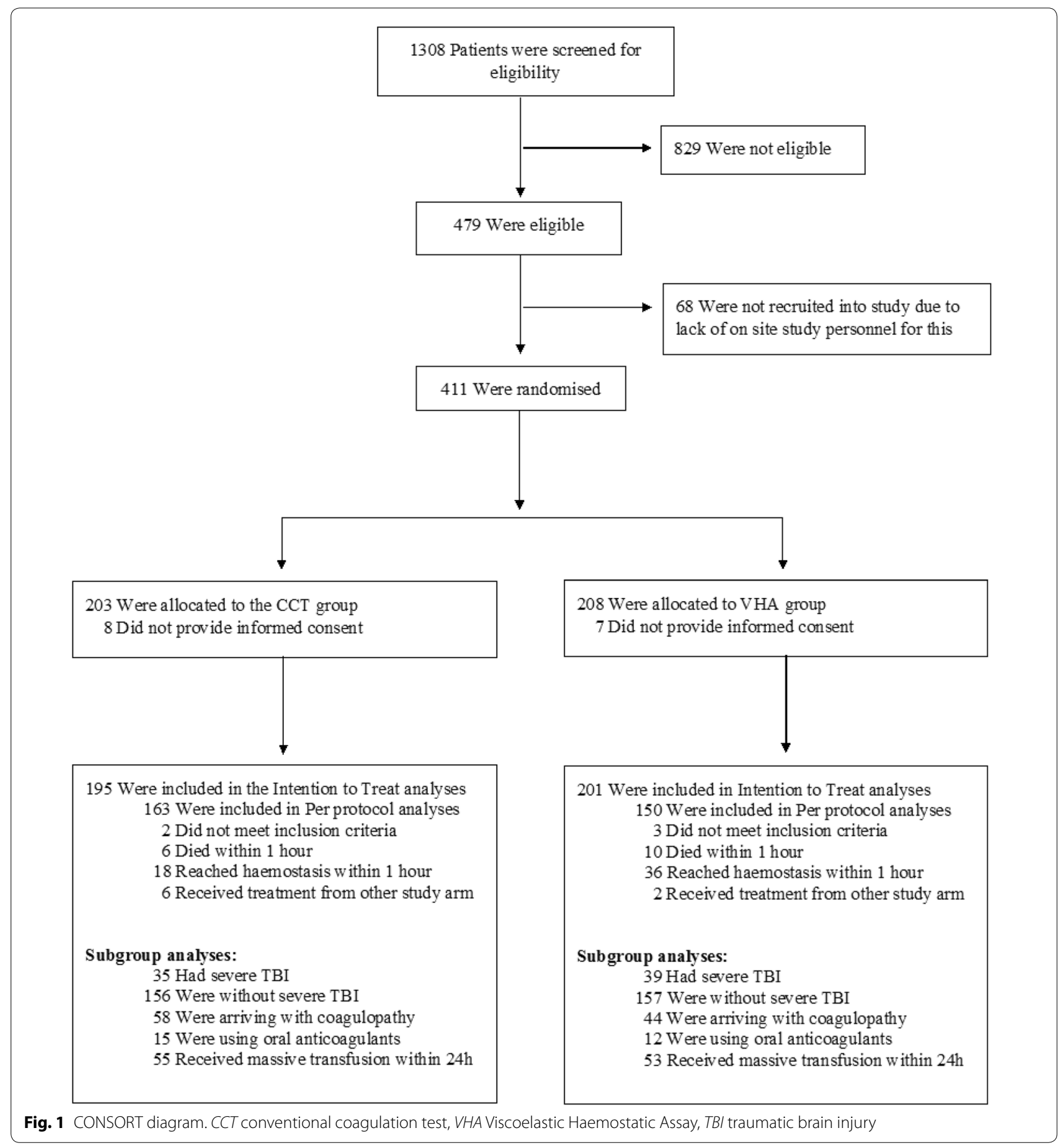

Across the pre-specified subgroups, there was no statistically significant difference in the primary outcome (Fig. 2). In the subgroup of 74 patients who presented with severe TBI, 25/49 (64\%) patients in the VHA arm were alive and free of massive transfusion at $24 \mathrm{~h}$, compared to $16 / 35(46 \%)$ in the CCT arm (OR 2.12 95\% CI 0.84-5.34). After risk adjustment for admission characteristics, this odds ratio was unchanged at 2.10 (95\% CI 0.51-8.61, Supplemental Table S3). Additional analyses on secondary outcomes of the TBI and other subgroups are presented in the Supplementary Materials (Fig. S1, Tables S1-S3). 
Table 1 Demographics, injuries and admission characteristics and initial therapies (intention-to-treat population)

\begin{tabular}{|c|c|c|}
\hline & $\mathrm{CCT}(n=195)$ & VHA $(n=201)$ \\
\hline Median age (IQR) & $43(28-59), n=194$ & $40(26-54), n=197$ \\
\hline Male sex - no. (\%) & 159/194 (82\%) & 145/198 (73\%) \\
\hline Prior oral anticoagulation - no. (\%) & 15/192 (8\%) & $12 / 198(6 \%)$ \\
\hline \multicolumn{3}{|l|}{ Injuries \& Admission Physiology } \\
\hline Injury caused by blunt trauma alone^ - no. (\%) & 130/194 (67\%) & 133/198 (66\%) \\
\hline Median Injury Severity Score (IQR) & $26(16-35), n=191$ & $26(17-37), n=196$ \\
\hline Severe TBI (AIS Head > 3) - no. (\%) & 35/191 (18\%) & $39 / 201(20 \%)$ \\
\hline Median time from injury to admission (IQR) - min & $67(42-98), n=191$ & $70(48-95), n=195$ \\
\hline Median heart rate (IQR) - beats/min & $105(82-123), n=181$ & $103(87-127), n=190$ \\
\hline Median systolic blood pressure (IQR) - $\mathrm{mmHg}$ & $90(74-110), n=170$ & $95(73-120), n=178$ \\
\hline Median Glasgow Coma Scale score (IQR) & $13(3-15), n=191$ & $12(3-15), n=194$ \\
\hline Patients with PTr $>1.2$ - no. (\%) & $58 / 181(32 \%)$ & $44 / 175(25 \%)$ \\
\hline Median base deficit (IQR) - mEq/L & $7.2(4.2-13), n=188$ & $8.2(4.4-12.8), n=190$ \\
\hline Median lactate (IQR) - mEq/L & $4.4(2.8-8.2), n=185$ & $4.5(2.9-8), n=191$ \\
\hline Median fibrinogen level (IQR) - g/L & $2.0(1.4-2.4), n=n=177$ & $1.9(1.5-2.4), n=166$ \\
\hline \multicolumn{3}{|l|}{ Pre-baseline therapy } \\
\hline Received tranexamic acid bolus—no. (\%) & 190/193 (98\%) & 187/200 (94\%) \\
\hline Median units of RBCs (IQR) & $2(1-4), n=179$ & $2(1-4), n=n=187$ \\
\hline Median units of FFP/Octaplasma (IQR) & $0(0-2), n=179$ & $0(0-2), n=187$ \\
\hline Median equivalent dose of fibrinogen (IQR) - $g$ & $0(0-0), n=184$ & $0(0-0), n=185$ \\
\hline Median units of Platelets (IQR) & $0(0-0), n=179$ & $0(0-0), n=n=187$ \\
\hline
\end{tabular}

$C C T$ conventional coagulation test, $V H A$ viscoelastic haemostatic assay, IQR interquartile range, $T B I$ traumatic brain injury, AIS abbreviated injury scale, $P T r$ prothrombin time ratio, $R B C$ red blood cell, FFP fresh frozen plasma

$\wedge$ The other categorisations used for a type of trauma were: penetrating; or blunt and penetrating combined

\section{Discussion}

Our international randomized trial has evaluated the role of VHAs to individualize care and augment empiric haemostatic resuscitation in the challenging and timecritical environment of trauma care. Empiric haemostatic therapy was delivered in both arms of the trial, with balanced blood component transfusions, use of tranexamic acid and restricted crystalloid infusions. Intensive coagulation monitoring was also performed in both arms after every four units of red cell transfusions, with additional haemostatic therapies delivered based on these results. We saw no difference overall in primary or secondary outcomes between CCT and VHA-guided haemostatic therapy.

While this study aimed to explore the effect of VHAdirected assessment and treatment of coagulopathy in trauma patients, the overall prevalence of coagulopathy was lower than we expected. Nearly three quarters of the patients were not coagulopathic (by PTr) at baseline and very few of these patients subsequently developed a prolonged PTr before haemostasis. These lower rates are likely due to the effect of optimisation of balanced haemostatic therapy with early prehospital and in-hospital use of transfusions prior to randomisation and during haemorrhage. This alone was not sufficient to prevent trauma-induced coagulopathy, as coagulation deficits identified by CCTs resulted in treatment in $36 \%$ of patients in this arm of the trial (Supplementary Table S1). A previous single-centre trauma study reported a larger benefit with VHA-directed therapy, but subjects in the control arm received less empiric haemostatic transfusion therapy and the role of CCT monitoring during bleeding was not reported [25]. For those patients who never developed a coagulopathy, it is unsurprising that coagulation monitoring did not alter the clinical outcome. The relatively high numbers of these patients in our study reduced our ability to detect a difference in clinical outcomes.

Despite the delivery of optimized and individualised standard of care in both cohorts, $67 \%$ of patients in the VHA-augmented cohort received study interventions, 1.8 times more than in the CCT group. This indicates the widespread occurrence of coagulation deficits which were not detected by the CCTs. We are unable to ascertain in this analysis if these deficits were corrected by the directed therapies or whether the doses prescribed within the algorithms were too low. 


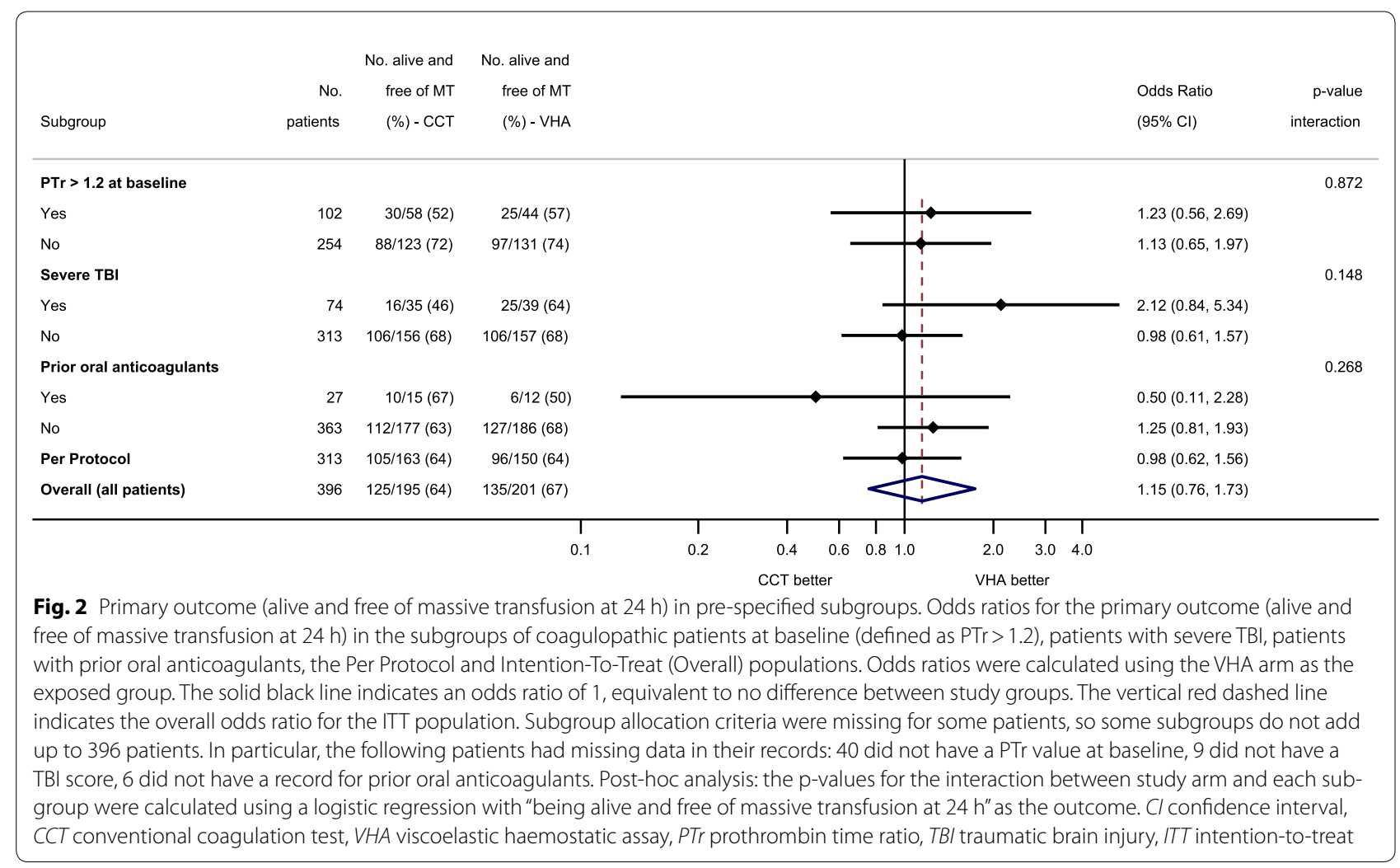

The reduction in 28-day mortality in the VHA group observed in patients who also had severe TBI was unexpected and may have been a chance finding. However, if borne out in future work, it potentially represents an important opportunity for improving outcomes in trauma care [26]. TBI is responsible for at least 25\% of deaths in critically bleeding patients, and $50 \%$ of all trauma deaths [2]. Most previous clinical trials in trauma haemorrhage have excluded severe brain injuries as their outcomes were felt to be immutable by changes in haemostatic management. There is a small but growing body of evidence that directed coagulopathy management may reduce brain injury mortality, such as the two recent trials of tranexamic acid for TBI $[27,28]$. Correction of coagulopathy may act to reduce intracerebral bleeding; decrease cerebral ischaemia due to reduced depth and duration of haemorrhagic shock; and/or reduce cerebral inflammation via crosstalk mechanisms, all of which constitute important areas for future brain injury research. The ITACTIC results are consistent with these hypotheses, and suggests this is an area for research in a field which has mostly focused on cerebral oxygenation and perfusion, and where there has been little change in practice or improvement in outcomes for some time [29-32].

This study was limited in a number of ways, including the lower observed difference in effect size in the primary endpoint between the two cohorts than originally predicted (observed: 3\%, predicted: 13\%). Empiric delivery of balanced transfusion and haemostatic therapies prior to randomisation and during haemorrhage likely contributed to this. Another caveat is that we did not adjust our secondary endpoint and post-hoc analyses for multiple comparisons due to the preponderance of non-significant results. As viscoelastic testing was only performed in the VHA group, we could not identify all coagulopathic patients and could not explore the effect of targeted therapies in coagulation deficits that were only detectable by VHA. Interpretation of the results from our subgroups is also limited by lack of group allocation stratification, which was impossible with this study design, as neither coagulation status nor the presence of severe brain injury could be known prior to randomisation. The study was conducted at large major trauma centres, with experience of the use of VHA devices in a research setting, although most did not have VHA-guided care in routine clinical use at the start of the trial. The study was challenging to deliver on many levels and the experience in other centres may be different.

When standard care is delivered to bleeding trauma patients, with empiric balanced transfusion therapy and intensive CCT monitoring, VHAs identify more coagulation deficits and deliver additional haemostatic 
Table 2 Secondary outcomes for the intention-to-treat population

\begin{tabular}{|c|c|c|c|c|}
\hline & $\mathrm{CCT}(n=195)$ & VHA $(n=201)$ & Odds ratio $(95 \% \mathrm{Cl})$ & $p$ value \\
\hline Mortality at $6 \mathrm{~h}-\mathrm{no} .(\%)$ & $22 / 195(11 \%)$ & $22 / 201(11 \%)$ & $0.97(0.52-1.80)$ & 0.915 \\
\hline Mortality at 24 h-no. (\%) & $33 / 195(17 \%)$ & $29 / 201(14 \%)$ & $0.83(0.48-1.42)$ & 0.495 \\
\hline Mortality at 28 days-no. (\%) & $55 / 194(28 \%)$ & $50 / 201(25 \%)$ & $0.84(0.54-1.31)$ & 0.435 \\
\hline Mortality at 90 days—no. (\%) & $56 / 177(31 \%)$ & $53 / 179(29 \%)$ & $0.91(0.58-1.42)$ & 0.678 \\
\hline Death from exsanguination — no. (\%) & $17 / 56(30 \%)$ & $13 / 51(25 \%)$ & $0.78(0.34-1.82)$ & 0.576 \\
\hline Died before haemostasis—no. (\%) & $24 / 54(44 \%)$ & $19 / 50(38 \%)$ & $0.77(0.35-1.67)$ & 0.505 \\
\hline Median time to haemostasis^ (IQR)—mins & $122(80-185), n=170$ & $125(77-185), n=176$ & & 0.929 \\
\hline PTr $>1.2$ at haemostasis^—no. (\%) & $17 / 151(11 \%)$ & $21 / 142(15 \%)$ & $1.37(0.70-2.69)$ & 0.369 \\
\hline Massive transfusion at $24 \mathrm{~h}-\mathrm{no}$. (\%) & $55 / 195(28 \%)$ & $53 / 201(26 \%)$ & $0.91(0.59-1.42)$ & 0.682 \\
\hline Patients with symptomatic TE^^—no. (\%) & $27 / 195(14 \%)$ & $17 / 201(9 \%)$ & $0.57(0.31-1.08)$ & 0.088 \\
\hline Patients with MODS^^^——no. (\%) & 134/159 (84\%) & $141 / 164(86 \%)$ & $1.14(0.62-2.10)$ & 0.668 \\
\hline Median 28-day ventilator-free days (IQR) & $20(0-26), n=192$ & $17(0-25), n=198$ & & 0.422 \\
\hline Median 28-day ICU-free days (IQR) & $15(0-23), n=192$ & $13(0-23), n=198$ & & 0.691 \\
\hline Median hospital LOS in survivors (IQR) & $24(10-42), n=138$ & $29(13-49), n=147$ & & 0.147 \\
\hline Median EQ-5D $\wedge \wedge \wedge$ index at discharge/28 days (IQR) & $49(25-60), n=86$ & $40(28-60), n=n=92$ & & 0.672 \\
\hline 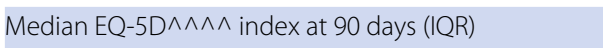 & $60(40-70), n=75$ & $53(40-70), n=72$ & & 0.718 \\
\hline
\end{tabular}

Proportions were calculated excluding any missing records

CCT conventional coagulation test, VHA viscoelastic haemostatic assay, $\mathrm{Cl}$ confidence interval, $P T r$ prothrombin time ratio, TE thromboembolic events, $M O D S$ multiple organ dysfunction syndrome, ICU intensive care unit, LOS length of stay

$\wedge$ Patients with haemostasis (CCT: $n=n=170, \mathrm{VHA}: n=178)$

$\wedge \wedge$ Myocardial infarction and Embolic strokes are included in thromboembolic events

$\wedge \wedge \wedge$ Multiple Organ Dysfunction Syndrome (MODS) defined as having a Sequential Organ Failure Assessment score of 6 or more on a day

$\wedge \wedge \wedge \wedge E Q-5 D$ is a questionnaire by EuroQol used as a measure of overall health status

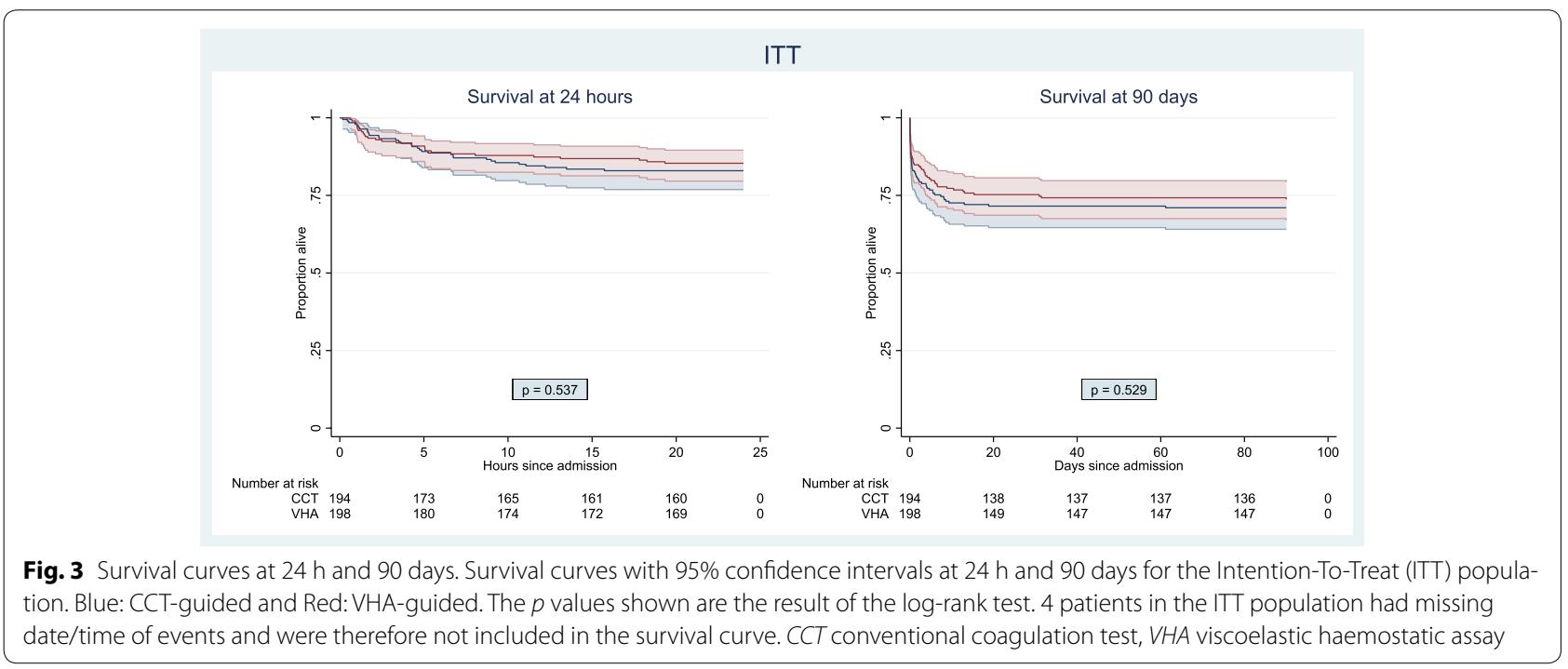


Table 3 Distribution of serious adverse events (SAEs) and causes of death

\begin{tabular}{|c|c|c|c|c|}
\hline \multirow[t]{2}{*}{ SAE description^^} & \multicolumn{2}{|l|}{$\begin{array}{l}\mathrm{CCT} \\
(n=195)\end{array}$} & \multicolumn{2}{|l|}{$\begin{array}{l}\text { VHA } \\
(n=201)\end{array}$} \\
\hline & Events & Patients & Events & Patients \\
\hline Infection & $34(34 \%)$ & $30(32 \%)$ & $35(31 \%)$ & $29(29 \%)$ \\
\hline Thromboembolic^^^ & $24(24 \%)$ & $22(24 \%)$ & $16(14 \%)$ & $15(15 \%)$ \\
\hline Ischemic & $0(0 \%)$ & $0(0 \%)$ & $6(5 \%)$ & $6(6 \%)$ \\
\hline Organ failure & $5(5 \%)$ & $5(5 \%)$ & $9(8 \%)$ & $9(9 \%)$ \\
\hline Acute kidney injury & $6(6 \%)$ & $6(6 \%)$ & $6(5 \%)$ & $6(6 \%)$ \\
\hline Acute lung injury & $6(6 \%)$ & $5(5 \%)$ & $12(11 \%)$ & $8(8 \%)$ \\
\hline New onset major bleeding & $9(9 \%)$ & $9(10 \%)$ & $6(5 \%)$ & $6(6 \%)$ \\
\hline Cardiac & $6(6 \%)$ & $6(6 \%)$ & $10(9 \%)$ & $10(10 \%)$ \\
\hline Neurological & $0(0 \%)$ & $0(0 \%)$ & $4(4 \%)$ & $4(4 \%)$ \\
\hline Other & $11(11 \%)$ & $10(11 \%)$ & $8(7 \%)$ & $8(8 \%)$ \\
\hline Total & $101(100 \%)$ & $93(100 \%)$ & $112(100 \%)$ & $101(100 \%)$ \\
\hline Cause of Death & & Patients & & Patients \\
\hline Uncontrolled Bleeding & & $17(30 \%)$ & & $13(25 \%)$ \\
\hline Head Injury & & $19(34 \%)$ & & $16(30 \%)$ \\
\hline Multiple Organ Dysfunction & & $6(11 \%)$ & & $10(19 \%)$ \\
\hline Thromboembolism & & $0(0 \%)$ & & $2(4 \%)$ \\
\hline Multiple Injuries & & $4(7 \%)$ & & $1(2 \%)$ \\
\hline Other & & $10(18 \%)$ & & $9(17 \%)$ \\
\hline Unknown & & $0(0 \%)$ & & $2(4 \%)$ \\
\hline Total & & $56(100 \%)$ & & $53(100 \%)$ \\
\hline
\end{tabular}

Serious Adverse Events (SAEs) were defined as any adverse event, adverse transfusion reaction or unexpected adverse transfusion reaction that resulted in death, was life-threatening, required hospitalization or prolongation of existing hospitalization, resulted in persistent or significant disability or incapacity, resulted in a congenital anomaly/birth defect or other medically significant event

$\wedge$ In the event field, SAEs experienced multiple times by the same patient are included in the count. In the patients field, patients experiencing multiple SAEs of the same type are included only once per type of SAE

$\wedge \wedge$ Myocardial infarction and embolic stroke are included in thromboembolic SAEs

interventions. However, all patients do not benefit from this approach and further research is required to identify injury types and physiologies that may benefit from this approach. Additional analyses should also explore the coagulation deficits identified by VHA alone, and the response of the coagulation system to the algorithm-prescribed haemostatic agents.

\section{Electronic supplementary material}

The online version of this article (https://doi.org/10.1007/s00134-020-06266-1) contains supplementary material, which is available to authorized users.

\footnotetext{
Author details

${ }^{1}$ Oslo University Hospital \& University of Oslo, Oslo, Norway. ${ }^{2}$ Centre for Trauma Sciences, Queen Mary University of London, Blizard Institute, 4 Newark Street, London E1 2AT, UK. ${ }^{3}$ Copenhagen University Hospital, Rigshospitalet, Copenhagen, Denmark. ${ }^{4}$ Amsterdam University Medical Centres, Amsterdam, The Netherlands. ${ }^{5}$ Oxford University Hospital NHS Trust, Oxford, UK. ${ }^{6}$ Cologne-Merheim Medical Centre, University of Witten/Herdecke, Cologne, Germany. ${ }^{7}$ Nottingham University Hospitals NHS Trust, Nottingham, UK. ${ }^{8}$ Queen Mary University of London, London, UK. ${ }^{9}$ NHS Blood and Transplant, Bristol, UK.
}

\section{Acknowledgements}

We would like to thank all the patients and their families who participated in this project. We are grateful for the contributions to the study of the Trial Steering Committee: Beverley Hunt (Chair), Heidi Doughty, Louis Riddez, Leo Geeraedts, Alison Rogers; and the Data Monitoring Committee: Ravi Gill (Chair), Jan Jansen and Phil Edwards.We would also like to acknowledge Joao Dias and Jan Hartmann (Haemonetics ${ }^{\circledR}$ Corporation) and Klaus Görlinger (TEM International $\mathrm{GmbH}$ ) for their personal support for the entire TACTIC program. We also acknowledge Amar Ahmed for early statistical support to the trial.This study is funded by the European Commission under the FP-7 HEALTH-Contract No. F3-2013-602771, entitled "Targeted Action for Curing Trauma Induced Coagulopathy" (TACTIC).Website European Commission FP-7 HEALTH-Contract No. F3-2013-602771: http://cordis.europa.eu/project/ rcn/110071_en.html.

\section{Author contributions}

Study design, development and study set up were performed by LSG, KB-A, JS, NPJ, NC, MM, CR, PV, RAD, PAN, JCG, SE, SS, PIJ, CG, KB. Site set up, subject enrolment, data collection and research governance were performed by KB-A, LSG, JS, NPJ, NC, MM, CR, PV, RAD, PAN, JCG, SE, SS, PIJ, CG and KB. RM and JM performed the statistical analysis. Initial draft of the manuscript was by KB-A $\mathrm{RM}$ and $\mathrm{KB}$. All authors reviewed and commented on this and subsequent versions of the manuscript. AB, SC, SG, HHH, KHP, KMK, MRW, DJBK and NS contributed in data collection and commented on the manuscript. All authors vouch for the accuracy and completeness of the data and analyses, and for the fidelity of the trial to the protocol. No one who is not an author participated in the writing or editing of the manuscript. 


\section{Funding}

The study was part of the "Targeted Action for Curing Trauma-Induced Coagulopathy" (TACTIC) program, funded by the European Commission under the FP7 framework (Grant No. F3-2013-602771). Both TEM ${ }^{\circledR}$ International GmbH and Haemonetics ${ }^{\circledR}$ Corporation were collaborating organizations in the TACTIC program. For this ITACTIC study, they provided the ROTEM Sigma and TEG $6 \mathrm{~S}$ analyzers and all reagents to the relevant participating institutions. Neither these organizations nor any of their representatives had any role in the study design, data collection and analysis, decision to publish, or preparation of the manuscript.

\section{Compliance with ethical standards}

\section{Conflicts of interest}

K.Baksaas-Aasen, L.S.Gall, J.Stensballe, N.P.Juffermans, N.Curry, C.Rourke, S.Gillespie, J.Murphy, R.Maroni, P.Vulliamy, H.H.Henriksen, K.Holst Pedersen, K.M. Kolstadbraaten, M.R.Wirtz, D.J.B. Kleinveld, N.Schäfer, S.Chinna, P.A.Naess, J.C.Goslings, S.Eaglestone and S.Stanworth declare no conflicts of interest. M.Maegele has received honoraria for lectures and speakers' bureaus, congress travel support as well as financial support for research projects from Astra Zeneca, Bayer, CSL Behring, IL-Werfen/TEM International, LFB Biomedicaments and Portola Inc. A. Brooks has received a research grant from Haemonetics Corp. in the form of cartridges and reagent support. He has also served on advisory panel for Haemonetics Corp. and TEM International and received honoraria for education lectures for Johnson and Johnson. P.I. Johansson's has received unrestricted research grants from Haemonetics Corp. and Octapharma AG. C.Gaarder has received honoraria for lectures from Octapharma and research grant support from Haemonetics and TEM International in the form of device and reagent support. She has also previously served on the advisory board for Nycomed. K.Brohi and R.Davenport have received research grant support from TEM International in the form of device and reagent support. K.Brohi has previously served on external advisory panels for Haemonetics Corp, TEM International, CSL Behring, Bayer and Astra Zeneca.

\section{Ethical approval}

The study was designed by the authors, and the design was approved by the trial sponsor (Queen Mary University of London) and the national research ethics committees of participating institutions. The study was in accordance with the 1964 Helsinki Declaration.

\section{Consent to participate}

Initial enrolment in the trial was provided on behalf of incapacitated patients by a personal or nominated consultee until the patient regained the physical and mental capacity to provide such consent.

\section{Availability of data and material}

De-identified individual participant data underlying primary and secondary endpoints will be available on request from researchers, after approval of proposal. Study protocol and statistical analysis plan are presented in the Supplementary Materials, and informed consent form will be shared, if requested. The data will be available by contacting the corresponding author after publication.

\section{Open Access}

This article is licensed under a Creative Commons Attribution-NonCommercia 4.0 International License, which permits any non-commercial use, sharing, adaptation, distribution and reproduction in any medium or format, as long as you give appropriate credit to the original author(s) and the source, provide a link to the Creative Commons licence, and indicate if changes were made. The images or other third party material in this article are included in the article's Creative Commons licence, unless indicated otherwise in a credit line to the material. If material is not included in the article's Creative Commons licence and your intended use is not permitted by statutory regulation or exceeds the permitted use, you will need to obtain permission directly from the copyright holder. To view a copy of this licence, visit http://creativecommons.org/licen ses/by-nc/4.0/.

\section{Publisher's Note}

Springer Nature remains neutral with regard to jurisdictional claims in published maps and institutional affiliations.
Received: 16 February 2020 Accepted: 20 September 2020

Published online: 13 October 2020

\section{References}

1. Gruen RL, Brohi K, Schreiber M et al (2012) Haemorrhage control in severely injured patients. Lancet 380:1099-1108

2. Cole E, Weaver A, Gall L et al (2019) A decade of damage control resuscitation: new transfusion practice, new survivors, new directions. Ann Surg. https://doi.org/10.1097/SLA.0000000000003657

3. Brohi K, Gruen RL, Holcomb JB (2019) Why are bleeding trauma patients still dying? Intensive Care Med 45:709-711

4. CRASH-2 collaborators (2011) The importance of early treatment with tranexamic acid in bleeding trauma patients: an exploratory analysis of the CRASH-2 randomised controlled trial. Lancet 377:1096-1101

5. NICE recommendations Major Trauma (NG39) published February 2016. https://www.nice.org.uk/guidance/ng39. Accessed 1st of February 2020

6. Holcomb JB, Tilley BC, Baraniuk S et al (2015) Transfusion of plasma, platelets, and red blood cells in a 1:1:1 vs a 1:1:2 ratio and mortality in patients with severe trauma. PROPPR Rand Clin Trial JAMA 313:471-482

7. Khan S, Davenport R, Raza I et al (2015) Damage control resuscitation using blood component therapy in standard doses has a limited effect on coagulopathy during trauma haemorrhage. Intensive Care Med 41:239-247

8. Balvers K, van Dieren S, Baksaas-Aasen K et al (2017) Combined effect of therapeutic strategies for bleeding injury on early survival, transfusion needs and correction of coagulopathy. Br J Surg 104:222-229

9. Davenport R, Manson J, De'Ath $\mathrm{H}$ et al (2011) Functional definition and characterization of acute traumatic coagulopathy. Crit Care Med 39:2652-2658

10. Johansson PI, Sørensen AM, Larsen CF et al (2013) Low haemorrhagerelated mortality in trauma patients in a Level I trauma centre employing transfusion packages and early thromboelastography-directed haemostatic resuscitation with plasma and platelets. Transfusion 53:3088-3099

11. Hagemo JS, Christiaans SC, Stanworth SJ et al (2015) Detection of acute traumatic coagulopathy and massive transfusion requirements by means of rotational thromboelastometry: an international prospective validation study. Crit Care 19:97

12. Holcomb JB, Minei KM, Scerbo ML et al (2012) Admission rapid thrombelastography can replace conventional coagulation tests in the emergency department. Ann Surg 256:476-486

13. Schochl H, Nienaber U, Hofer G et al (2010) Goal-directed coagulation management of major trauma patients using thromboelastometry (ROTEM)-guided administration of fibrinogen concentrate and prothrombin complex concentrate. Crit Care 14:R55

14. Tapia NM, Chang A, Norman M et al (2013) TEG-guided resuscitation is superior to standardized MTP resuscitation in massively transfused penetrating trauma patients. J Trauma Acute Care Surg 74:378-385

15. Einersen PM, Moore EE, Chapman MP et al (2017) Rapid-thrombelastography ( $r$-TEG) thresholds for goal-directed resuscitation of patients at risk for massive transfusion. J Trauma Acute Care Surg 82:114-119

16. Afshari A, Wikkelsoe A, Brok J, Moeller AM, Wetterslev J (2011) Thrombelastography (TEG) or thrombelastometry (ROTEM) to monitor haemotherapy versus usual care in patients with massive transfusion. Cochrane Database Syst Rev 16:CD007871. https://doi.org/10.1002/14651858. CD007871.pub2.

17. Da Luz LT, Nascimento B, Shankarakutty AK, Rizoli S, Adhikari NK (2014) Effect of thromboelastography (TEG $\left.{ }^{\circledR}\right)$ and rotational thromboelastometry $\left(\right.$ ROTEM $\left.^{\circledR}\right)$ on diagnosis of coagulopathy, transfusion guidance and mortality in trauma: descriptive systematic review. Crit Care 18:518

18. Hunt H, Stanworth S, Curry N, et al (2015) Thromboelastography (TEG) and rotational thromboelastometry (ROTEM) for trauma induced coagulopathy in adult trauma patients with bleeding (Review). Cochrane Database Syst Rev 16:CD 010438. https://doi.org/10.1002/14651858. CD010438.pub2

19. Veigas PV, Callum J, Rizoli S, Nascimento B, da Luz LT (2016) A systematic review on the rotational thrombelastometry $\left(\right.$ ROTEM $\left.^{\circledR}\right)$ values for the diagnosis of coagulopathy, prediction and guidance of blood transfusion and prediction of mortality in trauma patients. Scand J Trauma, Resusc Emerg Med 24:114 
20. Winearls J, Reade M, Miles H et al (2016) Targeted coagulation management in severe trauma: the controversies and the evidence. Anesth Analg 123:910-924

21. Baksaas-Aasen K, van Dieren S, Balvers K et al (2019) Data-driven development of ROTEM and TEG algorithms for the management of trauma hemorrhage. Ann Surg 270:1178-1185

22. Baksaas-Aasen K, Gall L, Eaglestone S et al (2017) iTACTIC_implementing treatment algorithms for the correction of trauma-induced coagulopathy: study protocol for a multicentre, randomised controlled trial. Trials 18:486

23. Gennarelli TA, Champion HR, Sacco WJ, Copes WS, Alves WM (1989) Mortality of patients with head injury and extracranial injury treated in trauma centres. J Trauma 29:1193-1201

24. Frith D, Gosling JC, Gaarder C et al (2010) Definition and drivers of acute traumatic coagulopathy: clinical and experimental investigations. J Thromb Haemost 8:1919-1925

25. Gonzalez E, Moore EE, Moore HB et al (2016) Goal-directed hemostatic resuscitation of trauma-induced coagulopathy: a pragmatic randomized clinical trial comparing a viscoelastic assay to conventional coagulation assays. Ann Surg 263:1051-1059

26. Maegele M, Schöchl H, Menovsky T et al (2017) Coagulopathy and haemorrhagic progression in traumatic brain injury: advances in mechanisms, diagnosis, and management. Lancet Neurol 16:630-647
27. The CRASH-3 trial collaborators (2019) Effects of tranexamic acid on death, disability, vascular occlusive events and other morbidities in patients with acute traumatic brain injury (CRASH-3): a randomised, placebo-controlled trial. Lancet 394:1713-1723

28. Prehospital Tranexamic Acid Use for Traumatic Brain Injury (TXA): https:// clinicaltrials.gov/ct2/show/NCT01990768.Accessed 18st of August2020.

29. Steyerberg EW, Wiegers E, Sewalt C et al (2019) Case-mix, care pathways, and outcomes in patients with traumatic brain injury in CENTER-TBI: a European prospective, multicentre, longitudinal, cohort study. Lancet Neurol 18:923-934

30. Hawryluk GWJ, Aguilera S, Buki A et al (2019) A management algorithm for patients with intracranial pressure monitoring: the Seattle International Severe Traumatic Brain Injury Consensus Conference (SIBICC). Intensive Care Med 45:1783-1794

31. Chesnut R, Aguilera S, Buki A et al (2020) A management algorithm for adult patients with both brain oxygen and intracranial pressure monitor ing: the Seattle International Severe Traumatic Brain Injury Consensus Conference (SIBICC). Intensive Care Med 46:919-929

32. Asehnoune K, Balogh Z, Citerio G et al (2017) The research agenda for trauma critical care. Intensive Care Med 43:1340-1351 


\section{Supplementary Materials}

Viscoelastic haemostatic assay augmented protocols for major trauma haemorrhage (ITACTIC): a randomized, controlled trial.

\section{Authors}

K. Baksaas-Aasen*1 MD, L. S. Gall*2 PhD, J. Stensballe 3 PhD, N.P. Juffermans4 Prof, N. Curry5 MD, M. Maegele6 Prof, A. Brooks7 MD, C. Rourke2 BSc, S. Gillespie2 PhD, J. Murphy8 MSc, R. Maroni8 MSc, P. Vulliamy2 PhD, H. H. Henriksen3 MD, K. Holst Pedersen3 MSc, K. M. Kolstadbraaten 1 MSN, M. R. Wirtz4 MD, D. J. B. Kleinveld4 MD, N. Schäfer6 PhD, S. Chinna7 MB ChB, R. A. Davenport2 PhD, P.A. Naess1 Prof, J.C. Goslings4 Prof, S. Eaglestone2 PhD, S. Stanworth5 Prof, P. I. Johansson3 Prof, C. Gaarder1 Prof, K. Brohi2 Prof. *These authors contributed equally to the study

1. Oslo University Hospital, Oslo, Norway and Faculty of Medicine, University of Oslo, Norway

2. Centre for Trauma Sciences, Queen Mary University of London, London, United Kingdom

3. Copenhagen University Hospital, Rigshospitalet, Copenhagen, Denmark

4. Amsterdam University Medical Centres, Amsterdam, The Netherlands

5. Oxford University Hospital NHS Trust, John Radcliffe Hospital, Oxford, United Kingdom

6. Cologne- Merheim Medical Centre, University of Witten/Herdecke, Cologne, Germany

7. Nottingham University Hospitals NHS Trust, Nottingham, United Kingdom

8. Centre for Cancer Prevention, Wolfson Institute of Preventive Medicine, Queen Mary University of London, London, United Kingdom

\section{Corresponding author:}

Karim Brohi, Centre for Trauma Sciences, Queen Mary University of London, 4 Newark Street, London, E1 2AT, United Kingdom.

E-mail: k.brohi@qmul.ac.uk 
Table S1 Interventions \& 24-hour transfusion requirements

\begin{tabular}{ccccccc} 
Subgroup & $\begin{array}{c}\text { \% receiving study } \\
\text { intervention }\end{array}$ & $\begin{array}{c}\text { Time to 1st study } \\
\text { intervention } \\
\text { (mins) }\end{array}$ & $\begin{array}{c}\text { RBCs } \\
\text { (units) }\end{array}$ & $\begin{array}{c}\text { FFP/Octaplasma } \\
\text { (units) }\end{array}$ & $\begin{array}{c}\text { Fibrinogen } \\
\text { equivalent } \\
\text { (g) }\end{array}$ & $\begin{array}{c}\text { Platelets } \\
\text { (pools) }\end{array}$ \\
\hline
\end{tabular}

\begin{tabular}{|c|c|c|c|c|c|c|}
\hline Baselin & & & & & & \\
\hline All - In & (n with haemostas & & & & & \\
\hline CCT & $62 / 170(36 \%)$ & $80(60-106)$ & $3(1-6)$ & $4(2-6)$ & $0(0-4)$ & $1(0-1)$ \\
\hline VHA & $120 / 178(67 \%)^{* *}$ & $61(48-85)^{*}$ & $3(1-6)$ & $4(1-6)$ & $4(0-4)^{*}$ & $1(0-2)^{* *}$ \\
\hline
\end{tabular}

Per protocol (n with haemostasis $=286$ )

\begin{tabular}{|c|c|c|c|c|c|c|}
\hline $\mathrm{CCT}$ & $59 / 148(40 \%)$ & $79(56-107)$ & $3(2-6)$ & $4(2-7)$ & $0(0-4)$ & $1(0-2)$ \\
\hline VHA & $108 / 138(78 \%)^{* *}$ & $64(46-85)^{*}$ & $4(1-6)$ & $4(2-7)$ & $4(0-4)^{*}$ & $2(1-2)^{* *}$ \\
\hline
\end{tabular}

Severe TBI $(\mathrm{n}$ with haemostasis $=63)$

$\begin{array}{lcccccc}\text { CCT } & 6 / 26(23 \%) & 61(34-101) & 2(0-3) & 3(1-6) & 0(0-0) & 0(0-1) \\ \text { VHA } & 26 / 37(70 \%)^{* *} & 53(45-85) & 3(1-6)^{*} & 3(1-6) & 4(0-4)^{*} & 2(0-2)^{*}\end{array}$

No/Mild TBI (n with haemostasis = 281)

$\begin{array}{lrrrrrr}\text { CCT } & 56 / 142(40 \%) & 80(62-109) & 3(2-6) & 4(2-7) & 0(0-4) & 1(0-1) \\ \text { VHA } & 93 / 139(67 \%)^{* *} & 64(50-85)^{*} & 3(1-6) & 4(1-6) & 2(0-4) & 1(0-2)^{*}\end{array}$

\section{Injury to 24 hours}

All - Intention-to-treat $(n=396)$

$\begin{array}{lrrrrrr}\text { CCT } & 86 / 195(44 \%) & 187(143-234) & 6(4-10) & 7(4-11) & 3(0-4) & 1(0-2) \\ \text { VHA } & 139 / 201(69 \%)^{* *} & 164(130-194)^{*} & 6(3-10) & 6(3-10)^{*} & 4(0-4) & 2(1-3)^{*}\end{array}$

Per protocol $(n=313)$

$\begin{array}{lcccccc}\text { CCT } & 79 / 163(48 \%) & 189(143-232) & 6(4-11) & 8(4-12) & 4(0-4) & 1(0-2) \\ \text { VHA } & 122 / 150(81 \%)^{* *} & 164(127-195)^{*} & 6(4-10) & 6(4-10) & 4(0-8)^{*} & 2(1-3)^{*}\end{array}$

Severe TBI $(\mathbf{n}=\mathbf{7 4})$

$\begin{array}{lcccccc}\text { CCT } & 12 / 35(34 \%) & 153(111-222) & 6(4-10) & 6(2-9) & 0(0-4) & 1(0-2) \\ \text { VHA } & 29 / 39(74 \%)^{* *} & 144(118-197) & 6(4-8) & 4(4-8) & 4(0-4) & 2(1-3)^{*} \\ \text { No/Mild TBI }(\mathbf{n}=\mathbf{3 1 3}) & & & & & & \\ \text { CCT } & 73 / 156(47 \%) & 191(152-233) & 6(4-10) & 8(4-12) & 4(0-4) & 1(0-2) \\ \text { VHA } & 108 / 157(69 \%)^{* *} & 164(133-190)^{*} & 6(3-10) & 6(3-10)^{*} & 4(0-5) & 2(1-3)\end{array}$

The results are given as median (interquartile range), except for $\%$ receiving study intervention. Data for Baseline to Haemostasis does not include patients who died before haemostasis. Transfusion components are the total number of components given, both empiric and study directed. The statistical comparisons in this table are all post-hoc analyses, except for those for the RBCs, FFP/Octaplasma and platelets in the ITT cohort. A correction for multiple testing has not been applied. 
A small number of patients in each subgroup did not have a record for all measures (missing numbers not shown). In the ITT cohort, the number of missing records reached $9 \%$ for some of the measures.

$\wedge$ Results given as number/number in subgroup (percentage).

* p-value for the difference between the two groups of less than 0.05

** p-value for the difference between the two groups of less than 0.001

RBC Red Blood Cell, FFP Fresh Frozen Plasma, CCT Conventional Coagulation Test, VHA Viscoelastic Haemostatic Assay, PTr Prothrombin Time ratio, TBI Traumatic Brain Injury 


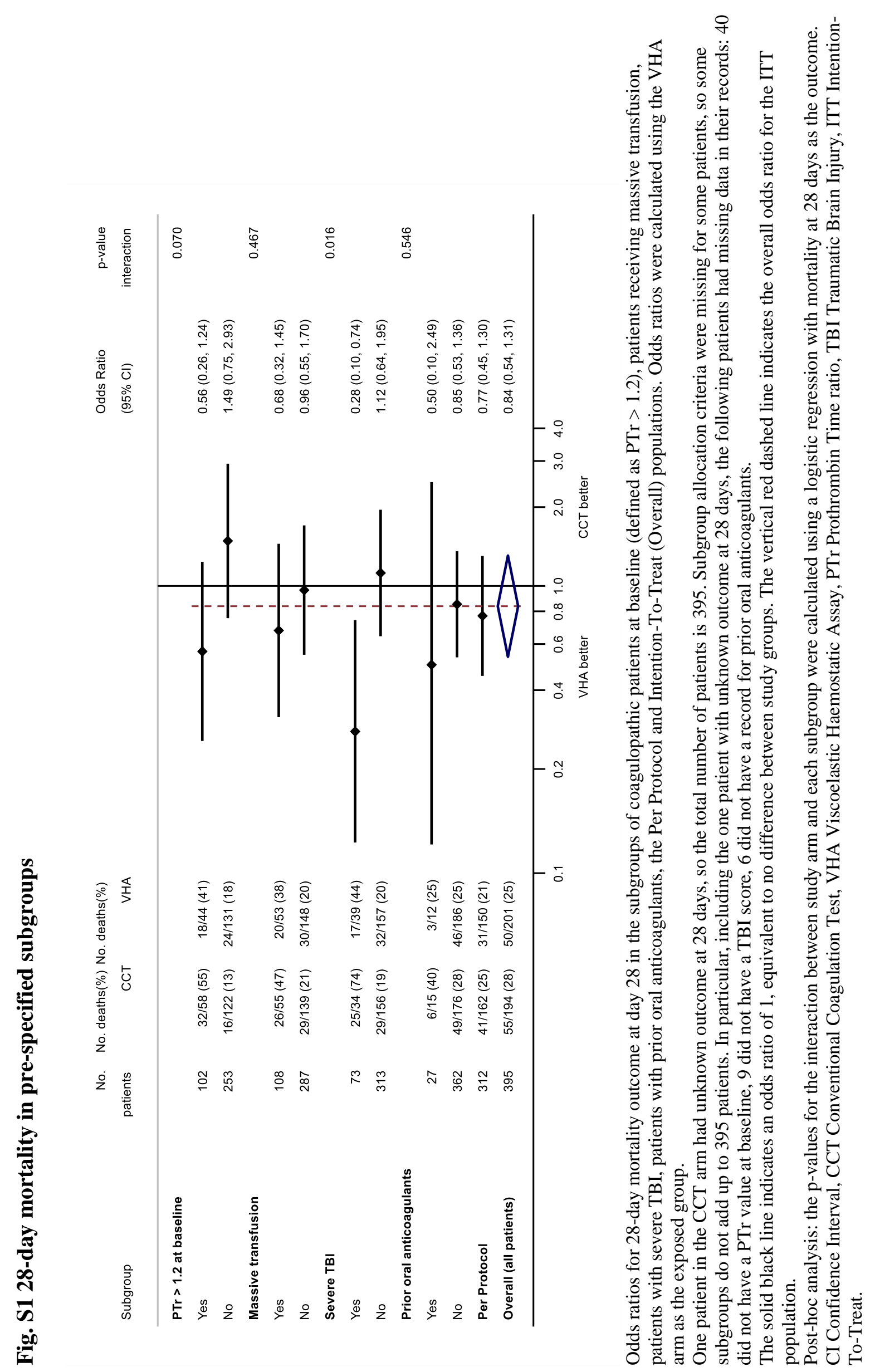


Table S2 Admission characteristics in severe TBI predefined subgroup (intention-totreat)

\begin{tabular}{lcc} 
& $\begin{array}{c}\text { CCT } \\
(\mathbf{n}=\mathbf{3 5})\end{array}$ & $\begin{array}{c}\text { VHA } \\
(\mathbf{n}=\mathbf{3 9})\end{array}$ \\
\hline Median age $(\mathrm{IQR})_{+}$ & $39(26-54)$, & $40(24-56)$, \\
& $\mathrm{n}=35$ & $\mathrm{n}=39$ \\
Male sex - no. $(\%)_{+}$ & $29 / 35(83 \%)$ & $22 / 39(56 \%)$
\end{tabular}

\section{Injuries \& Admission Physiology}

Median Injury Severity Score (IQR)

Injury caused by blunt trauma alone - no. (\%)

$\begin{array}{cc}38(29-43), & 41(34-54), \\ \mathrm{n}=35 & \mathrm{n}=39 \\ 25 / 35(71 \%) & 30 / 39(77 \%) \\ & \\ 90(75-110), & 112(87-128), \\ \mathrm{n}=31 & \mathrm{n}=37 \\ 87(58-100), & 80(60-120), \\ \mathrm{n}=28 & \mathrm{n}=35 \\ 3(3-3), & 3(3-6), \\ \mathrm{n}=34 & \mathrm{n}=39 \\ 8.7(4.0-13.9), & 10.1(7.9-16.3), \\ \mathrm{n}=34 & \mathrm{n}=36 \\ 5.2(3.0-10.5), & 5.3(3.3-8.7), \\ \mathrm{n}=34 & \mathrm{n}=38\end{array}$

Admission Coagulation Profile

Patients with PTr $>1.2$ - no. (\%)

$\begin{array}{cc}15 / 32(47 \%) & 16 / 35(46 \%) \\ 1.4(1.1-2.1), & 1.5(0.9-2.1), \\ \mathrm{n}=30 & \mathrm{n}=34\end{array}$

Median fibrinogen (IQR) - $\mathrm{g} / \mathrm{L}$

\section{Pre-baseline therapy}

Received tranexamic acid bolus - no. (\%)

$33 / 35(94 \%) \quad 35 / 39(90 \%)$

Median units of RBCs (IQR)

$\begin{array}{cc}3(1-4), & 2(1-4), \\ \mathrm{n}=31 & \mathrm{n}=37 \\ 0(0-3), & 0(0-2), \\ \mathrm{n}=31 & \mathrm{n}=37 \\ 0(0-0), & 0(0-0), \\ \mathrm{n}=32 & \mathrm{n}=35 \\ 0(0-0), & 0(0-0), \\ \mathrm{n}=31 & \mathrm{n}=37\end{array}$

TBI Traumatic Brain Injury, CCT Conventional Coagulation Test, VHA Viscoelastic Haemostatic Assay, IQR Interquartile Range, AIS Abbreviated Injury Scale, RBC Red Blood Cells, FFP Fresh Frozen Plasma. PTr Prothrombin Time ratio. 
Table S3 Adjusted analysis of the severe TBI subgroup

\begin{tabular}{|c|c|c|}
\hline & $\begin{array}{l}\text { Alive and Free of Massive } \\
\text { Transfusion at } 24 \text { Hours } \\
\text { (Primary Endpoint) }\end{array}$ & 28-day Mortality \\
\hline Un-Adjusted & $\mathrm{OR}=2.12(95 \% \mathrm{CI} 0.83-5.39)$ & $\mathrm{OR}=0.28(95 \%$ CI $0.10-0.74)$ \\
\hline \multicolumn{3}{|l|}{ Univariate Adjusted } \\
\hline Age & $\mathrm{OR}=2.53(95 \%$ CI $0.91-7.09)$ & $\mathrm{OR}=0.28(95 \%$ CI $0.10-0.74)$ \\
\hline Sex & $\mathrm{OR}=1.92(95 \%$ CI $0.73-5.06)$ & $\mathrm{OR}=0.30(95 \% \mathrm{CI} 0.11-0.82)$ \\
\hline Type of Injury (blunt/penetrating) & $\mathrm{OR}=2.26(95 \% \mathrm{CI} 0.87-5.88)$ & $\mathrm{OR}=0.25(95 \%$ CI $0.09-0.69)$ \\
\hline Heart Rate at admission & $\mathrm{OR}=1.59(95 \%$ CI $0.57-4.42)$ & $\mathrm{OR}=0.33(95 \%$ CI $0.11-0.97)$ \\
\hline $\begin{array}{l}\text { Systolic Blood Pressure at } \\
\text { admission }\end{array}$ & $\mathrm{OR}=1.53(95 \% \mathrm{CI} 0.54-4.31)$ & $\mathrm{OR}=0.26(95 \%$ CI $0.08-0.81)$ \\
\hline $\begin{array}{l}\text { Glasgow Coma Scale score at } \\
\text { admission }\end{array}$ & $\mathrm{OR}=2.00(95 \% \mathrm{CI} 0.78-5.12)$ & $\mathrm{OR}=0.21(95 \% \mathrm{CI} 0.07-0.64)$ \\
\hline Base Deficit at admission & $\mathrm{OR}=2.74(95 \%$ CI $0.96-7.77)$ & $\mathrm{OR}=0.21(95 \%$ CI $0.06-0.68)$ \\
\hline $\begin{array}{l}\text { Multivariate Adjusted } \\
\text { (all of the covariates above) }\end{array}$ & $\mathrm{OR}=2.10(95 \% \mathrm{CI} 0.51-8.61)$ & $\mathrm{OR}=0.16(95 \%$ CI $0.03-0.90)$ \\
\hline
\end{tabular}

Odds ratios for the primary outcome (alive and free of massive transfusion at 24 hours) and 28-day mortality in the subgroup of patients with severe TBI, risk adjusted for admission characteristics. Odds ratios were calculated using the VHA arm as the exposed group. 